J. DIFFERENTIAL GEOMETRY

59 (2001) $87-176$

\title{
A PROOF OF THE FINITE FILLING CONJECTURE
}

\author{
STEVEN BOYER \& XINGRU ZHANG
}

\begin{abstract}
Let $M$ be a compact, connected, orientable, hyperbolic 3-manifold whose boundary is a torus. We show that there are at most five slopes on $\partial M$ whose associated Dehn fillings have either a finite or an infinite cyclic fundamental group. Furthermore, the distance between two slopes yielding such manifolds is no more than three, and there is at most one pair of slopes which realize the distance three. Each of these bounds is realized when $M$ is taken to be the exterior of the figure- 8 sister knot.
\end{abstract}

\section{Introduction}

Let $M$ be a compact connected orientable 3-manifold whose boundary is a torus. We call such 3 -manifold a knot exterior. We shall assume throughout this paper that knot exteriors are hyperbolic, that is, their interiors admit a complete hyperbolic metric of finite volume. A slope $r$ on $\partial M$ is the $\partial M$-isotopy class of an unoriented essential simple closed curve it contains. The set of slopes on $\partial M$ can be identified with the \pm pairs of primitive homology classes in $H_{1}(\partial M)$ - a slope $r$ determines a primitive homology class $H_{1}(\partial M)$, well-defined up to sign, obtained by orienting a representative curve for $r$ and considering the homology class it carries. As usual, we use $\Delta\left(r_{1}, r_{2}\right)$ to denote the distance between two slopes $r_{1}$ and $r_{2}$ on $\partial M$, i.e., their minimal geometric intersection number on $\partial M$. The distance between two slopes coincides with the absolute value of the algebraic intersection number between the corresponding homology classes.

Received 05/22/01.

S. Boyer was partially supported by grants: NSERC OGP 0009446 and FCAR EQ 3518.

X. Zhang was partially supported by NSERC grant OGP 0170284 . 
The Dehn filling of $M$ with slope $r$ is the manifold $M(r)$ obtained by attaching a solid torus $V$ to $M$ by a homeomorphism of $\partial V \rightarrow \partial M$ which sends a meridian curve of $V$ to a simple closed curve in $\partial M$ of the given slope $r$. W. Thurston has shown [34] that all but finitely many fillings of $M$ are hyperbolic manifolds and a fundamental problem is to determine constraints on the set of slopes $r$ on $\partial M$ for which $M(r)$ is not hyperbolic. This occurs, for instance, if $\pi_{1}(M(r))$ is either a finite or an infinite cyclic group. The Cyclic Filling Theorem, due to M. Culler, C. McA. Gordon, J. Luecke and P. Shalen [11], provides the model for the type of result which can be expected. If $\mathcal{C}$ denotes the set of slopes $r$ on $\partial M$ such that $\pi_{1}(M(r))$ is cyclic, then it states that $\mathcal{C}$ contains no more than three slopes and the distance between any two slopes in $\mathcal{C}$ is at most 1 . One can visualize this result as follows: the cyclic filling theorem is equivalent to the statement that there is a basis $\alpha, \beta$ of $H_{1}(\partial M)$ such that the pairs of classes corresponding to $\mathcal{C}$ are contained in $\pm\{\alpha, \beta, \alpha+\beta\}$.

Next consider the set $\mathcal{F}$ of slopes $r$ on $\partial M$ such that $\pi_{1}(M(r))$ is either finite or infinite cyclic. In his address to the 1990 ICM in Kyoto [16], Cameron Gordon conjectured that the distance between any two slopes in $\mathcal{F}$ is no more than 3. Since that time his conjecture has taken on the more definitive form below (see Conjecture B of Gordon's problem 1.77 in [21]).

The Finite Filling Conjecture [C. McA. Gordon]. For a hyperbolic knot exterior $M$, there are at most 5 finite or infinite cyclic filling slopes on $\partial M$ and the distance between any two such slopes is at most 3. Further the distance 3 is realized by at most one pair of slopes.

The number 5 and the distance 3 in the statement of the finite filling conjecture are the best upper bounds that one can expect they are realized on an example due to Jeff Weeks (see Example 11.7). An elementary argument shows that the conjecture is equivalent to the statement that there is a basis $\alpha, \beta$ of $H_{1}(\partial M)$ such that \pm -pairs of primitive classes in $H_{1}(\partial M)$ corresponding to $\mathcal{F}$ are contained in $\pm\{\alpha, \beta, \alpha+\beta, \alpha+2 \beta, \alpha+3 \beta\}$.

Let $\# \mathcal{F}$ denote the number of slopes in $\mathcal{F}$ and $\Delta(\mathcal{F})$ the maximum distance between a pair of its slopes. Shortly after Gordon announced his conjecture, S. Bleiler and C. Hodgson obtained the inequalities $\# \mathcal{F} \leq 24$ and $\Delta(\mathcal{F}) \leq 23$ [1] through an analysis of when the manifolds $M(r)$ admit a Riemannian metric of strictly negative sectional curvature. More recently, S. Boyer and X. Zhang obtained the 
bounds $\# \mathcal{F} \leq 6$ and $\Delta(\mathcal{F}) \leq 5$ in work which should be thought of as a continuation of [11]. This line of thought is further developed here, leading to a proof of the conjecture.

Theorem 1.1. The Finite Filling Conjecture is true.

Of particular significance is the case where $M$ is the exterior $M_{K}$ of a hyperbolic knot $K$ in the 3 -sphere. Let $\mu_{K}$ denote the meridinal slope of $K$. This is the slope on $\partial M$ represented by an essential curve which bounds a disk in a tubular neighbourhood of $K$. It is conjectured (see Conjecture A in problem 1.77 [21]) that if $r$ is a slope on $\partial M_{K}$ for which $\pi_{1}\left(M_{K}(r)\right)$ is a finite group, then $\Delta\left(r, \mu_{K}\right) \leq 1$.

Theorem 1.2. $\quad$ Let $K$ be a hyperbolic knot in $S^{3}$ and let $M_{K}$ denote its exterior. There is at most one finite filling slope $r$ on $\partial M_{K}$ satisfying $\Delta\left(r, \mu_{K}\right) \geq 2$, and if there is one, $\Delta\left(r, \mu_{K}\right)=2$.

Proof. It was proved in [2] that $\Delta\left(r, \mu_{K}\right) \leq 2$ for any finite filling slope $r$. Since the distance between any two slopes $r_{1} \neq r_{2}$ on $\partial M_{K}$ for which $\Delta\left(r_{j}, \mu_{K}\right)=2$ is divisible by 4 , Theorem 1.1 implies that not both of $r_{1}, r_{2}$ can be finite filling slopes. $\quad$ q.e.d.

Specializing further, suppose that $M_{K}$ is the exterior of an amphicheiral hyperbolic knot $K$ in the 3 -sphere. If $\mu, \lambda \in H_{1}\left(\partial M_{K}\right)$ are the primitive classes corresponding to a meridian-longitude pair for $K$, then the slopes on $\partial M_{K}$ are in bijective correspondence with $\mathbb{Q} \cup\left\{\frac{1}{0}\right\}$ via "slope $r \leftrightarrow \pm(p \mu+q \lambda) \leftrightarrow \frac{p}{q}$ ". We remind the reader that $H_{1}\left(M_{K}\left(\frac{p}{q}\right)\right) \cong \mathbb{Z}_{p}$.

Theorem 1.3. If $K \subset S^{3}$ is an amphicheiral hyperbolic knot, then the only fillings, other than the trivial one, which can possibly yield a manifold with a finite fundamental group, are those corresponding to 1 and -1 . In particular, only the binary icosahedral group can occur as the fundamental group of a manifold with a finite fundamental group obtained by a nontrivial filling of an amphicheiral knot exterior.

Proof. Note that as $K$ is amphicheiral, $M_{K}\left(\frac{p}{q}\right)$ is homeomorphic to $M_{K}\left(\frac{-p}{q}\right)$ for all slopes $\frac{p}{q} \in \mathbb{Q} \cup\left\{\frac{1}{0}\right\}$. Hence if $\frac{p}{q}$ is a nontrivial finite filling slope of $M_{K}$, then $\frac{-p}{q}$ is as well, yielding a manifold with the same fundamental group. Note that $q \neq 0$ as the slope is nontrivial, while $p \neq 0$ because otherwise, $H_{1}\left(M_{K}\left(\frac{p}{q}\right)\right)$ would be infinite. Thus $|p q| \geq 1$. On the other hand, the finite filling conjecture implies that $3 \geq$ 
$\Delta\left(\frac{p}{q}, \frac{-p}{q}\right)=2|p q|$ and therefore $|p q| \leq 1$. Hence $\frac{p}{q}= \pm 1$. Consideration of the abelianizations of the seven types of finite groups which can be isomorphic to the fundamental group of a 3-manifold ( $\$ 2)$ shows that the fundamental group of $M( \pm 1)$ is either the trivial group or the binary icosahedral group. But since $\Delta(1,-1)=2$, the cyclic filling theorem implies that the first possibility cannot arise. The proof of the theorem is therefore complete.

q.e.d.

We will sketch our proof of the finite filling conjecture in what remains of the introduction, and then describe the organization of the paper.

Let $\pi$ denote the fundamental group of $M$. The set of characters of representations of $\pi$ with values in $S L(2, \mathbb{C})$ may be identified with the points of a complex affine algebraic set $X(M)$ [12]. Since $M$ is hyperbolic, $X(M)$ contains the character $\chi$ of a discrete faithful representation of $\pi$ in $S L(2, \mathbb{C})$. It turns out that any algebraic component $X_{0}$ of $X(M)$ containing $\chi$ is a curve [13] and Culler and Shalen have shown how such a curve determines a Culler-Shalen norm $\|\cdot\|: H_{1}(\partial M ; \mathbb{R}) \rightarrow$ $[0, \infty)$ (see Chapter 1 of [11]). Roughly speaking, if $r$ is the slope associated to a primitive element $\alpha \in H_{1}(\partial M)$, then $\|\alpha\|$ measures the number of characters of representations $\pi_{1}(M(r)) \rightarrow S L(2, \mathbb{C})$.

The unit $\|\cdot\|$-ball $B$ is a finited-sided, convex, balanced polygon which encodes topological information about $M$ in a striking way - the vertices of $B$ are rational multiples of primitive elements in $H_{1}(\partial M)$ whose associated slopes are the slopes of the boundaries of certain essential surfaces in $M$. It turns out that amongst all the nontrivial classes in $H_{1}(\partial M ; \mathbb{Z})$, the norm $\|\cdot\|$ takes on relatively small values on those classes which correspond to finite or cyclic filling slopes, but which are not boundary slopes ([11] and [2]). This suggests that not only are there few such classes, but that they are "close" to each other. In fact this idea was one of the essential ingredients used in [2] to deduce the inequalities $\# \mathcal{F} \leq 6$ and $\Delta(\mathcal{F}) \leq 5$. More can be said though. It follows from [2] and [4] that the conjecture holds except perhaps for a finite number of explicitly given norms. The new element we introduce in this paper to deal with the remaining open cases is the A-polynomial, due to D. Cooper, M. Culler, H. Gillet, D. Long and P. Shalen [8]. We describe it now.

Fix a basis $\mu, \lambda$ of $\pi_{1}(\partial M) \cong \mathbb{Z}^{2}$ and let $D_{0}$ be the closure in $\mathbb{C}^{2}$ of the set of all pairs $(u, v)$ where there is some representation $\rho: \pi \rightarrow S L(2, \mathbb{C})$ satisfying: 
- $\chi_{\rho} \in X_{0}$.

- $\rho \mid \pi_{1}(\partial M)$ is upper-triangular.

- $\rho(\mu)=\left(\begin{array}{cc}u & * \\ 0 & u^{-1}\end{array}\right), \rho(\lambda)=\left(\begin{array}{cc}v & * \\ 0 & v^{-1}\end{array}\right)$.

It turns out that $D_{0}$ is a plane algebraic curve and is thus defined by a polynomial, called the $A$-polynomial of $X_{0}$. In order to exploit this construction to our best advantage, it is essential for us to work with a polynomial having integer coefficients. One way to achieve this is to replace $X_{0}$ by its orbit $C_{\mathrm{M}}$ under the natural Aut $(\mathbb{C}) \times H^{1}\left(M ; \mathbb{Z}_{2}\right)$ action on the components of $X(M)(\S 5)$. The curve $C_{\mathrm{M}}$ has various useful properties, but in particular the plane curve $D_{\mathrm{M}}$ it determines (as above) is defined over the rationals. Hence it is the zero set of a primitive polynomial $p \in \mathbb{Z}[u, v]$ without repeated factors. We take the $A$-polynomial of $C_{\mathrm{M}}$, written

$$
A_{\mathrm{M}}(u, v)=\sum_{(m, n)} b_{m, n} u^{m} v^{n} \in \mathbb{Z}[u, v]
$$

to be a certain power of $p(\S 6)$, the power being chosen to better reflect the close relationship between $A_{\mathrm{M}}$ and the Culler-Shalen norm $\|\cdot\|_{\mathrm{M}}$ determined by $C_{\mathrm{M}}$. This definition is a natural consequence of the applications we have in mind, but we warn the reader that following the original definitions ([8]), the $A$-polynomial of $C_{\mathrm{M}}$ would have been taken to be $p(u, v)$.

The Newton polygon $N_{\mathrm{M}}$ of $A_{\mathrm{M}}$ is the convex hull of $\left\{(m, n) \mid b_{m, n} \neq\right.$ $0\}$. The notion of width, due to P. Shanahan [31], is introduced in $\S 7$ and is used to show how the geometry of $N_{\mathrm{M}}$ determines the Culler-Shalen norm $\|\cdot\|_{\mathrm{M}}$ of $C_{\mathrm{M}}(\S 8)$. One consequence of this relationship is that $N_{\mathrm{M}}$ and $B_{\mathrm{M}}$, the $\|\cdot\|_{\mathrm{M}}$-ball of radius $s_{\mathrm{M}}=\min \left\{\|\alpha\|_{\mathrm{M}} \mid \alpha \in H_{1}(\partial M) \backslash\{0\}\right\}$ determine each other in a very nice way.

Theorem 1.4. The Newton polygon $N_{M}$ is dual to $B_{M}$ in the following sense. The line through any pair of antipodal vertices of $B_{M}$ is parallel to a side of $N_{M}$. Conversely the line through any pair of antipodal vertices of $N_{M}$ is parallel to a side of $B_{M}$.

This relation gives, in particular, a different and simple proof of one of the main results of [8] — the slope of a side of the Newton polygon is a boundary slope of $M$ (see Corollary 8.4). 
Our proof of the finite filling conjecture can now be described. Consider one of the putative Culler-Shalen norms where the work in [2] and [4] does not suffice to prove the conjecture, and suppose that $M$ is a hyperbolic knot exterior for which $\|\cdot\|_{M}$ coincides with this norm. From the preceding discussion, we can determine precisely what the polygon $N_{\mathrm{M}}$ would have to be. Now the coefficients of $A_{\mathrm{M}}$ are constrained in various ways. For instance Cooper and Long have shown that $b_{m, n}= \pm 1$ if $(m, n)$ is a corner of $N_{\mathrm{M}}$ [10]. Further it follows from [8] that the edge polynomials of $A_{\mathrm{M}}$ (see $\S 6$ ) are products of cyclotomic polynomials. We show in $\S 11$ that the assumption that a filling of $M$ along a given slope yields a manifold with a finite fundamental group implies that the roots of an associated specialization of the variables in the $A$-polynomial are either \pm 1 or certain roots of unity. It turns out that in each of the cases we consider, except one, we are able to use these constraints to show that the conjecture holds. In this one bad case, the constraints allow us to determine $A_{\mathrm{M}}$, but do not lead to a contradiction. Nevertheless, in the appendix we are able to prove that this polynomial is not the $A$-polynomial of any hyperbolic knot exterior $M$. The idea behind our argument goes back to [8]. There it is described how work of C. Hodgson shows that if the given polynomial was the $A$-polynomial of a hyperbolic 3 -manifold $M$, then the real 1 -form

$$
\omega=\ln |u| d(\arg (v))-\ln |v| d(\arg (u))
$$

is exact on the smooth part of $D_{\mathrm{M}}=A_{\mathrm{M}}^{-1}(0)$. In particular the integral of $\omega$ over any closed, piecewise-smooth loop in $D_{\mathrm{M}}$ is zero. We find an explicit closed curve in $D_{\mathrm{M}}$ on which this condition fails. Arguments of this type were first used by D. Cooper and D. Long in $[9, \S 10]$. We are grateful to Daryl Cooper for his suggestions concerning our calculations and for verifying them with his own computer programme.

The paper is organized as follows. In $\S 2,3,4$ some of the work of $[2]$ is recalled, refined and further developed. The action of $\operatorname{Aut}(\mathbb{C}) \times$ $H^{1}\left(M ; \mathbb{Z}_{2}\right)$ on the components of the character variety $X(M)$ is discussed in $\S 5$. The theory of $A$-polynomials is broached in $\S 6$ and that of width in $\S 7$ with an eye to deriving various relationships between $A$-polynomials and Culler-Shalen norms in $\S 8$. We specialize these constructions to the canonical curve $C_{\mathrm{M}}$ and develop its particular properties in $\S 9$ and $\S 10$. Applications to Dehn filling is the purpose of $\S 11$. Our proof of the finite filling conjecture is outlined in $\S 12$, where we split it into five propositions which are examined successively over the paper's next five sections. In the last section we make some general 
remarks on finite surgery on knots in the 3 -sphere and give a new proof that hyperbolic 2-bridge knots admit no nontrivial finite surgeries (due to Delman [14] and independently to Tanguay [32]). Finally, there is an appendix which provides a proof that a certain polynomial in $\mathbb{Z}[u, v]$ cannot be the $A$-polynomial of a hyperbolic knot exterior.

\section{Preliminaries}

Our references for the basic notions, terminology and notation relating to the topology of 3-manifolds are [19] and [20], for knot theory [29], for algebraic geometry [30], and for the $S L(2, \mathbb{C})$-character varieties of 3-manifolds [12].

All manifolds are assumed to be orientable and smooth, unless otherwise specified. By a surface we mean a compact 2-manifold. By an essential surface in a compact 3-manifold we mean a properly embedded, incompressible surface no component of which is $\partial$-parallel and no 2-sphere component of which bounds a 3-ball. A 3-manifold is called irreducible if it does not contain an essential 2-sphere, and reducible otherwise.

Throughout the paper, $M$ will denote a hyperbolic knot exterior. A slope $r$ on $\partial M$ is called a boundary slope if there is an essential surface $F$ in $M$ such that $\partial F \cap \partial M$ is a nonempty set of parallel simple closed curves on $\partial M$ of slope $r$. A boundary slope $r$ on $\partial M$ is said to be strict if there is an essential surface $F$ in $M$ such that $F$ is not the fiber in any representation of $M$ as a fiber bundle over the circle and such that $\partial F \cap \partial M$ is a nonempty set of parallel simple closed curves on $\partial M$ of slope $r$.

The finite groups which can arise as the fundamental groups of closed, orientable 3-manifolds are contained among the seven following families [25]:

C-type: Cyclic groups $\mathbb{Z}_{j}=\mathbb{Z} / j \mathbb{Z}$ for $j \geq 1$.

Even D-type: $D_{4 n} \times \mathbb{Z}_{j}$ with $n \geq 2$ even, $j \geq 1$ and $\operatorname{gcd}(n, j)=1$, where $D_{4 n}=\left\langle x, y \mid x^{2}=(x y)^{2}=y^{n}\right\rangle$ is the binary dihedral group of order $4 n$.

Odd D-type: $D\left(2^{k}, 2 l+1\right) \times \mathbb{Z}_{j}$ for $k \geq 2, j \geq 1, l \geq 1, \operatorname{gcd}(2(2 l+$ $1), j)=1$, where $D\left(2^{k}, 2 l+1\right)=\langle x, y| x^{2^{k}}=1, y^{2 l+1}=1, x y x^{-1}=$ $\left.y^{-1}\right\rangle$. Note that $D\left(2^{2}, 2 l+1\right)$ is isomorphic to the binary dihedral group $D_{4(2 l+1)}=\left\langle x, y \mid x^{2}=(x y)^{2}=y^{2 l+1}\right\rangle$ of order $4(2 l+1)$. 
T-type: $T\left(8,3^{k}\right) \times \mathbb{Z}_{j}$ for $k \geq 1, j \geq 1, \operatorname{gcd}(6, j)=1$, where $T\left(8,3^{k}\right)=\left\langle x, y, z \mid x^{2}=(x y)^{2}=y^{2}, z^{3^{k}}=1, z x z^{-1}=y, z y z^{-1}=x y\right\rangle$. Note that $T(8,3)$ is isomorphic to the binary tetrahedral group $T_{24}=$ $\left\langle x, y \mid x^{2}=(x y)^{3}=y^{3}, x^{4}=1\right\rangle$.

O-type: $O_{48} \times \mathbb{Z}_{j}$ for $j \geq 1, \operatorname{gcd}(6, j)=1$, where $O_{48}=\langle x, y| x^{2}=$ $\left.(x y)^{3}=y^{4}, x^{4}=1\right\rangle$ is the binary octahedral group.

I-type: $I_{120} \times \mathbb{Z}_{j}$ for $j \geq 1, \operatorname{gcd}(30, j)=1$, where $I_{120}=\langle x, y| x^{2}=$ $\left.(x y)^{3}=y^{5}, x^{4}=1\right\rangle$ is the binary icosahedral group.

Q-type: $Q(8 n, k, l) \times \mathbb{Z}_{j}$, where $Q(8 n, k, l)=\langle x, y, z| x^{2}=(x y)^{2}=$ $\left.y^{2 n}, z^{k l}=1, x z x^{-1}=z^{r}, y z y^{-1}=z^{-1}\right\rangle, n, k, l, j$ are relatively prime odd positive integers, $r \equiv-1(\bmod k)$ and $r \equiv 1(\bmod l)$.

We call a slope $r$ on $\partial M$ a finite filling slope or a cyclic filling slope if $M(r)$ has, respectively, a finite or a cyclic fundamental group. If $r$ is a slope on $\partial M$ such that the fundamental group of $M(r)$ is of one of the types listed above, then we shall say that $r$ is a finite filling slope of that type.

Lemma 2.1. If $M$ admits a finite filling slope $r$, then $H_{1}(M ; \mathbb{Q}) \cong$ $\mathbb{Q}$. Further:

(1) If $r$ is a T-type or I-type slope, then $H_{1}\left(M(r) ; \mathbb{Z}_{2}\right) \cong 0$ and $H_{1}\left(M ; \mathbb{Z}_{2}\right) \cong \mathbb{Z}_{2}$.

(2) If $r$ is an O-type or odd D-type slope, then $H_{1}\left(M(r) ; \mathbb{Z}_{2}\right) \cong \mathbb{Z}_{2}$ and $H_{1}\left(M ; \mathbb{Z}_{2}\right) \cong \mathbb{Z}_{2}$ or $\mathbb{Z}_{2} \oplus \mathbb{Z}_{2}$.

(3) If $r$ is an even D-type or $Q$-type, then $H_{1}\left(M(r) ; \mathbb{Z}_{2}\right) \cong \mathbb{Z}_{2} \oplus \mathbb{Z}_{2}$.

Proof. The proof is a simple homological argument which can be easily deduced from [2, Lemma 5.1].

q.e.d.

Proposition 2.2 ([11]). If $r$ is a finite or cyclic filling slope in $\partial M$ and is also a boundary slope, then $\Delta\left(r, r^{\prime}\right) \leq 1$ for any other finite or cyclic filling slope $r^{\prime}$.

Proof. If the first Betti number of $M$ is 1, then the conclusion of the lemma follows from [11, Theorem 2.0.3]. If the first Betti number of $M$ is larger than 1, then by Lemma 2.1, both $r$ and $r^{\prime}$ must be (infinite) cyclic filling slopes. Thus $\Delta\left(r, r^{\prime}\right) \leq 1$ by the cyclic surgery theorem of [11]. 
We shall frequently use $V$ and $L$ to denote $H_{1}(\partial M ; \mathbb{R})$ and $H_{1}(\partial M$; $\mathbb{Z})$ respectively. Once we have fixed an ordered basis $\{\mu, \lambda\}$ of $L$, we shall often identify the pair $(V, L)$ with $\left(\mathbb{R}^{2}, \mathbb{Z}^{2}\right)$ by associating $\mu$ to $(1,0)$ and $\lambda$ to $(0,1)$.

By a pair of elements in $V$ we mean a \pm pair $\{(a, b),(-a,-b)\}$. A slope on $\partial M$ determines, and is determined by, a pair of primitive elements of $L$. We call a primitive homology class a boundary class, or a strict boundary class, or a cyclic filling class or a finite filling class, etc., if the corresponding slope has that property.

For a primitive class $\alpha \in L$ corresponding to a slope $r$ on $\partial M$, the manifold $M(r)$ will also be denoted by $M(\alpha)$.

If two slopes $r_{1}$ and $r_{2}$ correspond to $\pm\left(p_{1}, q_{1}\right), \pm\left(p_{2}, q_{2}\right) \in \mathbb{Z}^{2}$, then basic surface topology can be used to show that $\Delta\left(r_{1}, r_{2}\right)$ coincides with the absolute value of the algebraic intersection number between the classes in $H_{1}(\partial M)$ corresponding to $\left(p_{1}, q_{1}\right)$ and $\left(p_{2}, q_{2}\right)$. Thus

$$
\Delta\left(r_{1}, r_{2}\right)=\left|p_{1} q_{2}-p_{2} q_{1}\right| .
$$

Consequently, for any two elements $\alpha$ and $\beta$ in $H_{1}(\partial M ; \mathbb{Z})$, we use $\Delta(\alpha, \beta)$ to denote the absolute value of their algebraic intersection number.

Any rank 2 subgroup of the homology group $L$ will be referred to as a sublattice.

The following lemma will prove useful later in the paper.

Lemma 2.3. Let $\widetilde{L}$ be a sublattice of $L=H_{1}(\partial M ; \mathbb{Z})$ of index $q \geq 1$. Suppose that $\alpha \in \widetilde{L}$ is primitive in $L$.

(1) If $\beta \in L$ is a class such that $\Delta(\alpha, \beta)=1$, then $\widetilde{L}=\{j \alpha+$ $k q \beta \mid j, k \in \mathbb{Z}\}$.

(2) $\gamma \in \widetilde{L}$ if and only if $\Delta(\alpha, \gamma) \equiv 0(\bmod q)$.

Proof. Since $\alpha$ and $q \beta$ are both in $\widetilde{L}$ and span a sublattice of $L$ of index $q$, part (1) holds. To prove part (2), let $\gamma=j \alpha+m \beta \in L$. Then $\Delta(\alpha, \gamma)=|m|$. In particular, $\Delta(\alpha, \gamma) \equiv 0(\bmod q)$ if and only if $|m| \equiv 0(\bmod q)$. By part $(1)$ the latter holds if and only if $\gamma \in \widetilde{L}$.

q.e.d.

For an irreducible complex affine curve $C$, we denote its smooth projective model by $\widetilde{C}$. Note that $\widetilde{C}$ is birationally equivalent to $C$ and that any birational equivalence between them induces an isomorphism between the function fields $\mathbb{C}(C)$ and $\mathbb{C}(\widetilde{C})$. Thus any rational function 
$f$ on $C$ corresponds to a rational function $\widetilde{f}$ on $\widetilde{C}$. For $\widetilde{f} \in \mathbb{C}(\widetilde{C})$ and point $x \in \widetilde{C}$, we use $Z_{x}(\widetilde{f})$ to denote the multiplicity of $x$ as a zero of $\widetilde{f}$. The multiplicity of $x$ as a pole of $\widetilde{f}$ will be denoted by $\Pi_{x}(\widetilde{f})$.

A birational equivalence from $\widetilde{C}$ to $C$ is regular at all but a finite number of points of $\widetilde{C}$, called ideal points of $\widetilde{C}$. Normalization [30, Chapter II, §5] determines a surjective regular map $\nu: C^{\nu} \rightarrow C$ of $C$ where $C^{\nu}$ is a nonsingular affine set which can be identified with the subset of $\widetilde{C}$ whose complement $\widetilde{C} \backslash C^{\nu}$ is the set of ideal points of $\widetilde{C}$.

Let $C$ be an affine curve having $n$ irreducible components $C_{1}, \ldots, C_{n}$ and set $\widetilde{C}=\widetilde{C}_{1} \sqcup \cdots \sqcup \widetilde{C}_{n}, C^{\nu}=C_{1}^{\nu} \sqcup \cdots \sqcup C_{n}^{\nu}$ (here "ப" denotes disjoint union). An ideal point of $\widetilde{C}$ is an ideal point of $\widetilde{C}_{i}$ for some $i$, i.e., a point of $\widetilde{C} \backslash C^{\nu}$.

A point $x$ on a complex, affine, algebraic set $X$ is called a simple point if it is contained in a unique algebraic component $X_{0}$ of $X$ and is a smooth point of $X_{0}$ [30]. The point $x$ is simple on $X$ if and only if the dimension of the Zariski tangent space of $X$ at $x$ is equal to the dimension of some irreducible component of $X$ which contains $x$.

\section{The type of a finite filling slope}

In order to develop the theory of finite filling classes in the most useful way, we need to refine our notion of the type of a finite filling slope.

Recall that a representation $\rho \in R(G)$ is reducible if $\rho(G)$ can be conjugated into the set of upper triangular matrices. We call a representation $\rho \in R(G)$ virtually reducible if there is a finite index subgroup $\widetilde{G}$ of $G$ such that the restriction of $\rho$ to $\widetilde{G}$ is reducible. The reducibility of a representation in $R(G)$ is determined by its character [12, Corollary 1.2.2] and so we call $\chi \in X(G)$ reducible, or virtually reducible, if it is the character of a representation having that property. A representation or character which is not reducible is called irreducible.

Suppose that $\alpha$ is a finite filling class and that $\bar{\rho}: \pi_{1}(M(\alpha)) \rightarrow$ $P S L(2, \mathbb{C})$ is an irreducible representation. Let $T_{12}, I_{60}, O_{24}$ and $D_{n}$ denote, respectively, the tetrahedral group, the icosahedral group, the octahedral group, and the dihedral group of order $2 n$. It follows from 
$[2, \S 5]$ that

$$
\operatorname{image}(\bar{\rho}) \cong \begin{cases}D_{n}, \text { for some } n \geq 2 & \text { if } \alpha \text { is } D \text { or } Q \text {-type } \\ T_{12} & \text { if } \alpha \text { is } T \text {-type } \\ I_{60} & \text { if } \alpha \text { is } I \text {-type } \\ O_{24} \text { or } D_{3} & \text { if } \alpha \text { is } O \text {-type. }\end{cases}
$$

Let $\bar{\psi}$ be the composition $\pi_{1}(M) \rightarrow \pi_{1}(M(\alpha)) \stackrel{\bar{\rho}}{\rightarrow} P S L(2, \mathbb{C})$ and define $\bar{\phi}=\bar{\psi} \mid \pi_{1}(\partial M)$.

Lemma 3.1. Let $\bar{\rho}, \bar{\psi}$, and $\bar{\phi}$ be as above and set $q=\left|\bar{\phi}\left(\pi_{1}(\partial M)\right)\right|$. If $\alpha$ has $O$-type assume that image $(\bar{\rho}) \cong O_{24}$. Then:

(1) $q \in\{1,2\}$ if $\alpha$ is $D$ or $Q$-type and $\bar{\psi}$ can be arbitrarily closely approximated on $\bar{R}(M)$ by non-virtually reducible representations.

(2) (a) $q=3$ if $\alpha$ is T-type and $H_{1}(M ; \mathbb{Z})$ has no 3-torsion.

(b) $q \in\{1,2\}$ if $\alpha$ is $T$-type and $H_{1}(M ; \mathbb{Z})$ has nontrivial 3torsion.

(3) $q \in\{1,2,3,5\}$ if $\alpha$ is I-type.

(4) (a) $q \in\{2,4\}$ if $\alpha$ is $O$-type and $H_{1}(M ; \mathbb{Z})$ has no 2-torsion.

(b) $q \in\{1,2,3\}$ if $\alpha$ is $O$-type and $H_{1}(M ; \mathbb{Z})$ has nontrivial 2torsion.

Proof. Suppose first of all that $\alpha$ is of type $T$. Since $\phi\left(\pi_{1}(\partial M)\right)$ is cyclic and is a subgroup of $T_{12}$, it has order $q \in\{1,2,3\}$. If $H_{1}(M ; \mathbb{Z})$ has no 3 -torsion then $H_{1}\left(\partial M ; \mathbb{Z}_{3}\right) \rightarrow H_{1}\left(M ; \mathbb{Z}_{3}\right) \cong \mathbb{Z}_{3}$ is surjective, and hence the composition $H_{1}\left(\partial M ; \mathbb{Z}_{3}\right) \rightarrow H_{1}\left(M ; \mathbb{Z}_{3}\right) \rightarrow H_{1}\left(M(\alpha) ; \mathbb{Z}_{3}\right) \stackrel{\sigma}{\rightarrow}$ $H_{1}\left(T_{12} ; \mathbb{Z}_{3}\right)$ is as well, where $\sigma$ is the homomorphism induced by $\bar{\rho}$. It follows that the cyclic group $\phi\left(\pi_{1}(\partial M)\right)$ does not lie in $\left[T_{12}, T_{12}\right] \cong$ $\mathbb{Z}_{2} \oplus \mathbb{Z}_{2}$, and therefore $\phi\left(\pi_{1}(\partial M)\right) \cong \mathbb{Z}_{3}$, i.e., $q=3$.

Suppose next that $\alpha$ is of type $T$ and $H_{1}(M ; \mathbb{Z})$ has nontrivial 3 torsion. Then $H_{1}\left(M ; \mathbb{Z}_{3}\right) \cong A \oplus B$ where $A=\operatorname{image}\left(H_{1}\left(\partial M ; \mathbb{Z}_{3}\right) \rightarrow\right.$ $\left.H_{1}\left(M ; \mathbb{Z}_{3}\right)\right) \cong \mathbb{Z}_{3} \cong B$. Since $\mathbb{Z}_{3} \cong H_{1}\left(M(\alpha) ; \mathbb{Z}_{3}\right) \cong(A /\langle\operatorname{image}(\alpha)\rangle) \oplus$ $B$ we see that the composition $H_{1}\left(\partial M ; \mathbb{Z}_{3}\right) \rightarrow H_{1}\left(M(\alpha) ; \mathbb{Z}_{3}\right)$ is zero. This implies that $\bar{\phi}\left(\pi_{1}(\partial M)\right) \subset\left[T_{12}, T_{12}\right] \cong \mathbb{Z}_{2} \oplus \mathbb{Z}_{2}$, and therefore $q \in\{1,2\}$.

The cases where $\alpha$ is of type $I$ or $O$ are handled in a similar fashion.

Next assume that $\alpha$ is $D$ or $Q$-type. The argument here is necessarily more involved as the torsion in the image of $\bar{\rho}$ has arbitrarily high order. 
Without loss of generality we may suppose that $q>1$. Now $\bar{\psi}$ is a point on $\bar{R}(M)$, the $P S L(2, \mathbb{C})$-representation variety of $\pi_{1}(M)$ ([3]). Arguing as in Lemmas 4.3 and 4.4 of [2], it can be shown that the dimension of the Zariski tangent space of $\bar{R}(M)$ at $\bar{\psi}$ is 4 . It follows that $\bar{\psi}$ is a simple point of $\bar{R}(M)$ (cf. $\S 2)$ and hence $X_{0}$, the component of the $P S L(2, \mathbb{C})$-character variety of $\pi_{1}(M)$ which contains the character of $\bar{\psi}$, is a curve. The hypotheses of part (1) of this lemma imply that $X_{0}$ contains non-virtually reducible characters. The argument in the proof of $[2$, Theorem 2.1] can then be adapted to a $P S L(2, \mathbb{C})$ setting to see that there is an index 2 sublattice $\widetilde{L}$ of $L$, which contains $\alpha$, on which $\bar{\phi}$ is trivial (compare [2, Lemma $6.1(3)]$ ). Thus $q=2$. $\quad$ q.e.d.

Definition 3.2. Suppose that $\alpha$ is a $T, I$ or $O$-type class and fix an irreducible representation $\bar{\rho}: \pi_{1}(M(\alpha)) \rightarrow P S L(2, \mathbb{C})$ whose image is $O_{24}$ if $\alpha$ has type $O$. According to [2, Lemma 5.3], $\bar{\rho}$ is well-defined up to conjugacy, and an outer automorphism of its image when $\alpha$ is of type $I$. If $\bar{\phi}$ denotes the composition $\pi_{1}(\partial M) \rightarrow \pi_{1}(M) \rightarrow \pi_{1}(M(\alpha)) \stackrel{\bar{\rho}}{\rightarrow}$ $P S L(2, \mathbb{C})$, we shall say that a finite filling class has type $T(q)$ if it is of type $T$ and $q=\left|\bar{\phi}\left(\pi_{1}(\partial M)\right)\right|$. Similarly we define $I(q)$-type and $O(q)$-type filling classes.

A simple consequence of these definitions is the following useful result.

Proposition 3.3. Suppose that $\alpha$ is a finite filling class of type $T(q), I(q)$ or $O(q)$. If $\beta \in L$ is another finite filling class such that $\Delta(\alpha, \beta) \equiv 0(\bmod q)$, then it also has, respectively, type $T(q), I(q)$ or $O(q)$.

Proof. Say $\alpha$ has type $T(q)$ and fix a representation $\bar{\rho}: \pi_{1}(M(\alpha)) \rightarrow$ $P S L(2, \mathbb{C})$ whose image is the tetrahedral group $T_{12}$. If we denote by $\phi$ the composition $\pi_{1}(\partial M) \rightarrow \pi_{1}(M) \rightarrow \pi_{1}(M(\alpha)) \stackrel{\bar{\rho}}{\rightarrow} T_{12}$, then from the definition of $q$ and the hypothesis that $\Delta(\alpha, \beta) \equiv 0(\bmod q)$ we deduce that $\phi(\beta)= \pm I$. Thus the composite $\pi_{1}(M) \rightarrow \pi_{1}(M(\alpha)) \stackrel{\bar{\phi}}{\rightarrow} T_{12}$ factors through $\pi_{1}(M(\beta))$. It is shown in [2, Lemma 5.3] that if the fundamental group of a 3-manifold is finite and admits a homomorphism onto the tetrahedral group, then it is $T$-type. From the definition of $q$ we now see that $\beta$ is actually of type $T(q)$.

q.e.d. 


\section{Norm curve components of $X(M)$}

In this section we collect some basic facts and properties of the norm curve components of the $S L(2, \mathbb{C})$-character variety of $M$, and of the Culler-Shalen norms which they determine on the 2-dimensional real vector space $H_{1}(\partial M ; \mathbb{R})$. These norms were originally defined in [11] and applied to the study of cyclic and finite fillings in [11] and [2]. See [3] for a discussion of Culler-Shalen norms in the setting of the $P S L(2, \mathbb{C})$-character variety of $M$.

For a finitely generated group $G$, we use $R(G)$ to denote the set of all representations of $G$ with values in $S L_{2}(\mathbb{C})$. It is well known that $R(G)$ has the structure of a complex affine algebraic set [12]. The character of an element $\rho \in R(G)$ is the function $\chi_{\rho}: G \rightarrow \mathbb{C}$ defined by $\chi_{\rho}(g)=\operatorname{trace}(\rho(g))$. The set of characters of the representations in $R(G)$, denoted by $X(G)$, is also a complex affine algebraic set [12] and is called the $S L(2, \mathbb{C})$-character variety of $G$. The surjective map $t: R(G) \rightarrow X(G)$ which sends a representation to its character is regular in the sense of algebraic geometry. For a compact manifold $W, R(W)$ and $X(W)$ will denote $R\left(\pi_{1}(W)\right)$ and $X\left(\pi_{1}(W)\right)$ respectively.

For each $g \in G$, the evaluation map $I_{g}: X(G) \rightarrow \mathbb{C}$ defined by $I_{g}\left(\chi_{\rho}\right)=\chi_{\rho}(g)$ is regular [12] and so $f_{g}=\left(I_{g}+2\right)\left(I_{g}-2\right)$ is as well. It is easy to verify that $I_{g}$, and hence $f_{g}$, is unchanged if we replace $g$ by its inverse or any of its conjugates in $G$.

Consider a hyperbolic knot exterior $M$. The Hurewicz homomorphism induces an isomorphism $H_{1}(\partial M ; \mathbb{Z}) \cong \pi_{1}(\partial M)$, and so we can identify $L=H_{1}(\partial M ; \mathbb{Z})$ with a subgroup of $\pi_{1}(M)$, well-defined up to conjugacy. Each $\delta \in L$ therefore determines a regular function $I_{\delta}: X(M) \rightarrow \mathbb{C}$. An irreducible 1-dimensional algebraic component $X_{1}$ of $X(M)$ is called a norm curve component (for reasons to be made clear below) if $I_{\delta}$ is nonconstant on $X_{1}$ for every $\delta \in L \backslash\{0\}$. Since $M$ is an orientable hyperbolic manifold, $X(M)$ contains the characters of discrete faithful representations [12, Proposition 3.1.1]. It is proven in [11, Proposition 1.1.1] that any irreducible component of $X(M)$ containing such a character is a norm curve component of $X(M)$.

Proposition 4.1. Let $X_{1}$ be a norm curve component of $X(M)$. Then all but finitely many characters in $X_{1}$ are irreducible.

Proof. It follows from Lefschetz duality that the rank of the natural homomorphism $H_{1}(\partial M ; \mathbb{Z}) \rightarrow H_{1}(M ; \mathbb{Z})$ is 1 , and so there is a nonzero class $\alpha \in H_{1}(\partial M ; \mathbb{Z})$ which is homologically trivial in $M$. Thus if $\chi$ is 
the character of an abelian representation, we have $\chi(\alpha)=2$.

Now every reducible character in $X(M)$ is easily seen to be the character of a diagonal, and therefore abelian, representation of $\pi_{1}(M)$. Hence it follows from the previous paragraph that if $X_{1}$ contains infinitely many reducible characters, then $I_{\alpha} \mid X_{1}$ is constantly equal to 2 , contradicting the assumption that $X_{1}$ is a norm curve. Thus we are done.

q.e.d.

The set of virtually reducible characters in a norm curve is also constrained. Our next result shows that if this set is infinite, then the norm curve consists of the characters of very special collection of representations.

Let $D$ denote the set of diagonal matrices in $S L(2, \mathbb{C})$ and consider the group

$$
N=\left\{\left(\begin{array}{cc}
z & 0 \\
0 & z^{-1}
\end{array}\right),\left(\begin{array}{cc}
0 & w \\
-w^{-1} & 0
\end{array}\right) \mid z, w \in \mathbb{C}^{*}\right\} .
$$

which contains $D$ as an index 2 subgroup.

Proposition 4.2. Let $X_{1}$ be a norm curve component of $X(M)$ which contains infinitely many virtually reducible characters. Then there is an index 2 subgroup $\widetilde{\pi} \subset \pi_{1}(M)$ such that $\chi \mid \widetilde{\pi}$ is reducible for each $\chi \in X_{1}$. Indeed each element of $X_{1}$ is the character of a representation $\rho: \pi_{1}(M) \rightarrow N$ and $\widetilde{\pi}=\rho^{-1}(D)$ for the generic $\chi_{\rho} \in X_{1}$.

Proof. Suppose that $\rho \in R(M)$ is irreducible and $\hat{\pi}$ is a finite index normal subgroup of $\pi_{1}(M)$ such that $\rho \mid \hat{\pi}$ is reducible, but not central. The non-centrality of $\rho \mid \hat{\pi}$ implies that there are at most two lines in $\mathbb{C}^{2}$ which are invariant under the $\hat{\pi}$-action determined by $\rho$. Since $\hat{\pi}$ is a normal subgroup of $\pi_{1}(M)$, these one or two lines are actually invariant under the action of $\pi_{1}(M)$ on $\mathbb{C}^{2}$ determined by $\rho$. The irreducibility of $\rho$ implies that there are exactly two lines $L_{1}, L_{2} \subset \mathbb{C}^{2}$ invariant under this action. Fix any $A \in S L_{2}(\mathbb{C})$ which takes $L_{1} \cup L_{2}$ to the coordinate axes. Then the image of $\rho_{1}=A \rho A^{-1}$ lies in $N$. It follows that $\tilde{\pi}=\rho_{1}^{-1}(D)$ is an index 2 subgroup of $\pi_{1}(M)$ on which $\rho$ is diagonal.

Now suppose that $\left\{\chi_{1}, \chi_{2}, \chi_{3}, \ldots\right\} \subset X_{1}$ is an infinite set of virtually reducible characters. By Proposition 4.1 we may assume that each $\chi_{j}$ is the character of an irreducible representation $\rho_{j} \in R(M)$. We claim that we may assume that each $\rho_{j}$ conjugates into $N$. This follows from the preceding paragraph if we suppose that infinitely many of the $\rho_{j}$ have infinite image. Suppose then that each $\rho_{j}$ has finite image. From the 
classification of finite subgroups of $S L(2, \mathbb{C})$ (see e.g., [36, Lemma 2.6.5]) we see that $\rho_{j}\left(\pi_{1}(M)\right)$ is either the binary tetrahedral group, the binary octahedral group, the binary icosahedral group, or a binary dihedral group. There are only finitely many characters of representations in $R(M)$ of the first three types, so without loss of generality, the image of each $\rho_{j}$ is a binary dihedral group, and hence conjugate into $N$.

Assume then that each $\rho_{j}$ conjugates into $N$ and define $\tilde{\pi}_{j}=\rho_{j}^{-1}(D)$, an index 2 subgroup of $\pi_{1}(M)$. Since there are only finitely many such subgroups we may assume that $\widetilde{\pi}_{j}=\widetilde{\pi}_{k}$ for each $j, k \geq 1$. Then $\chi_{j} \mid \widetilde{\pi}_{1}$ is reducible for each $j$. But the set of characters in $X_{1}$ which are reducible when restricted to $\tilde{\pi}_{1}$ is an algebraic subset of $X_{1}$ (cf. [12, Corollary 1.2.2]) and therefore $\chi \mid \widetilde{\pi}_{1}$ is reducible for each $\chi \in X_{1}$. One may now argue, as in the first paragraph of the proof, that each irreducible character $\chi \in X_{1}$ is the character of a representation $\rho \in R(M)$ for which $\rho\left(\pi_{1}(M)\right) \subset N$ and $\rho^{-1}(D)=\widetilde{\pi}_{1}$. The density of such characters in $X_{1}$ implies that the proposition holds. $\quad$ q.e.d.

Our next two corollaries show that norm curve components of $X(M)$ contain only finitely many virtually reducible characters as long as $M$ is the exterior of a knot in the 3 -sphere, or a manifold which admits a finite filling which has neither a cyclic nor dihedral fundamental group.

Corollary 4.3. Suppose that $X_{1}$ is a norm curve component of $X(M)$. If $H_{1}\left(M ; \mathbb{Z}_{2}\right) \cong \mathbb{Z}_{2}$, then $X_{1}$ contains only finitely many virtually reducible characters.

Proof. Suppose that $X_{1}$ contains infinitely many virtually reducible characters. According to Proposition 4.2, there is an index 2 subgroup $\tilde{\pi}$ of $\pi$ for which $\chi_{\rho}$ is reducible for each $\chi_{\rho} \in X_{1}$. Let $p: \widetilde{M} \rightarrow M$ be the 2 -fold cover determined by $\tilde{\pi}$. Restriction determines a regular map $p^{*}: X_{1} \rightarrow X(\widetilde{M})$ whose image determines a curve $Y_{1}$ in $X(\widetilde{M})$.

Let $\widetilde{T}$ be a boundary component of $\widetilde{M}$ and consider $\widetilde{\alpha} \in H_{1}(\widetilde{T} ; \mathbb{Z}) \backslash$ $\{0\}$. Since $X_{1}$ is a norm curve, the identity $I_{p_{*}(\widetilde{\alpha})}\left|X_{1}=I_{\widetilde{\alpha}}\right| Y_{1} \circ p^{*} \mathrm{im}-$ plies that $I_{\widetilde{\alpha}} \mid Y_{1}$ is nonconstant. Then by Proposition 4.1, $\partial \widetilde{M}$ cannot be connected and so $\pi_{1}(\partial M) \subset \pi_{1}(\widetilde{M})$. But then there is a surjection $\pi \rightarrow \pi / \widetilde{\pi}=\mathbb{Z}_{2}$ which vanishes on $\pi_{1}(\partial M)$. This is impossible as the hypothesis that $H_{1}\left(M ; \mathbb{Z}_{2}\right) \cong \mathbb{Z}_{2}$ implies that $H_{1}\left(\partial M ; \mathbb{Z}_{2}\right) \rightarrow H_{1}\left(M ; \mathbb{Z}_{2}\right)$ is surjective. Thus there are only finitely many virtually reducible characters in $X_{1}$.

q.e.d. 
Corollary 4.4. Suppose that $X_{1}$ is a norm curve component of $X(M)$ and that it contains the character of a representation $\rho$ with finite image which is neither cyclic nor binary dihedral. Then $X_{1}$ contains only finitely many virtually reducible characters.

Proof. Our hypothesis on $\rho$ implies that it does not conjugate into $N$. Thus the corollary follows from Proposition 4.2.

q.e.d.

Now consider a norm curve component $X_{1}$ of $X(M)$. The method of $[11, \S 1.4]$ can be used to define a norm $\|\cdot\|_{1}$ on $H_{1}(\partial M ; \mathbb{R})$ which satisfies (and is determined by) the identity

$$
\|\delta\|_{1}=\operatorname{degree}\left(f_{\delta} \mid X_{1}\right)=2 \operatorname{degree}\left(I_{\delta} \mid X_{1}\right) \text { for each element } \delta \in L .
$$

Our next proposition lists some of the basic properties of this norm.

\section{Proposition 4.5.}

(1) Let

$$
\begin{aligned}
s_{1} & =\min \left\{\|\delta\|_{1} \mid \delta \in L \backslash\{0\}\right\} \\
B_{1} & =\left\{v \in V \mid\|v\|_{1} \leq s_{1}\right\} .
\end{aligned}
$$

Then $B_{1}$ is a compact, convex, finite-sided, balanced (i.e., $B_{1}=$ $\left.-B_{1}\right)$ polygon whose vertices are rational multiples of boundary classes in $L$. They are strict boundary classes if $X_{1}$ contains nonvirtually reducible characters.

(2) There are at most three (pairs of) classes of $L$ which lie on $\partial B_{1}$ but are not vertices. Their mutual distances are at most 1.

(3) Choose an ordered basis $\{\mu, \lambda\}$ for $L$ such that $\|\mu\|_{1}=s_{1}$ and identify $V$ with the $\mu \lambda$-plane (as discussed in $\S 2$ ). Then if $(a, b) \in$ $B_{1}$, we have $|b| \leq 2$. Moreover, if there is some $(a, b) \in B_{1}$ with $b=2$, then $(a, b) \in L$ and $B_{1}$ is a parallelogram with vertices $\pm(1,0)$ and $\pm(a, b)$.

Proof. Part (1) is proved as in [11, $§ 1.4]$, though see [11, Proposition 1.2.7] and [3, Proposition $5.2(5)]$ for the strictness of the boundary slopes associated to vertices of $B_{1}$ when $X_{1}$ contains the character of a non-virtually reducible representation. Part (2) can be found in [11, $\S 1.1]$, while part (3) is proved in [2, Lemma 6.4].

It was shown in [11] and [2] how to obtain restrictions on the norms of finite or cyclic filling classes, which are not strict boundary classes, 
from norm curves which contain the character of a discrete, faithful representation. These results can be extended to general norm curves.

Proposition 4.6. Suppose that $X_{1}$ is a norm curve component and that $\alpha=(m, n) \in L$ is a finite or cyclic filling class which is not a strict boundary class.

(1) If $\alpha \in L$ is a cyclic filling class, and $X_{1}$ contains a character which is not virtually reducible, then $\|\alpha\|_{1}=s_{1}$. Hence $\alpha \in \partial B_{1}$ but is not a vertex of $B_{1}$.

(2) If $\alpha$ is a D-type or a $Q$-type filling class and $X_{1}$ contains a character which is not virtually reducible, then

(i) $\|\alpha\|_{1} \leq 2 s_{1}$,

(ii) $\|\alpha\|_{1} \leq\|\beta\|_{1}$

for any nonzero element $\beta \in L$ such that $\Delta(\alpha, \beta) \equiv 0(\bmod 2)$.

(3) (a) If $\alpha$ is a $T(q)$-type filling class and $H_{1}(M ; \mathbb{Z})$ has no 3 torsion, then $q=3$ and

(i) $\|\alpha\|_{1} \leq s_{1}+4$,

(ii) $\|\alpha\|_{1} \leq\|\beta\|_{1}$

for any nonzero element $\beta \in L$ such that $\Delta(\alpha, \beta) \equiv$ $0(\bmod q)$.

(b) If $\alpha$ is a $T(q)$-type filling class and $H_{1}(M ; \mathbb{Z})$ has nontrivial 3-torsion, then $q \in\{1,2\}$ and

(i) $\|\alpha\|_{1} \leq s_{1}+4$,

(ii) $\|\alpha\|_{1} \leq\|\beta\|_{1}$

for any nonzero element $\beta \in L$ such that $\Delta(\alpha, \beta) \equiv$ $0(\bmod q)$. If $\|\alpha\|_{1}>s_{1}$, then $q=2$.

(4) If $\alpha$ is an I( $q)$-type filling class, then $q \in\{1,2,3,5\}$ and

(i) $\|\alpha\|_{1} \leq s_{1}+8$,

(ii) $\|\alpha\|_{1} \leq\|\beta\|_{1}$

for any nonzero element $\beta \in L$ such that $\Delta(\alpha, \beta) \equiv 0(\bmod q)$. If $\|\alpha\|_{1}>s_{1}$, then $q>1$.

(5) (a) If $\alpha$ is an $O(q)$-type filling class and $H_{1}(M ; \mathbb{Z})$ has no 2torsion, then $q \in\{2,4\}$, and 
(i) $\|\alpha\|_{1} \leq s_{1}+6$,

(ii) $\|\alpha\|_{1} \leq\|\beta\|_{1}$

for any nonzero element $\beta \in L$ such that $\Delta(\alpha, \beta) \equiv$ $0(\bmod q)$.

(b) If $\alpha$ is an $O(q)$-type filling class and $H_{1}(M ; \mathbb{Z})$ has nontrivial 2-torsion, then $q \in\{1,2,3\}$, and

(i) $\|\alpha\|_{1} \leq s_{1}+12$,

(ii) $\|\alpha\|_{1} \leq\|\beta\|_{1}$

for any nonzero element $\beta \in L$ such that $\Delta(\alpha, \beta) \equiv$ $0(\bmod q)$. If $\|\alpha\|_{1}>s_{1}$, then $q>1$.

Proof. Most of the details of the argument can be found in the proof of [2, Theorem 2.3], taking into account Lemma 2.3, Proposition 4.1, and Corollary 4.4 of this paper. The fact that $q>1$ when $\|\alpha\|_{1}>s_{1}$ follows from [2, Lemmas 4.1, 4.2, and 5.3].

q.e.d.

Corollary 4.7. Suppose that $\alpha$ and $\beta$ are finite filling classes, but not strict boundary classes. If $\alpha$ is of type $T(q), I(q)$ or $O(q)$ and $\Delta(\alpha, \beta) \equiv 0(\bmod q)$, then $\|\beta\|_{1}=\|\alpha\|_{1}$.

Proof. The result follows immediately from Propositions 3.3 and 4.6.

q.e.d.

The final result of this section will prove useful in the proof of the finite surgery conjecture.

Corollary 4.8. Suppose that $X_{1}$ is a norm curve component which contains a non-virtually reducible character. Choose a basis $\{\mu, \lambda\}$ for $L$ such that $\|\mu\|_{1}=s_{1}$ and identify $H_{1}(\partial M ; \mathbb{R})$ with the $\mu \lambda$-plane. If $\alpha=(j, 2)$ is a finite or cyclic filling class, but is not a strict boundary class, and $q \leq 2$, then $\alpha$ is neither a $C, D, Q, T(q), O(q)$ or $I(q)$-type filling class.

Proof. Suppose otherwise. Then there is an integer $m$ such that $\alpha=(2 m+1,2)$ is, say, a $D$-type filling class (the other classes can be treated similarly). Since $\Delta(\alpha, \mu)=2$, Lemma 2.1 implies that both $\alpha$ and $\mu$ are contained in the index two sublattice $\widetilde{L}$ of $L$ described in Proposition 4.6 (2). Thus $\|\alpha\|_{1} \leq\|\mu\|_{1}=s_{1}$, i.e., $\alpha=(2 m+1,2) \in \partial B_{1}$. Hence Proposition $4.5(3)$ implies that $(2 m+1,2)$ is a vertex of $B_{1}$, and thus is a strict boundary class by Proposition 4.5 (2). But this is contrary to our assumption.

q.e.d. 


\section{The $\operatorname{Aut}(\mathbb{C}) \times H^{1}\left(M ; \mathbb{Z}_{2}\right)$-action}

Let $\operatorname{Aut}(\mathbb{C})$ denote the group of field automorphisms of the complex numbers. As we shall see below, the group $\operatorname{Aut}(\mathbb{C}) \times H^{1}\left(M ; \mathbb{Z}_{2}\right)$ acts in a natural fashion on the set of norm curve components of $X(M)$. A key fact for us is that the orbits of this action are defined over the rationals.

The group $\operatorname{Aut}(\mathbb{C})$ acts on both $\mathbb{C}^{n}$ and the ring $\mathbb{C}\left[z_{1}, z_{2}, \ldots, z_{n}\right]$ : for $\phi \in \operatorname{Aut}(\mathbb{C}),\left(c_{1}, c_{2}, \ldots, c_{n}\right) \in \mathbb{C}^{n}$, and polynomial

$$
\sum_{m_{*}} a_{m_{*}} z_{1}^{m_{1}} z_{2}^{m_{2}} \ldots z_{n}^{m_{n}} \in \mathbb{C}\left[z_{1}, z_{2}, \ldots, z_{n}\right]
$$

we have

$$
\begin{aligned}
\phi\left(c_{1}, c_{2}, \ldots, c_{n}\right) & =\left(\phi\left(c_{1}\right), \phi\left(c_{2}\right), \ldots, \phi\left(c_{n}\right)\right) \\
\phi\left(\sum_{m_{*}} a_{m_{*}} z_{1}^{m_{1}} z_{2}^{m_{2}} \ldots z_{n}^{m_{n}}\right) & =\sum_{m_{*}} \phi\left(a_{m_{*}}\right) z_{1}^{m_{1}} z_{2}^{m_{2}} \ldots z_{n}^{m_{n}} .
\end{aligned}
$$

These actions are compatible in the sense that if $V(J)$ denotes the algebraic set associated to an ideal $J$ in $\mathbb{C}\left[z_{1}, z_{2}, \ldots, z_{n}\right]$, then

$$
\phi(V(J))=V(\phi(J))
$$

for each $\phi \in \operatorname{Aut}(\mathbb{C})$. Similarly if $V \subset \mathbb{C}^{n}$ is an algebraic set and $J(V) \subset \mathbb{C}\left[z_{1}, z_{2}, \ldots, z_{n}\right]$ is its ideal, then

$$
\phi(J(V))=J(\phi(V)) .
$$

Algebraically definable notions such as "dimension", "irreducible", "component", "simple point", etc. are preserved by the actions.

We say that an ideal $J \subset \mathbb{C}\left[z_{1}, z_{2}, \ldots, z_{n}\right]$ is defined over a subfield $\mathbb{K}$ of $\mathbb{C}$ if it is generated as a $\mathbb{C}\left[z_{1}, z_{2}, \ldots, z_{n}\right]$-module by polynomials $f_{1}, \ldots, f_{m} \in \mathbb{K}\left[z_{1}, z_{2}, \ldots, z_{n}\right]$. If $J$ is defined over $\mathbb{K}$ and each field over which $J$ is defined contains $\mathbb{K}$, we say that $\mathbb{K}$ is the minimal field of definition for $J$. The following theorem is due to André Weil (see e.g., [22, §III, Theorem 7 and $\S I I I .5])$.

Theorem 5.1 (Weil). Each ideal $J \subset \mathbb{C}\left[z_{1}, z_{2}, \ldots, z_{n}\right]$ has a minimal field of definition. Further, the algebraic set $V(J) \subset \mathbb{C}^{n}$ is invariant under $\phi \in \operatorname{Aut}(\mathbb{C})$ if and only if $\phi$ restricts to the identity on the minimal field of definition of $J$.

Corollary 5.2. An ideal $J \subset \mathbb{C}\left[z_{1}, z_{2}, \ldots, z_{n}\right]$ is defined over $\mathbb{Q}$ if and only if $V(J)$ is invariant under each automorphism of $\mathbb{C}$. 
Next consider a finitely generated group $G$. The action of Aut $(\mathbb{C})$ on $S L_{2}(\mathbb{C})$ given by

$$
\phi\left(\left(\begin{array}{ll}
a & b \\
c & d
\end{array}\right)\right)=\left(\begin{array}{ll}
\phi(a) & \phi(b) \\
\phi(c) & \phi(d)
\end{array}\right)
$$

determines actions of $\operatorname{Aut}(\mathbb{C})$ on $R(G)$ and $X(G)$ where for $\rho \in R(G)$ we have

$$
\phi(\rho)(g)=\phi(\rho(g))
$$

and for $\chi_{\rho} \in X(G)$,

$$
\phi\left(\chi_{\rho}\right)(g)=\chi_{\phi(\rho)}(g)=\phi\left(\chi_{\rho}(g)\right) .
$$

These actions are compatible with those described above under the algebraic embeddings of $R(G)$ and $X(G)$ into affine space discussed in [12]. Hence by Corollary 5.2, both $R(G)$ and $X(G)$ are defined over $\mathbb{Q}$. Indeed the same holds for the union of the algebraic sets in any Aut $(\mathbb{C})$-orbit of components of either $R(G)$ or $X(G)$.

Lemma 5.3. Let $X_{1} \in X(M)$ be a norm curve component of $X(M)$ and $\phi \in \operatorname{Aut}(\mathbb{C})$.

(1) Then $X_{2}=\phi\left(X_{1}\right)$ is also a norm curve component of $X(M)$. Indeed $\|\cdot\|_{2}=\|\cdot\|_{1}$. Thus the norm polygon $B_{1}$ defined by $X_{1}$ and the norm polygon $B_{2}$ defined by $X_{2}$ coincide.

(2) If $X_{1}$ contains a character which is not virtually reducible, then so does $X_{2}=\phi\left(X_{1}\right)$.

Proof. For each $\delta \in L$ there is a commutative diagram

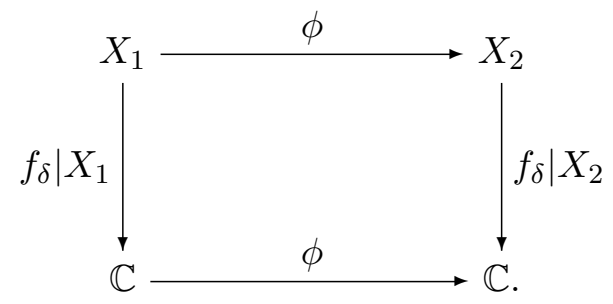

Since the degree of a map between curves is the cardinality of the inverse image of a generic point in its range, and since $\phi$ is a bijection (noncontinuous in general), we see that the degree of $f_{\delta} \mid X_{1}$ and that of $f_{\delta} \mid X_{2}$ coincide. Thus $\|\cdot\|_{1}=\|\cdot\|_{2}$. 
Part (2) of the lemma is easy to deduce, for if $\tilde{\pi}$ is a subgroup of $\pi_{1}(M)$ and $\rho \in R(M)$ restricts to a reducible representation on $\tilde{\pi}$, there is a line $L_{1}$ in $\mathbb{C}^{2}$ invariant under the $\widetilde{\pi}$-action determined by $\rho$. Then $\phi\left(L_{1}\right)$ is invariant under the $\widetilde{\pi}$-action determined by $\phi(\rho)$. Thus $\rho$ virtually reducible if and only if $\phi(\rho)=\phi \circ \rho$ is. $\quad$ q.e.d.

Two other actions of interest to us are those determined by $H^{1}\left(G ; \mathbb{Z}_{2}\right)$ $=\operatorname{Hom}(G,\{ \pm 1\})$ on $R(G)$ and $X(G)$. For $\epsilon \in H^{1}\left(G ; \mathbb{Z}_{2}\right), \rho \in R(G)$ and $\chi_{\rho} \in X(G)$ we define

$$
\epsilon(\rho)(g)=\epsilon(g) \rho(g)
$$

and

$$
\epsilon\left(\chi_{\rho}\right)(g)=\chi_{\epsilon(\rho)}(g)=\epsilon(g) \chi_{\rho}(g) .
$$

These actions are by algebraic isomorphisms and thus permute the algebraic components of $X(G)$, conserving dimension.

When $G=\pi_{1}(M)$, we shall identify $H^{1}\left(G ; \mathbb{Z}_{2}\right)$ with $H^{1}\left(M ; \mathbb{Z}_{2}\right)$.

Lemma 5.4. Let $X_{1} \in X(M)$ be a norm curve component and $\epsilon \in H^{1}\left(M ; \mathbb{Z}_{2}\right)$.

(1) Then $X_{2}=\epsilon\left(X_{1}\right)$ is also a norm curve component of $X(M)$. Indeed $\|\cdot\|_{2}=\|\cdot\|_{1}$. Thus the norm polygon $B_{1}$ defined by $X_{1}$ and the norm polygon $B_{2}$ defined by $X_{2}$ coincide.

(2) If $X_{1}$ contains a character which is not virtually reducible, then so does $X_{2}=\epsilon\left(X_{1}\right)$.

Proof. For each $\delta \in L$ there is a commutative diagram

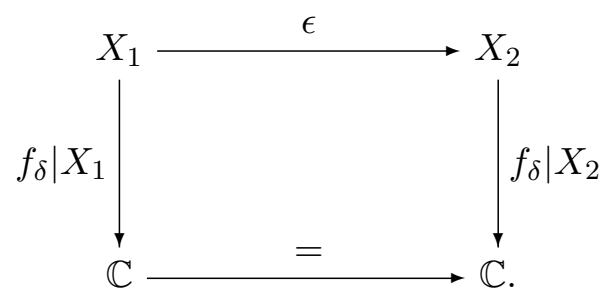

Since $\epsilon \mid X_{1}$ is an isomorphism, we see that $\|\delta\|_{1}=\|\delta\|_{2}$. Thus $\|\cdot\|_{1}=$ $\|\cdot\|_{2}$.

The conclusion of part (2) is obvious.

q.e.d.

The actions of Aut $(\mathbb{C})$ and of $H^{1}\left(M ; \mathbb{Z}_{2}\right)$ commute, and so there are combined actions of the direct product $\operatorname{Aut}(\mathbb{C}) \times H^{1}\left(G ; \mathbb{Z}_{2}\right)$ on $R(G)$ and $X(G)$. From Corollary 5.2 we derive the following proposition. 
Proposition 5.5. Let $Y$ be the union of the algebraic sets in an $\operatorname{Aut}(\mathbb{C}) \times H^{1}\left(G ; \mathbb{Z}_{2}\right)$-orbit of components of either $R(G)$ or $X(G)$. Then $Y$ is defined over $\mathbb{Q}$.

\section{A-polynomials}

In this section, we discuss the $A$-polynomial of $M$ associated to a norm curve component $X_{1}$ and defined with respect to a fixed ordered basis $\mathcal{B}=\{\mu, \lambda\}$ of $\pi_{1}(\partial M)$. The basic reference is [8]. Bearing in mind its use in the proof of the finite filling conjecture, it is most natural for us to choose a different normalization for the $A$-polynomial from that found in [8] — our $A$-polynomial is a power of theirs.

Fix a basis $\mathcal{B}=\{\mu, \lambda\}$ of $\pi_{1}(\partial M)$. Given a norm curve component $X_{1}$ of $X(M)$, one can construct an algebraic plane curve $D_{1}$ as follows. Let $i^{*}: X(M) \rightarrow X(\partial M)$ be the regular map induced by the inclusion induced homomorphism $i_{\#}: \pi_{1}(\partial M) \rightarrow \pi_{1}(M)$. Let $\Lambda$ be the set of diagonal representations of $\pi_{1}(\partial M)$, i.e.,

$$
\Lambda=\{\rho \in R(\partial M) \mid \rho(\mu), \rho(\lambda) \text { are diagonal matrices }\} .
$$

Then $\Lambda$ is a subvariety of $R(\partial M)$ and it is readily seen that $t \mid \Lambda: \Lambda \rightarrow$ $X(\partial M)$ is a degree 2 surjective map. We may identify $\Lambda$ with $\mathbb{C}^{*} \times$ $\mathbb{C}^{*}$ through use of the eigenvalue map $P_{\mathcal{B}}: \Lambda \rightarrow \mathbb{C}^{*} \times \mathbb{C}^{*}$. It sends a representation $\rho \in \Lambda$ to $(u, v) \in \mathbb{C}^{*} \times \mathbb{C}^{*}$ if $\rho(\mu)=\left(\begin{array}{cc}u & 0 \\ 0 & u^{-1}\end{array}\right)$ and $\rho(\lambda)=\left(\begin{array}{cc}v & 0 \\ 0 & v^{-1}\end{array}\right)$.

By hypothesis the function $I_{\mu}$ is nonconstant on $X_{1}$ and it clearly factors through $X(\partial M)$. Thus if $Y_{1}=\overline{i^{*}\left(X_{1}\right)}$ is the algebraic closure of $i^{*}\left(X_{1}\right)$ in $X(\partial M)$, then $Y_{1}$ is an irreducible curve in $X(\partial M)$. Next let $W_{1}$ denote the curve $\left.t\right|_{\Lambda} ^{-1}\left(Y_{1}\right)$ in $\Lambda$, and finally define $D_{1}$ to be the algebraic closure of $P_{\mathcal{B}}\left(W_{1}\right)$ in $\mathbb{C} \times \mathbb{C}$. The following diagram summarizes the construction.

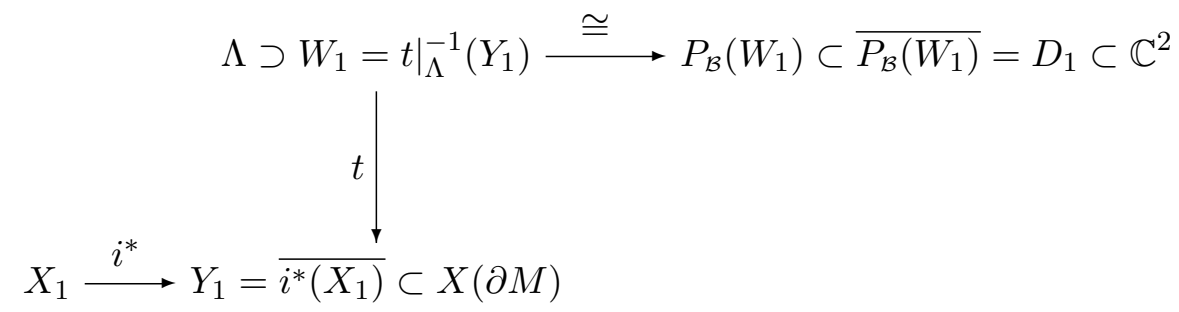


The curve $D_{1}$ is characterized by the conditon that generically speaking, a point $(u, v)$ lies on $D_{1}$ if and only if there is a representation $\rho \in R(M)$ with $\chi_{\rho} \in X_{1}$ such that $\rho \mid \pi_{1}(\partial M) \in \Lambda$ and the upper left hand entries of $\rho(\mu)$ and $\rho(\lambda)$ are $u$ and $v$ respectively.

Let $p_{1}(u, v)$ be a defining polynomial of $D_{1}$ with no repeated factors. Note that by construction, $D_{1}$ contains neither of the coordinate axes so that $p_{1}$ is not divisible by either $u$ or $v$. Set

$$
A_{1}(u, v)=p_{1}(u, v)^{d_{1}}
$$

where $d_{1}$ is the degree of the map $i^{*}: X_{1} \rightarrow Y_{1}$. We call $A_{1}(u, v)$ the $A$-polynomial of $X_{1}$ with respect to the basis $\mathcal{B}=\{\mu, \lambda\}$. Note that $A_{1}(u, v)$ is uniquely determined up to multiplication by a nonzero complex constant.

Remark 6.1. (1) In [8] the authors considered the $A$-polynomial of the whole character variety of $M$. Specifically, they proved that each algebraic component of $X(M)$ gives rise, by the process described above, to a plane algebraic set of dimension less than or equal to 1 . Let $D$ be the the union of the curves which arise from this process. The $A$-polynomial of [8] is a polynomial with no repeated factors which defines $D$. In particular they do not take into account the degree $d_{1}$ of $i^{*}: X_{1} \rightarrow X(\partial M)$. Thus in the notation above, our $p_{1}(u, v)$ is always a factor of their $A$-polynomial, but if $d_{1}>1$, our $A$-polynomial is not. Our choice of normalization is, of course, a matter of convenience, but it is also quite natural. This will become evident in $\S 8$.

(2) Very little seems to be known about the degree $d_{1}$ of $i^{*}: X_{1} \rightarrow$ $X(\partial M)$, though Nathan Dunfield has shown that if $X_{1}$ contains the character of a discrete, faithful representation, then $d_{1} \leq\left|H^{1}\left(M ; \mathbb{Z}_{2}\right)\right| / 2$ [15, Corollary 3.2].

Proposition $6.2([8])$. There are integers $r, s \geq 0$ such that

$$
A_{1}(u, v)=\epsilon u^{r} v^{s} A_{1}\left(u^{-1}, v^{-1}\right)
$$

where $\epsilon \in\{ \pm 1\}$.

Proof. This follows directly from the construction of $A_{1}(u, v)$. One verifies that two elements $\rho, \rho^{\prime} \in \Lambda$ have the same image under the map $\left.t\right|_{\Lambda}$ if and only if when $\rho(\mu)=\left(\begin{array}{cc}u & 0 \\ 0 & u^{-1}\end{array}\right)$ and $\rho(\lambda)=\left(\begin{array}{cc}v & 0 \\ 0 & v^{-1}\end{array}\right)$, then $\rho^{\prime}(\mu)=\left(\begin{array}{cc}u^{-1} & 0 \\ 0 & u\end{array}\right)$ and $\rho^{\prime}(\lambda)=\left(\begin{array}{cc}v^{-1} & 0 \\ 0 & v\end{array}\right)$. It follows that $D_{1}$ 
is invariant under the involution $(u, v) \mapsto\left(u^{-1}, v^{-1}\right)$. Hence there are integers $r, s \geq 0$ and a constant $c \in \mathbb{C}^{*}$ for which $u^{r} v^{s} A_{1}\left(u^{-1}, v^{-1}\right)=$ $c A_{1}(u, v)$. It is simple to deduce that $c \in\{ \pm 1\}$.

q.e.d.

Corollary 6.3. Suppose that $A_{1}(u, v)=\sum a_{m, n} u^{m} v^{n}$ is the $A$ polynomial of a norm curve component $X_{1}$ of $X(M)$. Let $m_{0}$ be the maximal exponent of $A_{1}(u, v)$ in $u$ and $n_{0}$ the maximal exponent in $v$. Then $a_{m, n}=\epsilon a_{m_{0}-m, n_{0}-n}$ for all $m$ and $n$ and some fixed constant $\epsilon \in\{ \pm 1\}$.

The Newton polygon of a two variable polynomial $p(u, v)=$ $\sum a_{m, n} u^{m} v^{n}$ is the convex hull in $\mathbb{R}^{2}$ of the set $\left\{(m, n) \mid a_{m, n} \neq 0\right\}$. A polygon in $\mathbb{R}^{2}$ is called balanced if it is invariant under reflection in some point of $\mathbb{R}^{2}$.

Proposition 6.4 ([8]). The Newton polygon $N_{1}$ of $A_{1}(u, v)$ is finite sided, convex and balanced. If $N_{1}$ has an edge of slope $q / p$, then $p \mu+q \lambda$ is a boundary class of the manifold $M$.

Proof. (Sketch). The first statement follows from the definition of the polygon and Corollary 6.3. The second is proved exactly as in [8]. The idea is that an appropriate Puiseaux parameterization of $D_{1}$ associated to the given edge determines the asymptotic behaviour of both $I_{\mu}$ and $I_{\lambda}$ at some ideal point of $X_{1}$. This behaviour was shown in [12] to determine a boundary class of $M$, which in this instance can be shown to be $p \mu+q \lambda$. (In $\S 6$, we will give another proof of this result which does not appeal to Puiseaux expansions.) q.e.d.

The $A$-polynomial has many interesting properties, one of the most remarkable we describe next. Let $N_{1}$ be the Newton polygon of the $A$-polynomial $A_{1}(u, v)=\sum a_{m, n} u^{m} v^{n}$ of a norm curve $X_{1}$. Fix an edge $E$ of $N_{1}$ having slope $q / p$, say, so that there is an integer $k$ for which $q m-p n=k$ for each $(m, n) \in E$. Then after taking appropriate roots we have

$$
\sum_{(m, n) \in E} a_{m, n} u^{m} v^{n}= \begin{cases}v^{\frac{-k}{p}} \sum_{(m, n) \in E} a_{m, n}\left(u v^{\frac{q}{p}}\right)^{m} & \text { if } p \neq 0 \\ u^{\frac{k}{q}} \sum_{(m, n) \in E} a_{m, n}\left(u^{\frac{p}{q}} v\right)^{n} & \text { if } q \neq 0 .\end{cases}
$$

The edge polynomials associated to $E$ are

$$
f_{E}(z)=\sum_{(m, n) \in E} a_{m, n} z^{m} \text { if } p \neq 0
$$


and

$$
g_{E}(z)=\sum_{(m, n) \in E} a_{m, n} z^{n} \quad \text { if } q \neq 0 .
$$

When both $p, q \neq 0$ it is easy to verify that $z_{0}$ is a nonzero root of $f_{E}$ if and only if there is a nonzero root $z_{1}$ of $g_{E}$ such that $z_{0}^{p}=z_{1}^{q}$. The significance of these roots is explained in the following proposition.

Proposition 6.5 ([8]). Let $E$ be an edge polynomial of the Newton polygon $N_{1}$. Then every root of an edge polynomial associated to $E$ is a root of the unity. Further if $\alpha=p \mu+q \lambda$ is the boundary class associated to the edge $E$ and $z_{0}$, respectively $z_{1}$, is a nonzero root of $f_{E}$, respectively $g_{E}$, then $f_{\alpha}$ takes on the value $\left(z_{0}^{p}-z_{0}^{-p}\right)^{2}$, respectively $\left(z_{1}^{q}-z_{1}^{-q}\right)^{2}$, at some ideal point of $\widetilde{X}_{1}$. In particular if one of \pm 1 is a root of either $f_{E}$ or $g_{E}$, then $f_{\alpha}$ takes on the value zero at some ideal point of $\widetilde{X}_{1}$.

We shall see in $\S 8$ that the $A$-polynomial of a norm curve component of $X(M)$ determines the associated Culler-Shalen norm. The proof will be based on our next result.

Proposition 6.6. Let $X_{1}$ be a norm curve component of $X(M)$ and suppose that $\|\mu\|_{1}=2 n_{1}$ and $\|\lambda\|_{1}=2 m_{1}$. Then $m_{1}$ is the largest power of $u$ which occurs in $A_{1}(u, v)$ while $n_{1}$ is the largest power of $v$.

Proof. We show that $\|\mu\|_{1}=2 n_{1}$ where $n_{1}$ is the largest power of $v$ in $A(u, v)$. The other equality is derived similarly.

Recall the plane curve $D_{1}$ associated to $X_{1}$ and the polynomial $p_{1}(u, v) \in \mathbb{C}[u, v]$, without repeated factors, which defines it. Consider the commutative diagram

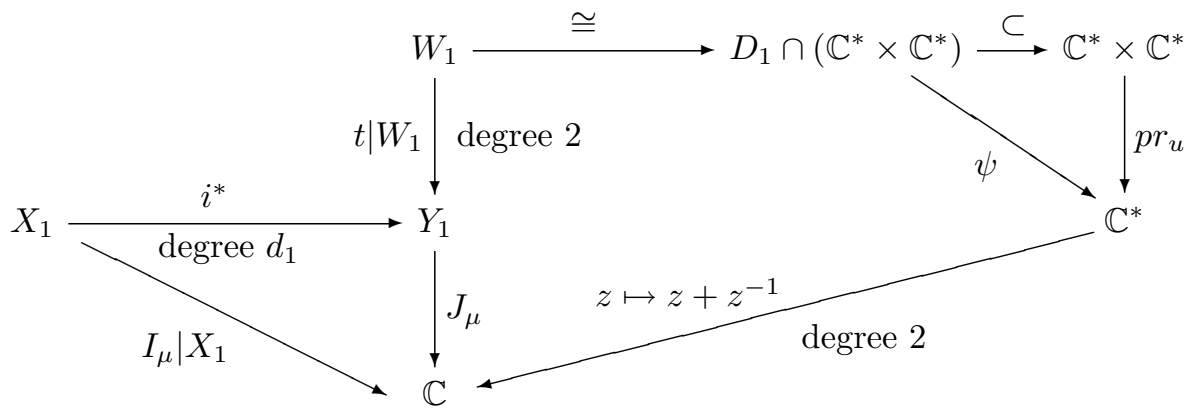

where $p r_{u}$ is projection on the first factor, $\psi$ is defined by the commutativity of the diagram, and $J_{\mu}: Y_{1} \rightarrow \mathbb{C}$ sends $\chi$ to $\chi(\mu)$. It follows that

$$
\|\mu\|_{1}=2 \operatorname{degree}\left(I_{\mu} \mid X_{1}\right)=2 d_{1} \operatorname{degree}(\psi) .
$$


Now degree $(\psi)$ is the cardinality of $\psi^{-1}\left(u_{0}\right)$ for a generic point $u_{0} \in \mathbb{C}^{*}$, and this, in turn, equals $\#\left\{v \in \mathbb{C}^{*} \mid p_{1}\left(u_{0}, v\right)=0\right\}$. To compute this quantity, think of $p_{1}$ as a polynomial in $v$, say

$$
p_{1}(u, v)=\sum_{s=0}^{k} h_{s}(u) v^{s} .
$$

Since $A_{1}=p_{1}^{d_{1}}$ we have $n_{1}=d_{1} k$. Expand $\frac{\partial p_{1}}{\partial v}$ in a similar fashion and consider the resultant $\operatorname{Res}_{v}\left(p_{1}, \frac{\partial p_{1}}{\partial v}\right) \in \mathbb{C}[u]$. Since $p_{1}$ has no repeated factors, this resultant is nonzero (see, for instance, [23, Corollary, $\S \mathrm{V} .10])$. From the properties of the resultant we see in particular that for the generic $u_{0} \in \mathbb{C}^{*}, p_{1}\left(u_{0}, v\right)$ is a polynomial of degree $k$ in $v$ with distinct roots. Hence degree $(\psi)=k$ and so from above

$$
\|\mu\|_{1}=2 \operatorname{degree}\left(I_{\mu} \mid X_{1}\right)=2 d_{1} \operatorname{degree}(\psi)=2 d_{1} k=2 n_{1} .
$$

q.e.d.

Corollary 6.7. If $\mu$ is not a boundary class parallel to an edge of the Newton polygon $N_{1}$. Then $A_{1}(1, v)$ is a polynomial of degree $\frac{\|\mu\|_{1}}{2}$ with nonzero constant term. Similarly $A_{1}(u, 1)$ is a polynomial of degree $\frac{\|\lambda\|_{1}}{2}$ with nonzero constant term.

Proof. We shall continue to use the notation in the proof of the previous proposition. Recall that $A_{1}(u, v)=p_{1}(u, v)^{d_{1}}=\left(\sum_{s=0}^{k} h_{s}(u) v^{s}\right)^{d_{1}}$ where $n_{1}=k d_{1}$. Thus

$$
A_{1}(u, v)=h_{0}(u)^{d_{1}}+\left(\text { terms involving } v, \ldots, v^{n_{1}-1}\right)+h_{k}(u)^{d_{1}} v^{n_{1}} .
$$

Since $\mu$ is not a boundary class, there is no edge of $N$ which is horizontal (Proposition 6.4). Hence $h_{0}$ and $h_{k}$ are nonzero constant polynomials. The desired result is then a consequence of this fact and Proposition 6.6.

q.e.d.

It is necessary to extend our notions to more general curves in $X(M)$. To that end let $C=X_{1} \cup \cdots \cup X_{k}$ where $X_{1}, X_{2}, \ldots, X_{k}$ are norm curve components of $X(M)$. Let $\|\cdot\|_{j}$ be the Culler-Shalen norm of $X_{j}$ and $A_{j}(u, v)$ its $A$-polynomial with respect to a basis $\{\mu, \lambda\}$ of $\pi_{1}(\partial M)$. Define the Culler-Shalen norm of $C$, denoted $\|\cdot\|_{\mathrm{C}}: H_{1}(\partial M ; \mathbb{R}) \rightarrow$ $[0, \infty)$, by

$$
\|\cdot\|_{\mathrm{C}}=\|\cdot\|_{1}+\|\cdot\|_{2}+\cdots+\|\cdot\|_{k}
$$


and the $A$-polynomial of $C$, with respect to $\{\mu, \lambda\}$, by

$$
A_{\mathrm{C}}(u, v)=A_{1}(u, v) A_{2}(u, v) \ldots A_{k}(u, v) .
$$

Note that $A_{\mathrm{C}}$ is well-defined up to multiplication by a nonzero complex constant. Of particular interest to us is the case where $C$ consists of the curves in an $\operatorname{Aut}(\mathbb{C}) \times H^{1}\left(M ; \mathbb{Z}_{2}\right)$ orbit.

Suppose that $X_{1}$ is a norm curve component of $X(M)$ and fix $(\phi, \epsilon) \in$ $\operatorname{Aut}(\mathbb{C}) \times H^{1}\left(M ; \mathbb{Z}_{2}\right)$. By Lemmas 5.3 and $5.4, X_{2}=(\phi, \epsilon)\left(X_{1}\right)$ is also a norm curve component of $X(M)$ and in fact $\|\cdot\|_{2}=\|\cdot\|_{1}$.

Next consider the $A$-polynomial of $X_{2}$ with respect to a fixed basis $\{\mu, \lambda\}$ of $\pi_{1}(\partial M)$. Let $D_{i}$ be the plane curve associated to $X_{i}$ and $p_{i}(u, v)$ a polynomial, without repeated factors, which defines it. From the construction of the plane curves $D_{i}$, it is not difficult to see that

$$
D_{2}=\left\{(\epsilon(\mu) \phi(u), \epsilon(\lambda) \phi(v)) \mid(u, v) \in D_{1}\right\},
$$

which we denote by $(\phi, \epsilon)\left(D_{1}\right)$. Hence we may take

$$
p_{2}(u, v)=\phi\left(p_{1}\right)(\epsilon(\mu) u, \epsilon(\lambda) v),
$$

which we denote by $(\phi, \epsilon)\left(p_{1}(u, v)\right)$. Let $d\left(X_{1}\right) \in \mathbb{Z}_{+}$denote the degree of the restriction map $X_{1} \rightarrow i^{*}\left(X_{1}\right) \subset X(\partial M)$ which is induced from the inclusion $i: \pi_{1}(\partial M) \rightarrow \pi_{1}(M)$.

Lemma 6.8. Suppose that $X_{1}$ is a norm curve component in $X(M)$. Then $d\left((\phi, \epsilon)\left(X_{1}\right)\right)=d\left(X_{1}\right)$.

Proof. Both $(\phi, \epsilon): X_{1} \rightarrow(\phi, \epsilon)\left(X_{1}\right)$ and $(\phi, \epsilon \circ i): i^{*}\left(X_{1}\right) \rightarrow$ $(\phi, \epsilon \circ i)\left(i^{*}\left(X_{1}\right)\right)$ are injective functions, so the conclusion is an immediate consequence of the commutativity of the following diagram.

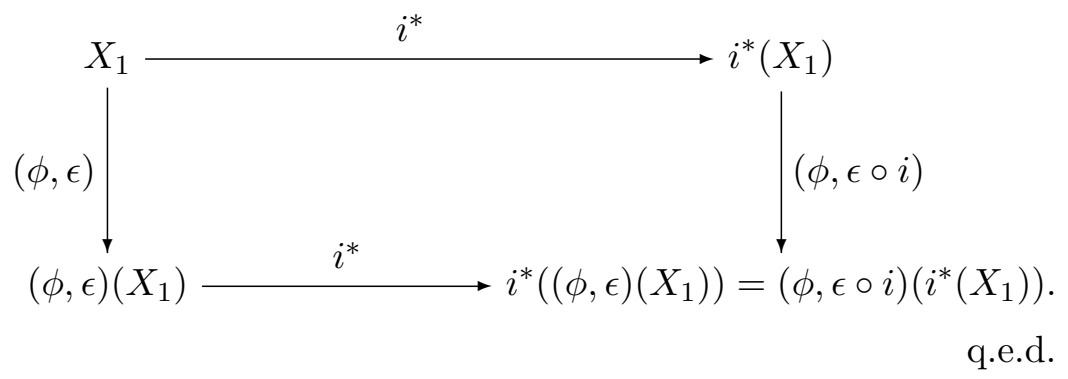

Corollary 6.9. Suppose that $X_{1}$ is a norm curve component of $X(M)$ and $(\phi, \epsilon) \in \operatorname{Aut}(\mathbb{C}) \times H^{1}\left(M ; \mathbb{Z}_{2}\right)$. If $X_{2}=(\phi, \epsilon)\left(X_{1}\right)$ then

$$
A_{2}(u, v)=\phi\left(A_{1}\right)(\epsilon(\mu) u, \epsilon(\lambda) v) \text {. }
$$


We finish this section with a proposition describing some of the main properties of the $A$-polynomial of an $\operatorname{Aut}(\mathbb{C}) \times H^{1}\left(M ; \mathbb{Z}_{2}\right)$-orbit of a norm curve component of $X(M)$.

Proposition 6.10. Suppose that $X_{1}$ is a norm curve component in $X(M)$. Let $X_{1}, X_{2}, \ldots, X_{k}$ be the distinct components in its $\operatorname{Aut}(\mathbb{C}) \times$ $H^{1}\left(M ; \mathbb{Z}_{2}\right)$-orbit. Set $C=X_{1} \cup X_{2} \cup \cdots \cup X_{k}$ and let $A_{C}(u, v)=$ $\sum a_{m, n} u^{m} v^{n}$ be the A-polynomial of $C$ with respect to a basis $\{\mu, \lambda\}$ of $\pi_{1}(\partial M)$. Let $N$ be the Newton polygon of $A_{C}$. Then:

(1) After multiplying by a nonzero complex constant, $A_{C}(u, v)$ may be taken to have integer coefficients whose greatest common denominator is 1 . Such a representative is well-defined up to sign.

(2) Let $m_{0}$ be the maximal exponent of $u$ occurring in $A_{C}(u, v)$ and $n_{0}$ that of $v$. Then for some $\epsilon \in\{ \pm 1\}, a_{m, n}=\epsilon a_{m_{0}-m, n_{0}-n}$ for all $m$ and $n$.

(3) The coefficients of $A_{C}(u, v)$ indexed by the corners of $N$ are equal to \pm 1 .

(4) The nonzero roots of any edge polynomial $f_{E}(z)$ or $g_{E}(z)$ determined by $A_{C}(u, v)$ are roots of the unity. In fact $f_{E}(z)$ and $g_{E}(z)$ are products of a power of $z$ with some cyclotomic polynomials. Further if $\alpha=(p, q)$ is the boundary class associated to the edge $E$ and if \pm 1 is a root of either $f_{E}(z)$ or $g_{E}(z)$, then $f_{\alpha}$ takes the value zero at some ideal point of $\widetilde{C}$.

(5) Let $i: \partial M \rightarrow M$ be the inclusion and $i_{*}: H_{1}\left(\partial M ; \mathbb{Z}_{2}\right) \rightarrow H_{1}\left(M ; \mathbb{Z}_{2}\right)$ the associated homomorphism.

(i) If $i_{*}(\mu) \neq 0$ and $i_{*}(\lambda)=0$, then $a_{m, n}=0$ when $m$ is odd.

(ii) If $i_{*}(\mu)=0$ and $i_{*}(\lambda) \neq 0$, then $a_{m, n}=0$ when $n$ is odd.

(iii) If $i_{*}(\mu) \neq 0$ and $i_{*}(\lambda) \neq 0$, then $a_{m, n}=0$ when $m+n$ is odd.

Proof. Let $D_{j}$ be the plane curve associated to $X_{j}$. Since $X_{1}, X_{2}$, $\ldots, X_{k}$ consists of several Aut($(\mathbb{C})$-orbits, the discussion prior to Lemma 6.8 implies that $D_{1}, D_{2}, \ldots, D_{k}$ consists of several Aut($(\mathbb{C})$-orbits as well, though note that there is a positive integer $r$, possibly larger than 1 , such that each orbit occurs $r$ times. It follows from Corollary 5.2 that $D_{1} \cup D_{2} \cup \cdots \cup D_{k}$ is defined over $\mathbb{Q}$, and so after multiplication by a suitable nonzero complex constant,

$$
p_{1}(u, v) p_{2}(u, v) \ldots p_{k}(u, v) \in \mathbb{Q}[u, v] .
$$


By Lemma 6.8,

$$
A_{\mathrm{C}}(u, v)=\Pi_{j=1}^{k} p_{j}(u, v)^{d\left(X_{j}\right)}=\left(\Pi_{j=1}^{k} p_{j}(u, v)\right)^{d_{1}} \in \mathbb{Q}[u, v] .
$$

After multiplying by a further nonzero rational constant we can assume that $A_{\mathrm{C}}(u, v) \in \mathbb{Z}[u, v]$ and that its coefficients have no common integer factors, other than \pm 1 . This determines $A_{\mathrm{C}}(u, v)$ up to sign. Part (1) of the lemma is therefore proved.

Next note that the conclusion of part (2) is a consequence of Proposition 6.2 and the identity $A_{\mathrm{C}}(u, v)=A_{1}(u, v) A_{2}(u, v) \ldots A_{k}(u, v)$.

For part (3), we observe that the proof of the main theorem in [10] applies verbatim to our situation, by the normalization we have chose in part (1) for $A_{\mathrm{C}}(u, v)$.

Part (4) follows from Proposition 6.5.

Finally consider part (5). From the definition of $C$ and Corollary 6.9 , it follows that $A(u, v)=A(\epsilon(\mu) u, \epsilon(\lambda) v)$ for any $\epsilon \in H^{1}\left(M ; \mathbb{Z}_{2}\right)$. If $\mu \notin \operatorname{ker}\left(H_{1}(\partial M ; \mathbb{Z}) \rightarrow H_{1}(M ; \mathbb{Z})\right)$ but $\lambda$ is, there is $\epsilon \in H^{1}\left(M ; \mathbb{Z}_{2}\right)$ such that $\epsilon(\mu)=-1$ and $\epsilon(\lambda)=1$. Hence $A(u, v)=A(-u, v)$ for each $(u, v) \in \mathbb{C}^{2}$. Therefore all odd exponents of $u$ in $A(u, v)$ must be zero. The other two cases are handled in a similar fashion.

q.e.d.

\section{Width}

In this section we define and study the width function of a polygon, originally introduced in [31]. It is the key ingredient to understanding the relationship between Culler-Shalen norms and the $A$-polynomial.

We consider $\mathbb{R}^{2}$ as a standard $u v$-plane. A polygon $N$ in $\mathbb{R}^{2}$ is called balanced if it is invariant under reflection in some point of $\mathbb{R}^{2}$. Two points on $N$ are called antipodes if they are related by such a reflection.

Let $N \subset \mathbb{R}^{2}$ be a convex, balanced polygon whose vertices lie in $\mathbb{Z}^{2}$. The width function $w=w_{N}: \mathbb{Q} \cup\{\infty\} \rightarrow \mathbb{Z}$ is defined by taking $w(q / p)=k$ if $k+1$ is the number of lines in the plane of slope $q / p$ which contain points of both $\mathbb{Z}^{2}$ and $N$.

The group $S L_{2}(\mathbb{Z})$ acts on both $\mathbb{R}$ and $\mathbb{R}^{2}$ in the following fashions. If $\Psi=\left(\begin{array}{ll}a & b \\ c & d\end{array}\right) \in S L_{2}(\mathbb{Z})$ then for $u \in \mathbb{R}$, we set $\Psi(u)=\frac{a u+b}{c u+d}$, and for $(u, v) \in \mathbb{R}^{2}$, we set $\Psi(u, v)=(a u+b v, c u+d v)$. Since $\Psi$ takes lines in $\mathbb{R}^{2}$ of slope $u \in \mathbb{R} \cup\{\infty\}$ to lines of slope $\left(\Psi\left(u^{-1}\right)\right)^{-1}$, we readily deduce the following lemma. 
Lemma 7.1. If $N$ is a convex, balanced polygon in $\mathbb{R}^{2}$ whose vertices lie in $\mathbb{Z}^{2}$ and $\Psi \in S L(2, \mathbb{Z})$, then $\Psi(N)$ is a convex, balanced polygon whose vertices lie in $\mathbb{Z}^{2}$ and

$$
w_{\Psi(N)}\left((\Psi(p / q))^{-1}\right)=w_{N}(q / p) .
$$

Fix a convex, balanced polygon $N \subset \mathbb{R}^{2}$ whose vertices lie in $\mathbb{Z}^{2}$. Starting from a fixed vertex $v_{1}$ of $N$ and passing around $N$ in a counterclockwise fashion, we may order the remaining vertices $v_{2}, v_{3}, \ldots, v_{t}, v_{1}^{*}$, $v_{2}^{*}, \ldots, v_{t}^{*}$ where $v_{j}$ and $v_{j}^{*}$ are antipodes. The plane is decomposed into a collection of sectors by the lines based at the origin whose slopes are those of the edges of $N$. The sectors are numbered in a natural fashion $S_{1}, S_{2}, \ldots, S_{t}, S_{1}^{*}, S_{2}^{*}, \ldots, S_{t}^{*}$ as indicated in the figure below. Note that for each $j, S_{j}$ and $S_{j}^{*}$ are antipodal sectors.
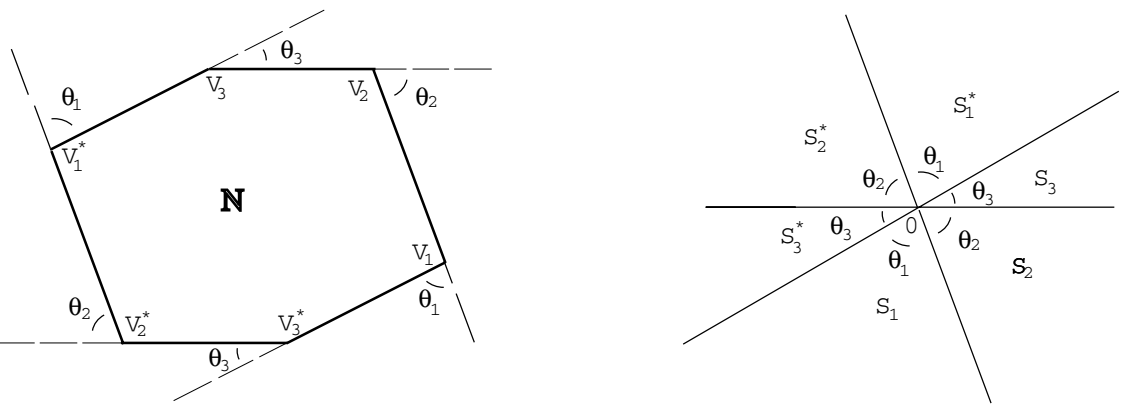

Figure 1: Sectors of a balanced polygon.

Lemma 7.2. Let $q / p$ be the slope of a line based at the origin which lies in $S_{j} \cup S_{j}^{*}$. If $v_{j}=(m, n)$ and $v_{j}^{*}=\left(m^{*}, n^{*}\right)$, then

$$
w_{N}(q / p)=\left|\left(m-m^{*}\right) q-\left(n-n^{*}\right) p\right| .
$$

Proof. Let $\left[v_{j}, v_{j}^{*}\right]$ denote the line segment in the plane spanned by $v_{j}$ and $v_{j}^{*}$. By our choice of $q / p$, if $w_{N}(q / p)=k$, then the number of lines in the plane of slope $q / p$ which contain points of both $\mathbb{Z}^{2}$ and $\left[v_{j}, v_{j}^{*}\right]$ is $k+1$. Choose integers $s, t$ such that $q s-p t=1$ and define $\Psi=\left(\begin{array}{cc}q & -p \\ -t & s\end{array}\right) \in$ $S L_{2}(\mathbb{Z})$. Then from Lemma 7.1 we see that $w_{N}(q / p)=w_{\Psi(N)}(\infty)$, while $w_{\Psi(N)}(\infty)$ is easily seen to be the absolute value of the $u$-coordinate of $\Psi(m, n)-\Psi\left(m^{*}, n^{*}\right)=\left(\left(m-m^{*}\right) q-\left(n-n^{*}\right) p,-\left(m-m^{*}\right) t+\left(n-n^{*}\right) s\right)$. 
Thus

$$
w_{N}(q / p)=\left|\left(m-m^{*}\right) q-\left(n-n^{*}\right) p\right| .
$$

q.e.d.

In our next result we obtain a closed expression for width in terms of the coordinates of the vertices of $N$.

Lemma 7.3. Let $N$ be a convex, balanced polygon whose vertices lie in $\mathbb{Z}^{2}$ and are numbered consecutively $v_{1}, \ldots, v_{t}, v_{1}^{*}, \ldots, v_{t}^{*}$ as above. Denote by $\left(m_{j}, n_{j}\right)$ the coordinates of $v_{j}$ and by $\left(m_{t+1}, n_{t+1}\right)$ those of $v_{1}^{*}$. Then for each pair of relative prime integers $p, q$,

$$
w_{N}(q / p)=\sum_{j=1}^{t}\left|\left(m_{j+1}-m_{j}\right) q-\left(n_{j+1}-n_{j}\right) p\right|
$$

Proof. Recall the sectors $S_{1}, \ldots, S_{t}, S_{1}^{*}, \ldots, S_{t}^{*}$ determined by $N$ and the given ordering of its vertices. Now the ordering of the vertices of $N$ depends only on the choice of $v_{1}$ and since $N$ is balanced, the sum $\sum_{j=1}^{t}\left|\left(m_{j+1}-m_{j}\right) q-\left(n_{j+1}-n_{j}\right) p\right|$ remains unchanged if we make any other choice. Thus we may assume that $(p, q) \in S_{1}$. Set $\alpha_{j}=$ $\left(m_{j+1}-m_{j}, n_{j+1}-n_{j}\right)$ and observe that by Lemma 7.2

$$
\begin{aligned}
w_{N}(q / p) & =\left|\left(m_{t+1}-m_{1}\right) q-\left(n_{t+1}-n_{1}\right) p\right| \\
& =\left|\sum_{j=1}^{t}\left\{\left(m_{j+1}-m_{j}\right) q-\left(n_{j+1}-n_{j}\right) p\right\}\right| \\
& =\left|\sum_{j=1}^{t} \alpha_{j} \cdot(q,-p)\right|
\end{aligned}
$$

where $\alpha_{j} \cdot(q,-p)$ denotes the scalar product between $\alpha_{j}$ and $(q,-p)$. Now by construction $\alpha_{1}, \alpha_{2}, \ldots, \alpha_{t}$ all lie in the sector $S_{2}^{*} \cup S_{3}^{*} \cup \cdots \cup S_{t}^{*}$. On the other hand, since $(q,-p)$ is obtained by rotating $(p, q) \in S_{1}$ by an angle of $\pi / 2$ in a counterclockwise direction, it lies in the sector disjoint from $\left(S_{2}^{*} \cup S_{3}^{*} \cup \cdots \cup S_{t}^{*}\right) \backslash\{(0,0)\}$ and bounded by the half-rays throuh the vectors obtained by rotating $\alpha_{1}$ clockwise by $\pi / 2$ and $\alpha_{t}$ counterclockwise by $\pi / 2$ (Figure 2 ). In particular $\alpha_{j} \cdot(q,-p) \leq 0$ for 
each $j \in\{1,2, \ldots, t\}$. Thus

$$
\begin{aligned}
w_{N}(q / p) & =\left|\sum_{j=1}^{t} \alpha_{j} \cdot(q,-p)\right| \\
& =\sum_{j=1}^{t}\left|\alpha_{j} \cdot(q,-p)\right| \\
& =\sum_{j=1}^{t}\left|\left(m_{j+1}-m_{j}\right) q-\left(n_{j+1}-n_{j}\right) p\right|
\end{aligned}
$$

as claimed.

q.e.d.

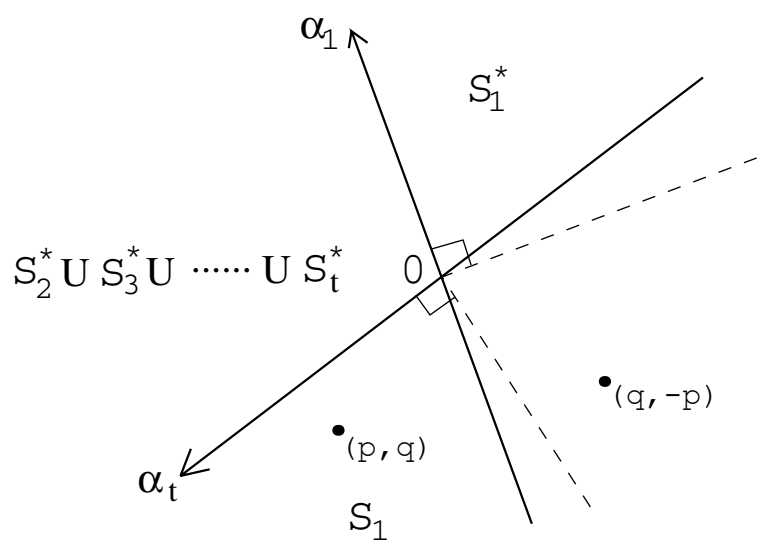

Figure 2: Calculating the width of $q / p$ from sectors.

Proposition 7.4. Let $N$ be a convex, balanced polygon whose vertices lie in $\mathbb{Z}^{2}$. Then $N$ determines a norm $\|\cdot\|_{N}: \mathbb{R}^{2} \rightarrow[0, \infty)$ satisfying

$$
\|(p, q)\|_{N}=w_{N}(q / p)
$$

for each pair of coprime integers $p, q$. Further, if the vertices of $N$ are numbered in counterclockwise order $v_{1}, v_{2}, \ldots, v_{t}, v_{1}^{*}, v_{2}^{*}, \ldots, v_{t}^{*}$ and $\left(m_{j}, n_{j}\right)$ are the coordinates of $v_{j}$ and $\left(m_{t+1}, n_{t+1}\right)$ those of $v_{1}^{*}$, then

$$
\|(u, v)\|_{N}=\sum_{j=1}^{t}\left|\left(m_{j+1}-m_{j}\right) v-\left(n_{j+1}-n_{j}\right) u\right| .
$$


Proof. According to the previous lemma, for any pair of relatively prime integers $p, q$ we have

$$
w_{N}(q / p)=\sum_{j=1}^{t}\left|\left(m_{j+1}-m_{j}\right) q-\left(n_{j+1}-n_{j}\right) p\right| .
$$

Define

$$
\|\cdot\|_{N}: \mathbb{R}^{2} \rightarrow[0, \infty)
$$

by

$$
\|(u, v)\|_{N}=\sum_{j=1}^{t}\left|\left(m_{j+1}-m_{j}\right) v-\left(n_{j+1}-n_{j}\right) u\right| .
$$

Then $\|\cdot\|_{N}$ is clearly a norm and has all the required properties. q.e.d.

Two convex, balanced polygons in $\mathbb{R}^{2}$ are called dual if each edge of one polygon is parallel to the line segment between some pair of antipodal vertices of the other.
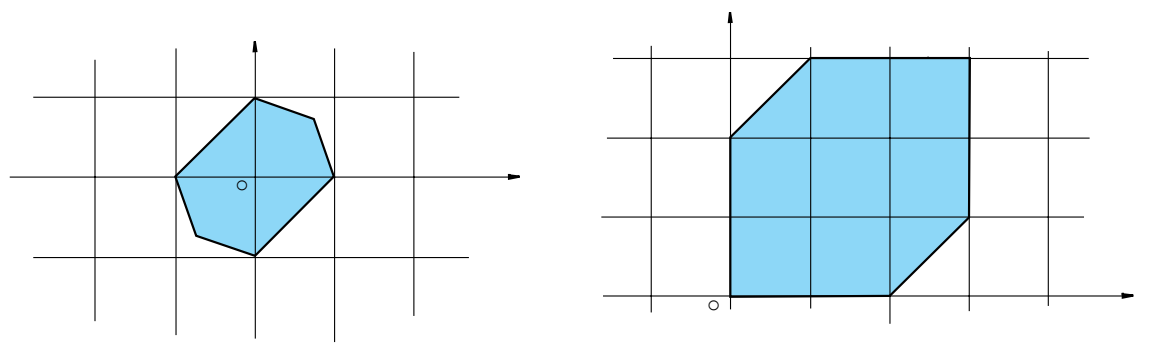

Figure 3: A pair of dual polygons.

Corollary 7.5. The boundary of a $\|\cdot\|_{N}$-ball is a convex, balanced polygon which is dual to $N$.

Proof. It follows from Lemmas 7.2 and 7.3 that

$$
\|(u, v)\|_{N}=\left|\left(m_{j}-m_{j}^{*}\right) v-\left(n_{j}-n_{j}^{*}\right) u\right| \text { for all }(u, v) \in S_{j} \cup S_{j}^{*} .
$$

In particular if $B_{1}$ is the ball of radius 1 of $\|\cdot\|_{N}$, then $\partial B_{1} \cap S_{j}$ and $\partial B_{1} \cap S_{j}^{*}$ are line segments of slope $\frac{n_{j}-n_{j}^{*}}{m_{j}-m_{j}^{*}}$. It follows that $\partial B_{1}$ is a balanced, convex polygon whose edges are parallel to the line segments in the plane whose endpoints are antipodal vertices of $N$ and whose vertices lie on the half-rays $\partial S_{1} \cup \partial S_{2} \cup \cdots \cup \partial S_{t} \cup \partial S_{1}^{*} \cup \cdots \cup \partial S_{t}^{*}$. By 
construction, the boundaries of these half-rays are parallel to the edges of $N$. Thus $N$ and $B_{1}$ are dual polygons.

q.e.d.

\section{Relations between Culler-Shalen norms and $A$-polynomials}

One of our main goals in this section is to show that the norm polygon defined by a curve in $X(M)$ and the Newton polygon of the associated $A$-polynomial are dual in the sense of the previous section. We shall continue to use the notation developed previously in the paper: for a norm curve component $X_{1}$ of $X(M),\|\cdot\|_{1}$ will denote the Culler-Shalen norm it defines, $B_{1}$ the $\|\cdot\|_{1}$-ball of radius $s_{1}=$ $\min \left\{\|\delta\|_{1} \mid \delta \in L \backslash\{0\}\right\}, D_{1}$ the plane algebraic curve associated to $X_{1}, p_{1}(u, v)$ a polynomial without repeated factors which defines $D_{1}, d_{1}$ the degree of the restriction-induced map $X_{1} \rightarrow i^{*}\left(X_{1}\right) \subset X(\partial M)$, and $A_{1}(u, v)=\left[p_{1}(u, v)\right]^{d_{1}}$ the $A$-polynomial associated to $X_{1}$ relative to the basis $\{\mu, \lambda\}$. Recall that $A_{1}(u, v)$ is well-defined up to multiplication by a nonzero complex constant and is divisible by neither $u$ nor $v$.

If the ordered basis $\{\mu, \lambda\}$ of $\pi_{1}(\partial M)$ is replaced by another $\{\sigma=$ $\left.\mu^{p} \lambda^{q}, \zeta=\mu^{s} \lambda^{t}\right\}$, where $\Psi=\left(\begin{array}{cc}p & q \\ s & t\end{array}\right) \in S L_{2}(\mathbb{Z})$, and $\hat{A}_{1}(w, z)$ is the $A$-polynomial of $X_{1}$ with respect to $\{\sigma, \zeta\}$, then there are integers $a, b$ for which

$$
\hat{A}_{1}(w, z)=w^{a} z^{b} A_{1}\left(w^{t} z^{-q}, w^{-s} z^{p}\right) .
$$

Hence we deduce the following lemma.

Lemma 8.1. Consider a new basis $\{\sigma, \zeta\}=\left\{\mu^{p} \lambda^{q}, \mu^{s} \lambda^{t}\right\}$ of $\pi_{1}(\partial M)$ where $\Psi=\left(\begin{array}{ll}p & q \\ s & t\end{array}\right) \in S L_{2}(\mathbb{Z})$. Let $N_{1}$ be the Newton polygon of the A-polynomial of $X_{1}$ relative to $\{\mu, \lambda\}$ and $\hat{N}_{1}$ the Newton polygon of the A-polynomial of $X_{1}$ relative to the basis $\left\{\mu^{p} \lambda^{q}, \mu^{s} \lambda^{t}\right\}$. Then up to a translation in the plane,

$$
\hat{N}_{1}=\left(\Psi^{\text {transpose }}\right)^{-1}\left(N_{1}\right) .
$$

Proposition 8.2. Let $X_{1}$ be a norm curve component of $X(M)$. Fix an ordered basis $\{\mu, \lambda\}$ of $H_{1}(\partial M ; \mathbb{Z}) \subset H_{1}(\partial M ; \mathbb{R})$ and let $A_{1}(u, v)$ be the A-polynomial of $X_{1}$ relative to it. If $N_{1}$ is the Newton polygon of $A_{1}(u, v)$ and $(p, q) \in L$ is a primitive class, then

$$
\|(p, q)\|_{1}=2 w_{N_{1}}(q / p) .
$$


Hence for each $(u, v) \in \mathbb{R}^{2}$,

$$
\|(u, v)\|_{1}=2\|(u, v)\|_{N_{1}} .
$$

Proof. Since $A_{1}$ is not divisible by $u$, Proposition 6.6 implies that $\|\lambda\|_{1}=2 w_{N_{1}}(\infty)$. Similarly $\|\mu\|_{1}=2 w_{N_{1}}(0)$.

Consider a pair of relatively prime integers $p, q$. Choose $s, t \in \mathbb{Z}$ such that $p t-q s=1$, set $\Psi=\left(\begin{array}{ll}q & -p \\ t & -s\end{array}\right) \in S L_{2}(\mathbb{Z})$ and observe that $\Psi(p, q)=(0,1)$. Then from Lemma 7.1 we see that $w_{N_{1}}(q / p)=$ $w_{\Psi\left(N_{1}\right)}(\infty)$. From Lemma 8.1 we see that up to a translation in the plane, $\Psi\left(N_{1}\right)$ is the Newton polygon of $X_{1}$ relative to the basis $\left\{\mu^{-s} \lambda^{-t}, \mu^{p} \lambda^{q}\right\}$ of $\pi_{1}(\partial M)$. Hence from the previous paragraph we see that $2 w_{\Psi\left(N_{1}\right)}(\infty)=\|p \mu+q \lambda\|_{1}$. The proof is completed by noting that any two norms on $\mathbb{R}^{2}$ which agree on $\mathbb{Z}^{2}$ are identical. q.e.d.

Corollary 8.3. Under the hypotheses of Proposition 8.2, the Newton polygon $N_{1}$ of $A_{1}$ and the norm polygon $B_{1}$ of $\|\cdot\|_{1}$ are dual polygons.

Proof. The conclusion follows from Proposition 8.2 and Corollary 7.5. q.e.d.

Let $X_{1}$ be a norm curve component of $X(M)$. It is shown in [11, Lemma 1.4.1] that if $x$ is an ideal point of $\widetilde{X}_{1}$, then one of the following holds:

(1) $\Pi_{x}\left(\widetilde{f}_{r}\right)=0$ for each slope $r$ on $\partial M$.

(2) There is a unique slope $r$ for which $\Pi_{x}\left(\widetilde{f}_{r}\right)=0$.

We say that a slope $r$ is associated to an ideal point $x$ of $\widetilde{X}_{1}$ (and vice versa) if $\widetilde{f}_{r}(x)$ is finite while $\widetilde{f}_{r^{\prime}}(x)=\infty$ for some other slope $r^{\prime}$. By [11], a slope $r$ associated to an ideal point of $X_{1}$ must be a boundary slope. Also note that there may be several ideal points of $X_{1}$ associated to a given slope $r$.

Corollary 8.4 ([8]). There is an edge of slope $q / p$ of the Newton polygon $N_{1}$ of the A-polynomial of $X_{1}$ if and only if $p \mu+q \lambda$ is a boundary class associated to some ideal point of $X_{1}$.

Proof. A primitive pair $\pm(p \mu+q \lambda)$ corresponds to a slope associated to some ideal point of $X_{1}$ if and only if it is a rational multiple of some vertex of the norm polygon $B_{1}$ of $\|\cdot\|_{1}$ ([2, Lemma 6.1$\left.]\right)$. Since $B_{1}$ is dual to the Newton polygon $N_{1}$, the latter occurs if and only if $N_{1}$ has an edge of slope $q / p$.

q.e.d. 
Our next result gives another characterization of $\|\cdot\|_{1}$ in terms of $A_{1}$. Define the span of a Laurent polynomial $p(z) \in \mathbb{C}\left[z, z^{-1}\right]$ to be

$$
\operatorname{span}(p)=\max \operatorname{degree}_{z} p(z)-\min \operatorname{degree}_{z} p(z) .
$$

Proposition 8.5. Suppose that $p \mu+q \lambda$ is not a boundary class associated to an ideal point of $X_{1}$. Then

$$
\|p \mu+q \lambda\|_{1}=2 \operatorname{span} A_{1}\left(z^{-q}, z^{p}\right) .
$$

Proof. The case where $p \mu+q \lambda$ equals $\mu$ or $\lambda$ follows from Corollary 6.7. For the general case fix a basis $\{\sigma, \zeta\}=\left\{\mu^{p} \lambda^{q}, \mu^{s} \lambda^{t}\right\}$ of $\pi_{1}(\partial M)$ where $p t-q s=1$. We noted before Lemma 8.1 that if $\hat{A}_{1}(w, z)$ is the $A$-polynomial of $X_{1}$ with respect to $\{\sigma, \zeta\}$, then there are integers $a, b$ for which

$$
\hat{A}_{1}(w, z)=w^{a} z^{b} A_{1}\left(w^{t} z^{-q}, w^{-s} z^{p}\right) .
$$

Since $p \mu+q \lambda$ is not a boundary class associated to an ideal point of $X_{1}$, Corollary 6.7 implies that $\|p \mu+q \lambda\|_{1}$ is twice the degree of $\hat{A}_{1}(1, z)=$ $z^{b} A_{1}\left(z^{-q}, z^{p}\right)$. Thus $\|p \mu+q \lambda\|_{1}=2 \operatorname{span} A_{1}\left(z^{-q}, z^{p}\right)$.

It is shown in $[11, \S 1.4]$ that for any slope $r$, there is a linear function $\phi_{r}: H_{1}(\partial M ; \mathbb{R}) \rightarrow \mathbb{R}$ such that

$$
\left|\phi_{r}(\delta)\right|=\sum_{\substack{\text { ideal points } x \text { of } \tilde{X}_{1} \\ \text { associated to } r}} \Pi_{x}\left(\widetilde{f}_{\delta}\right)
$$

for each $\delta \in H_{1}(\partial M ; \mathbb{Z})$. It follows that for each $\eta \in H_{1}(\partial M ; \mathbb{R})$ we have

$$
\|\eta\|_{1}=\sum_{\text {slopes } r}\left|\phi_{r}(\eta)\right|
$$

Our next result shows how the Newton polygon determines $\left|\phi_{r}\right|$.

Proposition 8.6. Suppose that $p$ and $q$ are coprime integers such that $q / p$ is the slope of an edge of the Newton polygon $N_{1}$ of $A_{1}(u, v)$. Suppose further that the endpoints of this edge have coordinates $(m, n)$ and $\left(m^{\prime}, n^{\prime}\right)$. If $r$ denotes the slope associated to $\pm(p \mu+q \lambda)$, then

$$
\left|\phi_{r}(u, v)\right|=2\left|\left(m-m^{\prime}\right) v-\left(n-n^{\prime}\right) u\right| .
$$

Proof. Let $v_{1}, v_{2}, \ldots, v_{t}, v_{1}^{*}, v_{2}^{*}, \ldots, v_{t}^{*}$ be an ordering of the vertices of $N_{1}$, as in the previous section. Let $\left(m_{j}, n_{j}\right)$ be the coordinates of $v_{j}$ 
and $\left(m_{t+1}, n_{t+1}\right)$ those of $v_{1}^{*}$. Choose relatively prime pairs of integers $\left(p_{1}, q_{1}\right),\left(p_{2}, q_{2}\right), \ldots,\left(p_{t}, q_{t}\right)$ such that $\left(m_{j+1}-m_{j}, n_{j+1}-n_{j}\right)$ is an integral multiple of $\left(p_{j}, q_{j}\right)$, say

$$
\left(m_{j+1}-m_{j}, n_{j+1}-n_{j}\right)=l_{j}\left(p_{j}, q_{j}\right) .
$$

Then by Corollary 8.4, the slopes associated with the ideal points of $X_{1}$ are $r_{1}, r_{2}, \ldots, r_{t}$ where $r_{j}$ corresponds to $\pm\left(p_{j} \mu+q_{j} \lambda\right)$. Since $\phi_{r_{j}}\left(p_{j}, q_{j}\right)$ $=0$, there is a nonzero integer $l_{j}^{\prime}$ for which

$$
\phi_{r_{j}}(u, v)=\left|l_{j}^{\prime}\right|\left|p_{j} v-q_{j} u\right| .
$$

Thus if $(u, v) \in \mathbb{R}^{2} \equiv V$,

$$
\begin{aligned}
& \sum_{j=1}^{t}\left|l_{j}^{\prime}\right|\left|p_{j} v-q_{j} u\right| \\
& =\sum_{j=1}^{t}\left|\phi_{r_{j}}(u, v)\right| \\
& =\|(u, v)\|_{1} \\
& =2\|(u, v)\|_{N_{1}} \text { by Proposition } 8.2 \\
& =\sum_{j=1}^{t} 2\left|\left(m_{j+1}-m_{j}\right) v-\left(n_{j+1}-n_{j}\right) u\right| \text { by the definition of }\|\cdot\|_{N_{1}} \\
& =\sum_{j=1}^{t} 2\left|l_{j} \| p_{j} v-q_{j} u\right| .
\end{aligned}
$$

Thus for each $(u, v) \in \mathbb{R}^{2}, \sum_{j=1}^{t}\left(2\left|l_{j}\right|-\left|l_{j}^{\prime}\right|\right)\left|p_{j} v-q_{j} u\right|=0$. Since $\left(p_{j}, q_{j}\right)$ and $\left(p_{k}, q_{k}\right)$ are linearly independent for $j \neq k$, it follows that for each value of the index $j$,

$$
\begin{aligned}
\phi_{r_{j}}(u, v)=\left|l_{j}^{\prime}\right|\left|p_{j} v-q_{j} u\right| & =2\left|l_{j}\right|\left|p_{j} v-q_{j} u\right| \\
& =2\left|\left(m_{j+1}-m_{j}\right) v-\left(n_{j+1}-n_{j}\right) u\right|
\end{aligned}
$$

as claimed. This completes the proof.

q.e.d.

Next we extend the results of this section to more general curves in $X(M)$. Consider $C=X_{1} \cup \ldots \cup X_{k} \subset X(M)$ where each $X_{j}$ is a norm curve component of $X(M)$. Recall, from $\S 6$ that the $A$ polynomial of $C$, with respect to a basis $\{\mu, \lambda\}$ of $\pi_{1}(\partial M)$, is given by $A_{\mathrm{C}}(u, v)=\Pi_{j=1}^{k} A_{j}(u, v)$, where $A_{j}$ is the $A$-polynomial of $X_{j}$ with respect to $\{\mu, \lambda\}$. Let $N_{\mathrm{C}}, N_{1}, \ldots, N_{k}$ be the associated Newton polygons and $w_{N_{\mathrm{C}}}, w_{N_{1}}, \ldots, w_{N_{k}}$ their width functions. 
Lemma 8.7. $w_{N_{C}}=w_{N_{1}}+w_{N_{2}}+\cdots+w_{N_{k}}$.

Proof. Since $w_{N_{\mathrm{C}}}(0)$ is the largest power of $v$ occurring in $A_{\mathrm{C}}(u, v)$, and similarly for each $w_{N_{j}}(0)$, it is clear that $w_{N_{\mathrm{C}}}(0)=w_{N_{1}}(0)+w_{N_{2}}(0)+$ $\cdots+w_{N_{k}}(0)$.

Let $p, q$ be an arbitrary pair of coprime integers and choose $s, t \in \mathbb{Z}$ such that $t p-s q=1$. Set $\Psi=\left(\begin{array}{cc}t & -s \\ -q & p\end{array}\right)$ and observe that by Lemma 7.1 we have $w_{N_{\mathrm{C}}}(q / p)=w_{\Psi\left(N_{\mathrm{C}}\right)}(0)$. Now $\Psi\left(N_{\mathrm{C}}\right)$ is the Newton polygon of the Laurent polynomial

$$
\begin{aligned}
\hat{A}_{\mathrm{C}}(w, z) & =A_{\mathrm{C}}\left(w^{p} z^{q}, w^{s} z^{t}\right) \\
& =A_{1}\left(w^{p} z^{q}, w^{s} z^{t}\right) A_{2}\left(w^{p} z^{q}, w^{s} z^{t}\right) \ldots A_{k}\left(w^{p} z^{q}, w^{s} z^{t}\right) \\
& =\hat{A}_{1}(w, z) \hat{A}_{2}(w, z) \ldots \hat{A}_{k}(w, z) .
\end{aligned}
$$

Hence if $\hat{N}_{\mathrm{C}}, \hat{N}_{j}$ are the Newton polygons of $\hat{A}_{\mathrm{C}}, \hat{A}_{j}, j=1, \ldots, k$, then $\hat{N}_{\mathrm{C}}=\Psi\left(N_{\mathrm{C}}\right)$ and $\hat{N}_{j}=\Psi\left(N_{j}\right)$. Thus

$$
\begin{aligned}
w_{N_{\mathrm{C}}}(q / p) & =w_{\Psi\left(N_{\mathrm{C}}\right.}(0) \\
& =w_{\hat{N}_{\mathrm{C}}}(0) \\
& =w_{\hat{N}_{1}}(0)+w_{\hat{N}_{2}}(0)+\cdots+w_{\hat{N}_{k}}(0) \\
& =w_{\Psi\left(N_{1}\right)}(0)+w_{\Psi\left(N_{2}\right)}(0)+\cdots+w_{\Psi\left(N_{k}\right)}(0) \\
& =w_{N_{1}}(q / p)+w_{N_{2}}(q / p)+\cdots+w_{N_{k}}(q / p) .
\end{aligned}
$$

This completes the proof.

q.e.d.

Theorem 8.8. Suppose that $X_{1}, X_{2}, \ldots, X_{k}$ is a collection of distinct norm curve components of $X(M)$ and set $C=X_{1} \cup X_{2} \cup \cdots \cup X_{k}$. Let $\|\cdot\|_{C}$ be the Culler-Shalen norm of $C$ and $\|\cdot\|_{N_{C}}$ the width function norm determined by the Newton polygon $N_{C}$ of the A-polynomial of $C$. Then the following results hold:

(1) $2\|\cdot\|_{N_{C}}=\|\cdot\|_{C}$.

(2) If $p \mu+q \lambda$ is not a boundary class associated to an ideal point of $C$, then

$$
\|p \mu+q \lambda\|_{C}=\operatorname{span} A_{C}\left(z^{q}, z^{-p}\right) .
$$

(3) $N_{C}$ is balanced and dual to the norm polygon $B_{C}$ of $\|\cdot\|_{C}$.

(4) $N_{C}$ has an edge of slope $q / p$ if and only if $B_{C}$ has a vertex whose associated slope is $q / p$. 
(5) Suppose that $r$ is a slope on $\partial M$ and $\phi_{r}$ the linear functional on $H_{1}(\partial M ; \mathbb{R})$ which satisfies

$$
\left|\phi_{r}(\alpha)\right|=\sum_{j=1}^{k}\left(\sum_{\substack{\text { ideal points } x \text { of } \tilde{X}_{j} \\ \text { associated to } r}} \Pi_{x}\left(f_{\alpha}\right)\right)
$$

for $\alpha \in H_{1}(\partial M ; \mathbb{Z})$. If $(m, n)$ and $\left(m^{\prime}, n^{\prime}\right)$ are the coordinates of the endpoints of an edge of $N$ of slope $q / p$, then

$$
\left|\phi_{r}(u, v)\right|=2\left|\left(m-m^{\prime}\right) v-\left(n-n^{\prime}\right) u\right| .
$$

Proof. Let $\|\cdot\|_{j}$ be the Culler-Shalen norm of $X_{j}$ and $N_{j}$ the Newton polygon of its $A$-polynomial.

If $p, q$ are relatively prime integers, then

$$
\begin{aligned}
2 \|(p, q)) \|_{N_{\mathrm{C}}} & =2 w_{N_{\mathrm{C}}}(q / p) \\
& =2 \sum_{j=1}^{k} w_{N_{j}}(q / p) \quad \text { by Lemma } 8.7 \\
& =2 \sum_{j=1}^{k}\|(p, q)\|_{N_{j}} \\
& =\sum_{j=1}^{k}\|(p, q)\|_{j} \quad \text { by Proposition } 8.2 \\
& =\|(p, q)\|_{\mathrm{C}} .
\end{aligned}
$$

Hence (1) holds.

Next observe that part (2) is a consequence of the definitions and Proposition 8.5, while part (3) follows from (1), Corollary 7.5 and Corollary 6.3. Finally (4) and (5) are proven exactly as the analogous results are handled in the proofs of Corollary 8.4 and Proposition 8.6. q.e.d.

\section{The canonical curve and norm}

In this section we define the canonical curve in the character variety of a hyperbolic knot exterior and study the associated norm and 
$A$-polynomial. We shall continue to use the notations established in previous sections.

According to the rigidity theorem for hyperbolic 3-manifolds of finite volume $[34, \S 5]$, there are exactly two conjugacy classes of discrete faithful representations of $\pi_{1}(M)$ in $P S L_{2}(\mathbb{C})$. Further the characters of these two conjugacy classes differ by complex conjugation. Thurston proved that all such representations lift to $S L_{2}(\mathbb{C})$ (see $[12$, Proposition 3.1.1]), and thus the set of characters of such lifts consists of two orbits of the $H^{1}\left(M ; \mathbb{Z}_{2}\right)$-action on $X(M)$, one orbit the complex conjugate of the other. It can then be shown that there are precisely $2\left|H^{1}\left(M ; \mathbb{Z}_{2}\right)\right|=2\left|H_{1}\left(M ; \mathbb{Z}_{2}\right)\right|$ conjugacy classes of discrete faithful representations of $\pi_{1}(M)$ in $S L(2, \mathbb{C})$ ([26, Corollary V.1.3], compare Lemma 9.5).

Let $X_{1}, X_{2}, \ldots, X_{k}$ be the components of $X(M)$ which contain the character of a discrete faithful representation and recall from $\S 4$ that each is a norm curve component. From the discussion above we see that $C_{M}$, the $\operatorname{Aut}(\mathbb{C}) \times H_{1}\left(M ; \mathbb{Z}_{2}\right)$-orbit of $X_{1}(\mathrm{cf}, \S 5)$, contains $X_{2}, \ldots, X_{k}$. Indeed it characterized by the fact that it is the smallest subvariety of $X(M)$ which is defined over the rationals and which contains these curves. Thus we call $C_{\mathrm{M}}$ the canonical norm curve of $X(M)$.

Suppose that $C_{M}$ contains $n$ algebraic components, say

$$
C_{M}=X_{1} \cup X_{2} \cup \ldots X_{n}
$$

By Lemmas 5.4 and 5.3 each $X_{j}$ is a norm curve component of $X(M)$. Let $\|\cdot\|_{j}, s_{j}$, and $B_{j}$ denote respectively the Culler-Shalen norm, the minimal nonzero value of $\|\cdot\|_{j}$, and the $\|\cdot\|_{j}$-ball of radius $s_{j}$. Let $\|\cdot\|_{M}$, $s_{M}$, and $B_{M}$ denote those of $C_{M}$. We call $\|\cdot\|_{M}$ the canonical norm on $H_{1}(M ; \mathbb{Z})$.

\section{Proposition 9.1.}

(1) $\|\cdot\|_{M}=n\|\cdot\|_{1}, s_{M}=n s_{1}$, and $B_{M}=B_{1}$.

(2) $B_{M}$ is a finite-sided convex polygon balanced about the origin whose vertices are rational multiples of strict boundary classes of $L$.

(3) $B_{M}$ contains at most three (pairs of) nontrivial classes of $L$ which are not vertices of $B_{M}$, and their mutual distances are at most one.

(4) Choose a basis $\{\mu, \lambda\}$ for $L$ such that $\|\mu\|_{M}=s_{M}$ and identify $H_{1}(\partial M ; \mathbb{R})$ with $\mu \lambda$-plane. If $(a, b) \in B_{M}$, then $|b| \leq 2$. Further if 
there is some $(a, b) \in B_{M}$ with $b=2$, then $(a, b) \in L$ and $B_{M}$ is a parallelogram with vertices $\pm(1,0)$ and $\pm(a, b)$.

Proof. Part (1) is a consequence of the definition of $\|\cdot\|_{M}$ and the Lemmas 5.4 and 5.3. The rest follows mostly from Proposition 4.5, though for the strictness of the boundary slopes in part (2) we combine Lemma 5.3 with the observation that discrete faithful representations are not virtually reducible.

q.e.d.

Lemma 9.2. The inequality $4 \leq 2\left|H_{1}\left(M ; \mathbb{Z}_{2}\right)\right| \leq s_{M}$ holds.

Proof. We noted above that there are exactly $2\left|H_{1}\left(M ; \mathbb{Z}_{2}\right)\right|$ characters of discrete faithful representations of $\pi_{1}(M)$ in $S L(2, \mathbb{C})$, and each of these characters lies in $C_{M}$. Fix $\mu \in L \cap \partial B_{M}$ and note that since $f_{\mu}$ takes the value zero at each of these characters, we have $s_{\mathrm{M}}=\|\mu\|_{\mathrm{M}}=$ $\left.\sum_{j=1}^{n} \operatorname{degree}\left(f_{\mu}\right)\right|_{\tilde{X}_{j}} \geq 2\left|H_{1}\left(M ; \mathbb{Z}_{2}\right)\right|$ q.e.d.

The inequality given in the lemma is sharp as $s_{\mathrm{M}}=4$ when $M$ is the figure eight knot exterior. For this manifold, $C_{M}$ is an irreducible curve (see [6] for details).

Proposition 9.3. Fix a basis $\{\mu, \lambda\}$ of $L$ where $\|\mu\|_{M}=s_{M}$ and identify $H_{1}(\partial M ; \mathbb{R})$ with the $\mu \lambda$-plane. Suppose that $\alpha \in L$ is a finite or cyclic filling class but is not a strict boundary class.

(1) If $\alpha$ is a C-type filling class, then:

(i) $\|\alpha\|_{M}=s_{M}$. Hence $\alpha \in \partial B_{M}$ but is not a vertex of $B_{M}$.

(ii) The absolute value of the $\lambda$-coordinate of $\alpha$ is less than or equal to 1.

(2) If $\alpha$ is a D-type or a $Q$-type filling class, then:

(i) $\|\alpha\|_{M} \leq 2 s_{M}$.

(ii) $\|\alpha\|_{M} \leq\|\beta\|_{M}$ for any nontrivial element $\beta \in L$ satisfying $\Delta(\alpha, \beta) \equiv 0(\bmod 2)$.

(iii) The absolute value of the $\lambda$-coordinate of $\alpha$ is less than or equal to 1 .

(3) (a) If $\alpha$ is a $T(q)$-type filling class and $H_{1}(M ; \mathbb{Z})$ has no 3 torsion, then $q=3$ and:

(i) $\|\alpha\|_{M} \leq s_{M}+4$. 
(ii) $\|\alpha\|_{M} \leq\|\beta\|_{M}$ for any nontrivial class $\beta \in L$ satisfying $\Delta(\alpha, \beta) \equiv 0(\bmod q)$.

(iii) If $\beta \in L$ is a finite filling class satisfying $\Delta(\alpha, \beta) \equiv 0$ $(\bmod q)$, then $\beta$ is also of type $T(q)$ and $\|\beta\|_{M}=\|\alpha\|_{M}$.

(b) If $\alpha$ is a $T(q)$-type filling class and $H_{1}(M ; \mathbb{Z})$ has 3 -torsion, then $q \in\{1,2\}$ and:

(i) $\|\alpha\|_{M} \leq s_{M}+4$ and $q=2$ if $\|\alpha\|_{M}>s_{M}$.

(ii) $\|\alpha\|_{M} \leq\|\beta\|_{M}$ for any nontrivial class $\beta \in L$ satisfying $\Delta(\alpha, \beta) \equiv 0(\bmod q)$.

(iii) If $\beta \in L$ is a finite filling class satisfying $\Delta(\alpha, \beta) \equiv 0$ $(\bmod q)$, then $\beta$ is also of type $T(q)$ and $\|\beta\|_{M}=\|\alpha\|_{M}$.

(4) If $\alpha$ is an $I(q)$-type filling class, then $q \in\{1,2,3,5\}$ and:

(i) $\|\alpha\|_{M} \leq s_{M}+8$ and $q>1$ if $\|\alpha\|_{M}>s_{M}$.

(ii) $\|\alpha\|_{M} \leq\|\beta\|_{M}$ for any nontrivial class $\beta \in L$ satisfying $\Delta(\alpha, \beta)$ $\equiv 0(\bmod q)$.

(iii) If $\beta \in L$ is a finite filling class satisfying $\Delta(\alpha, \beta) \equiv 0(\bmod$ $q)$, then $\beta$ is also of type $I(q)$ and $\|\beta\|_{M}=\|\alpha\|_{M}$.

(5) (a) If $\alpha$ is an $O(q)$-type filling class and $H_{1}(M ; \mathbb{Z})$ has no 2torsion, then $q \in\{2,4\}$ and:

(i) $\|\alpha\|_{M} \leq s_{M}+6$.

(ii) $\|\alpha\|_{M} \leq\|\beta\|_{M}$ for any nontrivial class $\beta \in L$ satisfying $\Delta(\alpha, \beta) \equiv 0(\bmod q)$.

(iii) If $\beta \in L$ is a finite filling class satisfying $\Delta(\alpha, \beta) \equiv 0$ $(\bmod q)$, then $\beta$ is also of type $O(q)$ and $\|\beta\|_{M}=\|\alpha\|_{M}$.

(b) If $\alpha$ is an $O(q)$-type filling class and $H_{1}(M ; \mathbb{Z})$ has 2 -torsion, then $q \in\{1,2,3\}$ and:

(i) $\|\alpha\|_{M} \leq s_{M}+12$ and $q>1$ if $\|\alpha\|_{M}>s_{M}$.

(ii) $\|\alpha\|_{M} \leq\|\beta\|_{M}$ for any nontrivial class $\beta \in L$ satisfying $\Delta(\alpha, \beta) \equiv 0(\bmod q)$.

(iii) If $\beta \in L$ is a finite filling class satisfying $\Delta(\alpha, \beta) \equiv 0$ $(\bmod q)$, then $\beta$ is also of type $O(q)$ and $\|\beta\|_{M}=\|\alpha\|_{M}$.

Proof. Parts (1), (2) of the proposition follow directly from Proposition 4.6 (1), (2) and Proposition 9.1. Parts (3) (ii) and (iii) are consequences of Propositions 4.6 (3) and 3.3 respectively. To prove part (3) (i), first recall that

$$
\widetilde{C}_{\mathrm{M}}=\widetilde{X}_{1} \cup \cdots \cup \widetilde{X}_{n}
$$


For each $\delta \in L$,

$$
\|\delta\|_{\mathrm{M}}=\sum_{j=1}^{n}\|\delta\|_{j}=\sum_{j=1}^{n} \operatorname{degree}\left(\left.\widetilde{f}_{\delta}\right|_{\widetilde{X}_{j}}\right)=\sum_{j=1}^{n} \sum_{x \in \tilde{X}_{j}} Z_{x}\left(\widetilde{f}_{\delta}\right)
$$

where $Z_{x}\left(\widetilde{f}_{\delta}\right)$ is the multiplicity of $x$ as a zero of $\widetilde{f}_{\delta}$. Hence

$$
\|\alpha\|_{\mathrm{M}}=\|\mu\|_{\mathrm{M}}+\left(\|\alpha\|_{\mathrm{M}}-\|\mu\|_{\mathrm{M}}\right)=s_{\mathrm{M}}+\sum_{j=1}^{n} \sum_{x \in \widetilde{X}_{j}}\left(Z_{x}\left(\widetilde{f}_{\alpha}\right)-Z_{x}\left(\widetilde{f}_{\mu}\right)\right) .
$$

Thus if $J=\left\{x \in \widetilde{C}_{\mathrm{M}} \mid Z_{x}\left(\widetilde{f}_{\alpha}\right)>Z_{x}\left(\widetilde{f}_{\mu}\right)\right\}$, then

$$
\|\alpha\|_{\mathrm{M}} \leq s_{\mathrm{M}}+\sum_{x \in J}\left(Z_{x}\left(\widetilde{f}_{\alpha}\right)-Z_{x}\left(\widetilde{f}_{\mu}\right)\right)
$$

Recall that $\widetilde{X}_{j}=X_{j}^{\nu} \cup \mathcal{I}_{j}$ where $X_{j}^{\nu} \stackrel{\nu}{\rightarrow} X_{j}$ is the normalization of $X_{j}$ and $\mathcal{I}_{j}$ is the finite set of ideal points of $\widetilde{X}_{j}$. Fix $x \in J \cap \widetilde{X}_{j}$. The hypothesis that $\alpha$ is not a strict boundary slope implies that $x \in X_{j}^{\nu}$ ([11, Prop. I.6.1] and Proposition 4.5). We now proceed in the manner of $[2, \S 4]$. It was shown there that:

- $Z_{x}\left(\tilde{f}_{\alpha}\right)-Z_{x}\left(\tilde{f}_{\mu}\right)=2$.

- The Zariski tangent space of $X(M)$ at $\nu(x)$ is 1-dimensional.

- $\nu(J)$ is the set of characters in $C_{\mathrm{M}}$ correspondingto representations which send $\alpha$ to $\{ \pm I\}$ and whose image is the binary tetrahedral group.

The first statement shows that $\|\alpha\|_{\mathrm{M}} \leq s_{\mathrm{M}}+2|J|$. It follows from the second that $\nu(x)$ is a simple point of $X(M)$ (cf. $\S 2)$ and therefore $\nu^{-1}(\nu(x))=x$. Hence $\|\alpha\|_{\mathrm{M}} \leq s_{\mathrm{M}}+2|\nu(J)|$. Finally combining the third with the method of [2, Lemma 5.6] implies that $|\nu(J)|=2$. Thus $\|\alpha\|_{\mathrm{M}} \leq s_{\mathrm{M}}+4$, as claimed.

Parts (4) and (5) can be proved similarly.

Remark 9.4. The previous proposition can be sharpened under certain additional assumptions. For instance if $\mu$ is a cyclic filling class, but not a strict boundary class, then $Z_{x}\left(\widetilde{f}_{\mu}\right) \leq Z_{x}\left(\widetilde{f}_{\alpha}\right)$ for each $x \in$ 
$\widetilde{C}_{\mathrm{M}}[11$, Proposition 1.1.3]. Hence following the method of proof of Proposition 9.3 we have

$$
\|\alpha\|_{\mathrm{M}}= \begin{cases}s_{\mathrm{M}} \text { or } s_{\mathrm{M}}+4 & \text { if } \alpha \text { is } T \text {-type } \\ s_{\mathrm{M}} \text { or } s_{\mathrm{M}}+8 & \text { if } \alpha \text { is } I \text {-type } \\ s_{\mathrm{M}}, s_{\mathrm{M}}+4, s_{\mathrm{M}}+8, \text { or } s_{\mathrm{M}}+12 & \text { if } \alpha \text { is } O \text {-type } \\ & \text { and } H_{1}\left(M ; \mathbb{Z}_{2}\right)=\mathbb{Z}_{2} \oplus \mathbb{Z}_{2}\end{cases}
$$

We close this section with some useful properties concerning the canonical norm of a class in the kernel of $H_{1}\left(\partial M ; \mathbb{Z}_{2}\right) \rightarrow H_{1}\left(M ; \mathbb{Z}_{2}\right)$.

Lemma 9.5. $H^{1}\left(M ; \mathbb{Z}_{2}\right)$ acts freely on the Zariski open set of nonvirtually reducible characters in $C_{M}$.

Proof. Let $\chi_{\rho} \in C_{\mathrm{M}}$ be a non-virtually reducible character and suppose there is an $\epsilon \in H^{1}\left(M ; \mathbb{Z}_{2}\right)$ such that $\chi_{\rho}=\epsilon \cdot \chi_{\rho}$. We will show that $\epsilon$ is the trivial homomorphism $\pi_{1}(M) \rightarrow\{ \pm I\}$.

Since $\rho$ is irreducible and $\epsilon \cdot \chi_{\rho}=\chi_{\epsilon \rho}$, there is a matrix $A \in S L(2, \mathbb{C})$ satisfying $\epsilon \rho=A \rho A^{-1}$. Suppose that there is some $\xi \in \pi_{1}(M)$ for which $\epsilon(\xi)=-I$. Set $\rho(\xi)=B$. Then $-B=\epsilon(\xi) \rho(\xi)=A \rho(\xi) A^{-1}=A B A^{-1}$, and so $A=-B A B^{-1}$. It follows that $\operatorname{trace}(A)=0$, and so without loss of generality we may assume that $A=\left(\begin{array}{cc}i & 0 \\ 0 & -i\end{array}\right)$. The subgroup $\tilde{\pi}=\operatorname{ker}(\epsilon)$ of $\pi_{1}(M)$ has index 2 and for any $\gamma \in \tilde{\pi}, \rho(\gamma)=A \rho(\gamma) A^{-1}$. It follows that $\rho(\gamma)$ is diagonal and thus $\rho \mid \widetilde{\pi}$ is a reducible representation. But this contradicts our choice of $\rho$. Hence $\epsilon$ is the trivial element of $H^{1}\left(M ; \mathbb{Z}_{2}\right)$.

q.e.d.

Consider the $\left|H_{1}\left(M ; \mathbb{Z}_{2}\right)\right|$-sheeted regular covering $p: \widetilde{M} \rightarrow M$ corresponding to the surjective homomorphism $\pi_{1}(M) \rightarrow H_{1}\left(M ; \mathbb{Z}_{2}\right)$. The homomorphism $p_{\#}: \pi_{1}(\widetilde{M}) \rightarrow \pi_{1}(M)$ induces a regular map $p^{*}: X(M) \rightarrow$ $X(\widetilde{M})$ and the closure $E_{\mathrm{M}}$ of $p^{*}\left(C_{\mathrm{M}}\right)$ in $X(\widetilde{M})$ is a curve in $X(\widetilde{M})$. It is an elementary exercise to show that if $\chi_{1}, \chi_{2} \in C_{\mathrm{M}}$ are two non-virtually reducible characters then $p^{*}\left(\chi_{1}\right)=p^{*}\left(\chi_{2}\right)$ if and only if $\chi_{2}=\epsilon \chi_{1}$ for some $\epsilon \in H^{1}\left(M ; \mathbb{Z}_{2}\right)$. Hence by Proposition 4.2 and Lemma 9.5, $\widetilde{p}^{*}: \widetilde{C} \rightarrow \widetilde{E}$ is generically a $\left|H_{1}\left(M ; \mathbb{Z}_{2}\right)\right|$-to-one map.

Fix a boundary component $\widetilde{T}$ of $\partial \widetilde{M}$. For any element $\widetilde{\delta} \in \widetilde{L}=$ $H_{1}(\widetilde{T} ; \mathbb{Z}) \subset L$ (we identify $\widetilde{L}$ with $\left.p_{\#}(\widetilde{L})\right)$ let $I_{\widetilde{\delta}}: X(\widetilde{M}) \rightarrow \mathbb{C}$ be the evaluation map and set $f_{\widetilde{\delta}}=I_{\widetilde{\delta}}^{2}-4$. There is a commutative diagram 
of surjective rational maps

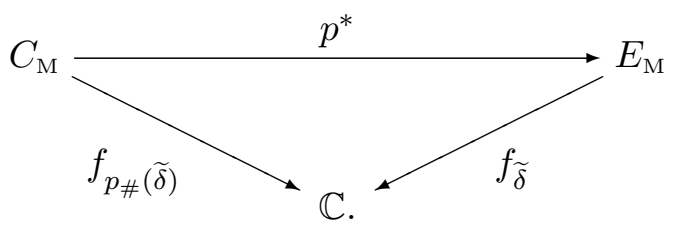

Define the degree of a rational function $f: X \rightarrow Y$ between curves to be $\sum \operatorname{degree}\left(f \mid X_{j}: X_{j} \rightarrow \overline{f\left(X_{j}\right)}\right)$, where the sum is over the algebraic components $X_{j}$ of $X$. Then

$$
\left\|p_{\#}(\widetilde{\delta})\right\|_{\mathrm{M}}=\operatorname{degree}\left(f_{p_{\#}(\widetilde{\delta})} \mid C_{\mathrm{M}}\right)=\left|H_{1}\left(M ; \mathbb{Z}_{2}\right)\right| \operatorname{degree}\left(f_{\widetilde{\delta}} \mid E_{\mathrm{M}}\right) .
$$

If $\widetilde{f}_{\widetilde{\delta}} \mid \widetilde{C}_{\mathrm{M}}$ has $p$ distinct poles, then its degree is at least $2 p$ since $f_{\widetilde{\delta}}=$ $\left(I_{\widetilde{\delta}}+2\right)\left(I_{\widetilde{\delta}}-2\right)$. In order to apply this observation, let $\beta_{1}, \beta_{2}, \ldots, \beta_{p}$ be the strict boundary classes associated to the vertices of $B_{\mathrm{M}}$. Then for any $j=1,2, \ldots, n$ there are ideal points $x_{1}, x_{2}, \ldots, x_{p}$ of $\widetilde{X}_{j}$ such that for $\delta \in L, \widetilde{f}_{\delta}\left(x_{j}\right) \in \mathbb{C}$ if and only $\delta$ is a nonzero, integral multiple of $\beta_{j}$. For each $j$, choose a class $\widetilde{\beta}_{j} \in \widetilde{L}$ such that $p_{\#}\left(\widetilde{\beta}_{j}\right)$ is a nonzero multiple of $\beta_{j}$. Then from the commutativity of the diagram above we see that $\widetilde{f}_{\widetilde{\delta}}\left(p^{*}\left(x_{j}\right)\right) \in \mathbb{C}$ if and only $p_{\#}(\widetilde{\delta})$ is a nonzero multiple of $\beta_{j}$. It follows that for any $\widetilde{\delta} \in \widetilde{L} \backslash\{0\}, \widetilde{f}_{\widetilde{\delta}}$ has at least $p-1$ distinct poles in $\widetilde{X}_{j}$. Further if $p_{*}(\widetilde{\delta})$ is a primitive class which is not a boundary class associated to a vertex of $B_{\mathrm{M}}$, then $\widetilde{f}_{\widetilde{\delta}}$ has at least $p$ distinct poles. In summary, we have derived the following proposition.

Proposition 9.6. Suppose that $\alpha \in L$ is a primitive class which lies in the kernel of the homomorphism $H_{1}\left(\partial M ; \mathbb{Z}_{2}\right) \rightarrow H_{1}\left(M ; \mathbb{Z}_{2}\right)$ induced by inclusion. Let $n$ be the number of algebraic components of $C_{M}$.

(1) $\|\alpha\|_{M}$ is divisible by $2 n\left|H_{1}\left(M ; \mathbb{Z}_{2}\right)\right|$.

(2) If $B_{M}$ has $p$ pairs of vertices then

$$
\|\alpha\|_{M} \geq \begin{cases}2 n(p-1)\left|H_{1}\left(M ; \mathbb{Z}_{2}\right)\right| & \text { if } \alpha \text { is a rational multiple } \\ & \text { of a vertex of } B_{M} \\ 2 n p\left|H_{1}\left(M ; \mathbb{Z}_{2}\right)\right| & \text { if } \alpha \text { is a rational multiple } \\ & \text { of a vertex of } B_{M} .\end{cases}
$$




\section{The canonical $A$-polynomial}

Let $X_{1}, X_{2}, \ldots, X_{n}$ be the algebraic components of $C_{\mathrm{M}}$ and $\mathcal{B}=$ $\{\mu, \lambda\}$ a basis for $\pi_{1}(\partial M)$. Suppose that $\left.A_{j}(u, v)\right)$ is the $A$-polynomial of $X_{j}$ with respect to $\mathcal{B}$. The canonical $A$-polynomial of $M$, with respect to the basis $\mathcal{B}$, is the product

$$
A_{M}(u, v)=A_{1}(u, v) A_{2}(u, v) \ldots A_{n}(u, v) .
$$

Let $N_{\mathrm{M}}$ be the associated Newton polygon. Our next results follow immediately from Theorem 8.8 and Proposition 6.10.

Proposition 10.1. The canonical norm polygon $B_{M}$ is dual to the canonical Newton polygon $N_{M}$ and $\|\cdot\|_{M}=\|\cdot\|_{N_{M}}$. Further, $\|\mu\|_{M}=2 n_{0}$ and $\|\lambda\|_{M}=2 m_{0}$, where $n_{0}$ is the maximal exponent of $v$ in $A_{M}(u, v)$ and $m_{0}$ that of $u$.

Proposition 10.2. If $p \mu+q \lambda$ is not a boundary class associated to an ideal point of $C_{M}$, then

$$
\|p \mu+q \lambda\|_{M}=2 \operatorname{span} A_{M}\left(z^{q}, z^{-p}\right) .
$$

Proposition 10.3. The canonical A-polynomial $A_{M}(u, v)=$ $\sum a_{m, n} u^{m} v^{n}$ of $M$ has the following properties:

(1) After multiplying by a nonzero complex constant, $A_{M}(u, v)$ may be taken to have integer coefficients whose greatest common denominator is 1. Such a representative is well-defined up to sign.

(2) Let $m_{0}$ be the maximal exponent of $u$ occurring in $A_{M}(u, v)$ and $n_{0}$ that of $v$. Then for some $\epsilon \in\{ \pm 1\}, a_{m, n}=\epsilon a_{m_{0}-m, n_{0}-n}$ for all $m$ and $n$.

(3) The coefficients of $A_{M}(u, v)$ indexed by the corners of $N$ are equal to \pm 1 .

(4) The nonzero roots of an edge polynomial $f_{E}(z)$ or $g_{E}(z)$ determined by $A_{M}(u, v)$ are roots of the unity. In fact $f_{E}(z)$ and $g_{E}(z)$ are products of a power of $z$ with some cyclotomic polynomials. Further if $\alpha=(p, q)$ is the boundary class associated to the edge $E$ and if \pm 1 is a root of either $f_{E}(z)$ or $g_{E}(z)$, then $f_{\alpha}$ takes the value zero at some ideal point of $\widetilde{C}_{M}$.

(5) Let $i: \partial M \rightarrow M$ be the inclusion and $i_{*}: H_{1}\left(\partial M ; \mathbb{Z}_{2}\right) \rightarrow H_{1}\left(M ; \mathbb{Z}_{2}\right)$ the associated homomorphism. 
(i) If $i_{*}(\mu) \neq 0$ and $i_{*}(\lambda)=0$, then $a_{m, n}=0$ when $m$ is odd.

(ii) If $i_{*}(\mu)=0$ and $i_{*}(\lambda) \neq 0$, then $a_{m, n}=0$ when $n$ is odd.

(iii) If $i_{*}(\mu) \neq 0$ and $i_{*}(\lambda) \neq 0$, then $a_{m, n}=0$ when $m+n$ is odd.

Corollary 10.4 ([8]). If $M$ is a knot exterior in $S^{3}$ and $\{\mu, \lambda\}$ is the standard meridian-longitude basis of $L$, then the powers of $u$ occurring in the canonical A-polynomial of $M$ are even numbers.

\section{Applications to Dehn filling}

In this section we discuss some results concerning the manifolds obtained by Dehn filling on $M$, results derived from the canonical polynomial $A_{\mathrm{M}}(u, v)$.

A 3-manifold is small if it does not contain any closed incompressible surfaces. If a small 3-manifold is homeomorphic to a Seifert fibred space, then it admits a Seifert structure whose base orbifold is $S^{2}$ with at most three singular fibres $[20, \S I V]$.

Proposition 11.1. Let $X_{1}$ be a norm curve component of $X(M)$ and suppose that $\alpha \in L$ is a primitive class. Suppose that $x \in X_{1}^{\nu}$ where $Z_{x}\left(\tilde{f}_{\alpha}\right)>Z_{x}\left(\tilde{f}_{\delta}\right)$ for some $\delta \in L \backslash\{0\}$ and $\nu(x)=\chi_{\rho} \in X_{1}$.

(1) If $M(\alpha)$ is a small 3-manifold which is a Seifert fibred space, then $\chi_{\rho}$ is a simple point of $X(M)$. Furthermore $\chi_{\rho}$ takes only real values on $\pi_{1}(M)$ and the eigenvalues of $\rho(\gamma)$ lie on either the real line or the unit circle.

(2) If $M(\alpha)$ has a finite fundamental group then $\chi_{\rho}$ is a simple point of $X(M)$. Furthermore the eigenvalues of $\rho(\delta)$ are roots of unity of order less than or equal to 10.

Proof. (1) By $[11, \S 1.5]$, the condition that $Z_{x}\left(\widetilde{f}_{\alpha}\right)>Z_{x}\left(\widetilde{f}_{\delta}\right)$ implies that there is some $\rho \in t^{-1}(\nu(x))$ with non-cyclic image in $\operatorname{PSL}(2, \mathbb{C})$ such that $\rho(\alpha) \in\{I,-I\}$. In fact $\rho$ can be chosen to have a nonabelian image, for the argument in [11] shows that $\rho$ can be chosen so that its image is also non-diagonalisable in $\operatorname{PSL}(2, \mathbb{C})$. Hence if its image is abelian, then it must conjugate into the group of uppertriangular parabolic matrices. But this cannot occur because the hypothesis that $M(\alpha)$ be small implies that $\operatorname{rank} H_{1}(M(\alpha) ; \mathbb{Z})=0$. Thus $\operatorname{rank} H_{1}(M ; \mathbb{Z})=1$ and therefore any parabolic representation has cyclic image in $P S L(2, \mathbb{C})$, contrary to our choices. 
Assume then, without loss of generality, that image $(\rho)$ is nonabelian. We can also assume that $\rho\left(\pi_{1}(M)\right)$ is nonabelian when projected to $P S L(2, \mathbb{C})$, for otherwise image $(\rho)$ would be the quaternion group of order 8 , and so again the proposition holds. Since $\rho(\alpha)= \pm I, \rho$ induces a representation $\pi_{1}(M(\alpha)) \rightarrow P S L(2, \mathbb{C})$ and our assumptions imply that the latter factors through a group $\Delta$ of the form $\langle a, b| a^{p}=b^{q}=$ $\left.(a b)^{r}=1\right\rangle$ for some $p, q, r \geq 2$. Choose $a_{1}, b_{1} \in \pi_{1}(M)$ which are sent to $a, b$ under the composition $\pi_{1}(M) \rightarrow \pi_{1}(M(\alpha)) \rightarrow \Delta$. Now fix $g \in G$. There is a word $w=w\left(a_{1}, b_{1}\right)$ such that $\rho(g)= \pm \rho(w)$ so that $\chi_{\rho}(g)= \pm \chi_{\rho}(w)$. It follows from [12, Proposition 1.4.1] that $\chi_{\rho}(w)=$ $P\left(\chi_{\rho}\left(a_{1}\right), \chi_{\rho}\left(b_{1}\right), \chi_{\rho}\left(a_{1} b_{1}\right)\right)$ where $P$ is a polynomial with integral coefficients. Now by construction, each of $\rho\left(a_{1}\right), \rho\left(b_{1}\right)$ and $\rho\left(a_{1} b_{1}\right)$ has finite order in $S L(2, \mathbb{C})$, and so have real traces. Thus $\chi_{\rho}(g)= \pm \chi_{\rho}(w) \in \mathbb{R}$. Finally, $\chi_{\rho}$ is a simple point of $X(M)$ by [5].

Fix $g \in G$. By part $(1), \operatorname{trace}(\rho(g))$ is real. Now $\rho(g)$ is conjugate to a matrix of the form

$$
\left(\begin{array}{cc}
u & * \\
0 & u^{-1}
\end{array}\right)
$$

Let $u=r e^{i \theta}$. Then $u+u^{-1}=r e^{i \theta}+\frac{1}{r} e^{-i \theta}=\left(r+\frac{1}{r}\right) \cos \theta+i\left(r-\frac{1}{r}\right) \sin \theta$ and so $\left(r-\frac{1}{r}\right) \sin \theta=0$. If $\sin \theta=0$, then $u$ is real. If $r-\frac{1}{r}=0$, then $r=1$ and $u$ lies on the unit circle.

(2) Again by $[11, \S 1.5]$, the condition that $Z_{x}\left(\tilde{f}_{\alpha}\right)>Z_{x}\left(\tilde{f}_{\delta}\right)$ implies that there is some $\rho \in t^{-1}(\nu(x))$ with non-cyclic image such that $\rho(\alpha) \in$ $\{I,-I\}$. Thus $\alpha$ has type $T, I, O, D$ or $Q$. By [5], $\chi_{\rho}$ is a simple point of $X(M)$. We can use $\rho$ to construct an irreducible representation $\bar{\rho}$ : $\pi_{1}(M(\alpha)) \rightarrow P S L(2, \mathbb{C})$. It follows from Lemma 3.1, and the discussion immediately preceding it, that $\rho\left(\pi_{1}(\partial M)\right)$ is a cyclic group of order no larger than 10. Thus part (2) holds.

q.e.d.

Let $A_{\mathrm{M}}(u, v)$ be the canonical $A$-polynomial of $M$ with respect to the basis $\{\mu, \lambda\}$.

Proposition 11.2. Suppose that $\alpha=(p, q) \in H_{1}(M ; \mathbb{Z})$ is a primitive class which is not a strict boundary class. Let $\left(u_{0}, v_{0}\right)$ be a solution of either

$$
\left\{\begin{array} { c } 
{ A _ { M } ( u , v ) = 0 } \\
{ u ^ { p } v ^ { q } = 1 }
\end{array} \quad \text { or } \quad \left\{\begin{array}{c}
A_{M}(u, v)=0 \\
u^{p} v^{q}=-1
\end{array}\right.\right.
$$

Assume that $M(\alpha)$ is a small 3-manifold.

(1) If one of $u_{0}$ or $v_{0}$ is not \pm 1 , then there is a representation $\rho \in$ $R(M)$, with nonabelian image, whose character lies in $C_{M}$ and for 
which

$$
\rho(\mu)=\left(\begin{array}{cc}
u_{0} & 0 \\
0 & u_{0}^{-1}
\end{array}\right) \text { and } \rho(\lambda)=\left(\begin{array}{cc}
v_{0} & 0 \\
0 & v_{0}^{-1}
\end{array}\right) .
$$

(2) If one of $u_{0}$ or $v_{0}$ is neither real nor on the unit circle, then $M(\alpha)$ is not a Seifert fibred space which is small.

(3) If one of $u_{0}$ or $v_{0}$ is not a root of unity of order less than or equal to 10 , then $\pi_{1}(M(\alpha))$ is not finite.

Proof. (1) We shall assume that $u_{0} \neq \pm 1$. The case $v_{0} \neq \pm 1$ is handled similarly.

As $\left(u_{0}, v_{0}\right)$ is a solution of $A_{\mathrm{M}}$, there is an algebraic component $X_{1}$ of $C_{\mathrm{M}}$ whose associated plane curve $D_{1}$ contains $\left(u_{0}, v_{0}\right)$. Recall from $\S 6$ that we have the following diagram of regular maps between affine algebraic sets:

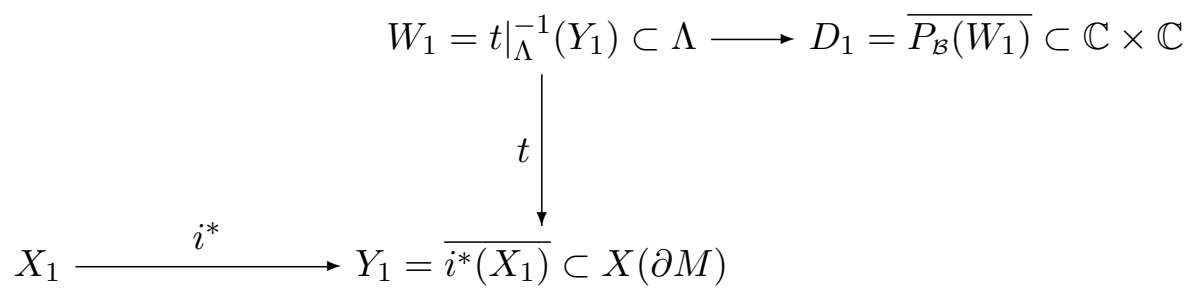

Hence we have an associated diagram of rational maps between smooth projective sets:

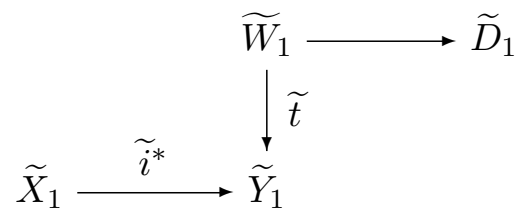

Each map in this latter diagram is surjective, so there is a point $w \in \widetilde{W}_{1}$ which is mapped to a point $d \in \nu^{-1}\left(u_{0}, v_{0}\right) \subset D_{1}^{\nu} \subset \widetilde{D}_{1}$. Fix any $x \in \widetilde{X}_{1}$ such that $\widetilde{i}^{*}(x)=\widetilde{t}(w) \in \widetilde{Y}$. Note that by construction $\widetilde{f}_{\mu}(x)=$ $\left(u_{0}-u_{0}^{-1}\right)^{2} \in \mathbb{C}^{*}$, while $\widetilde{f}_{\alpha}(x)=0$. Hence $Z_{x}\left(\widetilde{f}_{\mu}\right)=0<Z_{x}\left(\widetilde{f}_{\alpha}\right)$.

Assume first that $x \in \widetilde{X}_{1}-X_{1}^{\nu}$, i.e., $x$ is an ideal point. Since $\alpha$ is not a strict boundary class, [11, Prop. I.6.1] implies that there is an essential closed surface in $M$ which remains essential in $M(\alpha)$, contrary to our supposition that $M(\alpha)$ is a small 3-manifold. Thus $x \in X_{1}^{\nu}$. Set $\nu(x)=\chi_{\rho} \in X_{1}$ where $\rho \in R(M)$ and note that by 
the argument in the proof of [11, Proposition 1.5.5], we may assume that $\rho$ is not diagonalisable. Hence if the image of $\rho$ is abelian, then its non-central elements would have to consist of parabolic matrices. But this would imply that $u_{0}, v_{0} \in\{ \pm 1\}$. Hence $\rho$ is a nonabelian representation. Finally observe that since one of $u_{0}, v_{0}$ is different from \pm 1 , we can assume that $\rho \mid \pi_{1}(\partial M)$ is diagonal.

(2) We continue to use the notation of part (1). Since $M(\alpha)$ is a Seifert space which is small and $Z_{x}\left(\widetilde{f}_{\mu}\right)=0<Z_{x}\left(\widetilde{f}_{\alpha}\right)$, Proposition 11.1 implies that $\chi_{\rho}$ is a real valued function. But this contradicts the fact that either $\chi_{\rho}(\mu)=u_{0}+u_{0}^{-1} \notin \mathbb{R}$ or $\chi_{\rho}(\mu)=v_{0}+v_{0}^{-1} \notin \mathbb{R}$. Thus $M(\alpha)$ cannot be a Seifert space which is small.

Part (3) follows from a similar argument.

q.e.d.

In our next two results we see how the existence of finite filling slopes of a given type constrains the $A$-polynomial.

Proposition 11.3. Suppose that $\alpha=p \mu+q \lambda \in L$ is an I(5)-type finite filling class which is not a strict boundary class. Suppose further that $\|\alpha\|_{M}>s_{M}$. The following statements hold:

(1) There are integers $a, b, c, j$ where $j \geq 1$ and $\theta \in\{ \pm 1\}$ for which $A_{M}\left(z^{q}, z^{-p}\right)= \pm z^{a}(z-1)^{b}(z+1)^{c}\left(z^{4}+\theta z^{3}+z^{2}+\theta z+1\right)^{j}$.

(2) Let $\epsilon$ be the nonzero element of $H^{1}\left(M ; \mathbb{Z}_{2}\right)=\mathbb{Z}_{2}$ and choose $\theta \in$ $\{ \pm 1\}$ so that $A_{M}\left(z^{q}, z^{-p}\right)= \pm z^{a}(z-1)^{b}(z+1)^{c}\left(z^{4}+\theta z^{3}+z^{2}+\right.$ $\theta z+1)^{j}$. Then:

(i) If $q$ is odd, $A_{M}\left(z^{q},-z^{-p}\right)= \pm z^{a}(z-\epsilon(\mu))^{b}(z+\epsilon(\mu))^{c}\left(z^{4}+\right.$ $\left.\epsilon(\mu) \theta z^{3}+z^{2}+\epsilon(\mu) \theta z+1\right)^{j}$.

(ii) If $p$ is odd, $A_{M}\left(-z^{q}, z^{-p}\right)= \pm z^{a}(z-\epsilon(\lambda))^{b}(z+\epsilon(\lambda))^{c}\left(z^{4}+\right.$ $\left.\epsilon(\lambda) \theta z^{3}+z^{2}+\epsilon(\lambda) \theta z+1\right)^{j}$.

Remark 11.4. It can be shown that the power $j$ which occurs in Proposition 11.3, and in the proposition which follows, is 1. As we won't be needing this in the proof of the finite filling conjecture, we will only prove the simpler statements.

Proof. According to Lemma 2.1, $H_{1}\left(M ; \mathbb{Z}_{2}\right)=\mathbb{Z}_{2}$ and further $i_{*}(\alpha)$ is a generator of this group. In what follows, $\epsilon$ will denote the nonzero element of $H_{1}\left(M ; \mathbb{Z}_{2}\right)$. Clearly we have $\epsilon(\alpha)=-1$.

The hypothesis $\|\alpha\|_{\mathrm{M}}>s_{\mathrm{M}}$ implies that there is a point $x \in \widetilde{C}_{\mathrm{M}}$ such that $Z_{x}\left(\widetilde{f}_{\alpha}\right)>Z_{x}\left(\widetilde{f}_{\mu}\right)$. Since $\pi_{1}(M(\alpha))$ is finite, [11, Proposition 1.6.1] implies that $x \in C_{\mathrm{M}}^{\nu}$. If we set $\nu(x)=\chi_{\rho_{0}} \in C_{\mathrm{M}}$, then from $[11, \S 1.5]$ it 
follows that we can assume that $\rho_{0}$ is nonabelian and that $\rho_{0}(\alpha)= \pm I$. By possibly replacing $\rho_{0}$ by $\epsilon \cdot \rho_{0}$ we may arrange for $\rho_{0}(\alpha)=I$. Since $\alpha$ has type $I(5)$ and $\rho_{0}$ factors through $\pi_{1}(M(\alpha)), \rho_{0}(\pi) \subset S L(2, \mathbb{C})$ is isomorphic to the binary icosahedral group (see $\S 3$ ) and $\rho_{0}\left(\pi_{1}(\partial M)\right)$ has image $\mathbb{Z}_{5}$ in $P S L(2, \mathbb{C})$. After conjugating $\rho_{0}$ by an element of $S L(2, \mathbb{C})$ we may arrange for its restriction to $\pi_{1}(\partial M)$ to be diagonal, say

$$
\rho_{0}(\mu)=\left(\begin{array}{cc}
u_{0} & 0 \\
0 & u_{0}^{-1}
\end{array}\right), \rho_{0}(\lambda)=\left(\begin{array}{cc}
v_{0} & 0 \\
0 & v_{0}^{-1}
\end{array}\right) .
$$

Choose integers $s, t$ so that $q s-p t=1$ and set $z_{0}=u_{0}^{s} v_{0}^{t}$. Then $z_{0}^{q}=u_{0}$ and $z_{0}^{-p}=v_{0}$ so $A_{\mathrm{M}}\left(z_{0}^{q}, z_{0}^{-p}\right)=0$. Further it is clear that $\pm \rho_{0}\left(\pi_{1}(\partial M)\right) \cong \mathbb{Z}_{5} \subset P S L(2, \mathbb{C})$ is generated by $\pm \rho_{0}(s \mu+t \lambda)=$ $\left(\begin{array}{cc}z_{0} & 0 \\ 0 & z_{0}^{-1}\end{array}\right)$. Thus $z_{0}$ is a primitive $n^{\text {th }}$ root of unity where $n=$ $\left|\rho_{0}\left(\pi_{1}(\partial M)\right)\right| \in\{5,10\}$. Since $A_{\mathrm{M}}$ is an integral polynomial it follows that $A_{\mathrm{M}}\left(z^{q}, z^{-p}\right)$ is divisible by the irreducible polynomial of $z_{0}$, which is $z^{4}+z^{3}+z^{2}+z+1$ if $n=5$ or $z^{4}-z^{3}+z^{2}-z+1$ if $n=10$. To complete the proof of part (1) of the proposition, we must show that any nonzero root $z_{1}$ of $A_{\mathrm{M}}\left(z^{q}, z^{-p}\right)$, different from \pm 1 , is also a primitive $n^{\text {th }}$ root of unity.

Let $z_{1}$ be such a root and note that $\left(z_{1}^{q}\right)^{s}\left(z_{1}^{-p}\right)^{t}=z_{1}^{q s-p t}=z_{1} \neq \pm 1$. Thus if $\left(u_{1}, v_{1}\right)=\left(z_{1}^{q}, z_{1}^{-p}\right)$, then one of $u_{1}, v_{1}$ is different from \pm 1 . By Proposition 11.2 there is a character $\chi_{\rho_{1}} \in C_{\mathrm{M}}$ of a nonabelian representation $\rho_{1} \in R(M)$ such that

$$
\rho_{1}(\mu)=\left(\begin{array}{cc}
u_{1} & 0 \\
0 & u_{1}^{-1}
\end{array}\right), \rho_{1}(\lambda)=\left(\begin{array}{cc}
v_{1} & 0 \\
0 & v_{1}^{-1}
\end{array}\right) .
$$

Therefore $\rho_{1}(\alpha)=I$. Now it is shown in $[2, \S 5]$ that up to conjugacy there are precisely four nonabelian representations $\rho: \pi \rightarrow S L(2, \mathbb{C})$ such that $\rho(\alpha)= \pm I$. In fact these four representations are given by $\rho_{0}, \epsilon \cdot \rho_{0}, \phi \circ \rho_{0}$ and $\epsilon \cdot \phi \circ \rho_{0}$ where $\phi$ is a nontrivial outer automorphism of the image of $\rho_{0}$ (the binary icosahdral group). Since $\epsilon(\alpha)=-1$, it follows that up to conjugation there are exactly two nonabelian representations $\rho \in R(M)$ satisfying $\rho(\alpha)=I$ and they are $\rho_{0}$ and $\phi \circ \rho_{0}$. In particular $\rho_{1}$ is conjugate to one of these two representations. In either case, $u_{1}$ is a primitive $m^{\text {th }}$ root of unity where $m=\left|\rho_{1}\left(\pi_{1}(\partial M)\right)\right|=\left|\rho_{0}\left(\pi_{1}(\partial M)\right)\right|=n$. This completes the proof of part (1) of the proposition.

To prove part (2) choose $\theta \in\{ \pm 1\}$ and integers $a, b, c, j$ so that $A_{\mathrm{M}}\left(z^{q}, z^{-p}\right)= \pm z^{a}(z-1)^{b}(z+1)^{c}\left(z^{4}+\theta z^{3}+z^{2}+\theta z+1\right)^{j}$. Since 
$\epsilon\left(D_{\mathrm{M}}\right)=D_{\mathrm{M}}$, we see that $A_{\mathrm{M}}(u, v)= \pm A_{\mathrm{M}}(\epsilon(\mu) u, \epsilon(\lambda) v)$. Now $\epsilon(\alpha)=$ -1 and so if $q$ is odd then $\epsilon(\lambda)=\epsilon(\lambda)^{q}=-\epsilon(\mu)^{p}$. Therefore $A_{\mathrm{M}}(u, v)=$ $\pm A_{\mathrm{M}}(\epsilon(\mu) u, \epsilon(\lambda) v)= \pm A_{\mathrm{M}}\left(\epsilon(\mu) u,-\epsilon(\mu)^{p} v\right)$. Thus $A_{\mathrm{M}}\left(z^{q}, z^{-p}\right)=$ $\pm A_{\mathrm{M}}\left(\epsilon(\mu) z^{q},-(\epsilon(\mu) z)^{-p}\right)= \pm A_{\mathrm{M}}\left((\epsilon(\mu) z)^{q},-(\epsilon(\mu) z)^{-p}\right)$. In particular the roots of $A_{\mathrm{M}}\left(z^{q}, z^{-p}\right)$, counted with multiplicity, correspond bijectively to those of $A_{\mathrm{M}}\left(z^{q},-z^{-p}\right)$ under the function which sends a zero $z_{1}$ of $A_{\mathrm{M}}\left(z^{q}, z^{-p}\right)$ to the root $\epsilon(\mu) z_{1}$ of $A_{\mathrm{M}}\left(z^{q},-z^{-p}\right)$. Hence $A_{\mathrm{M}}\left(z^{q},-z^{-p}\right)$ $= \pm z^{a}(z-\epsilon(\mu))^{b}(z+\epsilon(\mu))^{c}\left(z^{4}+\epsilon(\mu) \theta z^{3}+z^{2}+\epsilon(\mu) \theta z+1\right)^{j}$. A similar argument works if $p$ is odd. This completes the proof of the proposition.

q.e.d.

Similarly one can show:

Proposition 11.5. Suppose that $\alpha=p \mu+q \lambda \in L$ is a T(3)-type finite filling class which is not a strict boundary class. Suppose further that $\|\alpha\|_{M}>s_{M}$. The following statements hold:

(1) There are integers $a, b, c, j$ where $j \geq 1$ and $\theta \in\{ \pm 1\}$ for which $A_{M}\left(z^{q}, z^{-p}\right)= \pm z^{a}(z-1)^{b}(z+1)^{c}\left(z^{2}+\theta z+1\right)^{j}$.

(2) Let $\epsilon$ be the nonzero element of $H^{1}\left(M ; \mathbb{Z}_{2}\right)=\mathbb{Z}_{2}$ and choose $\theta \in$ $\{ \pm 1\}$ so that $A_{M}\left(z^{q}, z^{-p}\right)= \pm z^{a}(z-1)^{b}(z+1)^{c}\left(z^{2}+\theta z+1\right)^{j}$. Then:

(i) If $q$ is odd, $A_{M}\left(z^{q},-z^{-p}\right)= \pm z^{a}(z-\epsilon(\mu))^{b}(z+\epsilon(\mu))^{c}\left(z^{2}+\right.$ $\epsilon(\mu) \theta z+1)^{j}$.

(ii) If $p$ is odd, $A_{M}\left(-z^{q}, z^{-p}\right)= \pm z^{a}(z-\epsilon(\lambda))^{b}(z+\epsilon(\lambda))^{c}\left(z^{2}+\right.$ $\epsilon(\lambda) \theta z+1)^{j}$.

Example 11.6. Let $M$ be the exterior of the $(-2,3,7)$-pretzel knot. It is known [1] that $M$ is hyperbolic and that if $\mu, \lambda$ are the standard meridian-longitude coordinates for knots in the 3 -sphere then the $17 \mu+\lambda$-filling of $M$ has fundamental group $I_{120} \times \mathbb{Z} / 17$. It is also known that 18 and 19-fillings yield lens spaces. The canonical $A$-polynomial of $M$, with respect to $\mu, \lambda$, has been calculated in [8] to be

$$
\begin{aligned}
A_{\mathrm{M}}(u, v)= & -1+\left(u^{16}-2 u^{18}+u^{20}\right) v+\left(2 u^{36}+u^{38}\right) v^{2} \\
& +\left(-u^{72}-2 u^{74}\right) v^{4}+\left(-u^{90}+2 u^{92}-u^{94}\right) v^{5}+u^{110} v^{6} .
\end{aligned}
$$

From the polynomial and its Newton polygon we see that the minimal norm $s_{\mathrm{M}}=12$ and further that the norm of the slope 17 is $20=s_{\mathrm{M}}+8$. Specializing the variables to $v=z^{-17}$ and $v=-z^{-17}$ yields

$$
A_{\mathrm{M}}\left(z, z^{-17}\right)=-\left(z^{4}-z^{3}+z^{2}-z+1\right)(z-1)^{3}(z+1)^{3} z^{-1}
$$


in the former case and

$$
A_{\mathrm{M}}\left(z,-z^{-17}\right)=-\left(z^{4}+z^{3}+z^{2}+z+1\right)(z-1)^{3}(z+1)^{3} z^{-1}
$$

in the latter. Setting $u=z$ and $v= \pm z^{-18}$ or $\pm z^{-19}$ yields Laurent polynomials whose roots are \pm 1 .

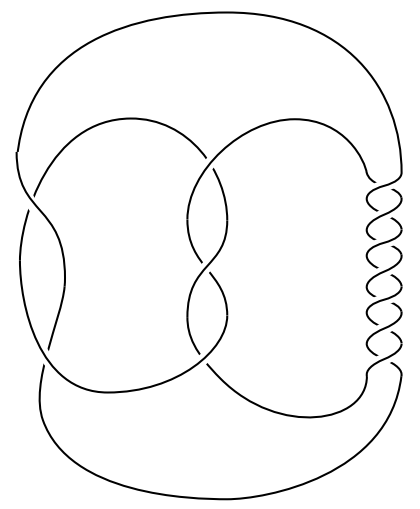

Figure 4: The $(-2,3,7)$-pretzel knot.

Example 11.7. In our second example we take $M$ to be the exterior of the knot $K$ in the lens space $L(5,1)$ obtained by Dehn filling one of the boundary tori of the Whitehead link exterior with the slope 5 (with respect to the standard meridian-longitude coordinates). It is commonly referred to as the figure-8 sister knot. This manifold is also hyperbolic and admits five finite Dehn fillings [35]: The two fillings parameterized by $3 \mu+\lambda$ and $3 \mu+2 \lambda$ are of $T$-type, while those parameterized by $2 \mu+\lambda, \mu+\lambda$ and $\mu$-fillings are lens spaces. A computer-aided calculation shows

$$
A_{\mathrm{M}}(u, v)=1+\left(u^{2}-u^{4}\right) v-2 u^{4} v^{2}+\left(-u^{4}+u^{6}\right) v^{3}+u^{8} v^{4}
$$

and so $s_{\mathrm{M}}=8$ while the norms of both $3 \mu+\lambda$ and $3 \mu+2 \lambda$ are $12=s_{\mathrm{M}}+4$. Corresponding to the $T$-type filling associated to $3 \mu+\lambda$ we have

$$
A_{\mathrm{M}}\left(z, z^{-3}\right)=-\left(z^{2}-z+1\right)(z-1)^{2}(z+1)^{2} z^{-5}
$$

while

$$
A_{\mathrm{M}}\left(z,-z^{-3}\right)=\left(z^{2}+z+1\right)(z-1)^{2}(z+1)^{2} z^{-5} .
$$

Corresponding to the $T$-type filling slope $3 \mu+2 \lambda$ we have

$$
A_{\mathrm{M}}\left(z^{2}, z^{-3}\right)=A_{\mathrm{M}}\left(-z^{2}, z^{-3}\right)=-\left(z^{2}-z+1\right)(z-1)^{2}(z+1)^{2} z^{-1} .
$$




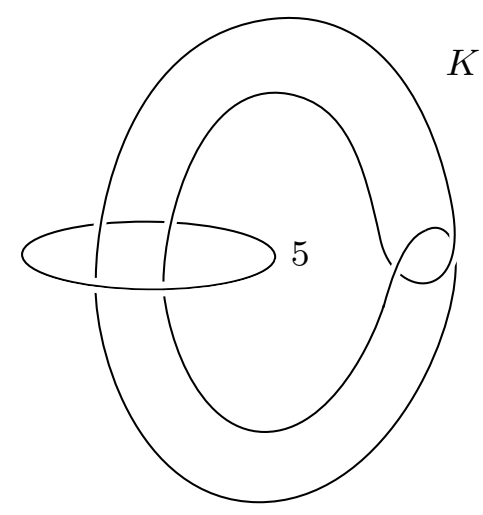

Figure 5: The figure-8 sister knot.

\section{Outline of the proof of the conjecture}

From now till the end of the paper $\mu \in L$ will denote a class satisfying $\|\mu\|_{M}=s_{M}$. We shall also assume that either

- $\mu$ is not a vertex of $B_{\mathrm{M}}$, or

- each class in $\partial B_{\mathrm{M}} \cap L$ is a vertex of $B_{\mathrm{M}}$.

Let $\lambda \in L$ be a class such that $\{\mu, \lambda\}$ is a basis for $L$. We parameterize the slopes on $\partial M$ by the set of primitive classes $p \mu+q \lambda \in L$ where either $q>0$ or $q=0$ and $p=1$.

A class in $L$ will be called integral if its $\lambda$ coordinate is \pm 1 .

Define $\mathcal{F}_{0}$ to be the set of finite or cyclic filling classes $\alpha=p \mu+q \lambda$ which are not strict boundary classes.

Theorem 12.1. Let \#, $\Delta$, and $n_{0}$ denote respectively the number, the maximal mutual distance, and the maximal value of the $\lambda$ coordinates of all classes in $\mathcal{F}_{0}$. Then $\# \leq 5, \Delta \leq 3, n_{0} \leq 2$. Further $\Delta=3$ can be realized by at most one pair of classes.

Assuming Theorem 12.1 holds, we may complete the proof of the finite filling conjecture as follows.

Proof of the finite filling conjecture. Denote by $\mathcal{F}$ the set of all finite or cyclic filling classes and by $\mathcal{S}$ the set of all strict boundary classes. If $\mathcal{F} \cap \mathcal{S}=\emptyset$, then the conjecture follows from Theorem 12.1. Suppose next that $\mathcal{F} \cap \mathcal{S}=\{\alpha\}$. By Proposition 2.2, any finite or cyclic class has distance at most 1 from $\alpha$. Hence if $\alpha=\mu$, then all other finite or 
cyclic classes are integral and so by Theorem 12.1, their number is at most four and their maximal mutual distance is at most 3 , realized at most once. This implies that the conjecture holds. Similarly if $\alpha \neq \mu$, the truth of the conjecture is easy to verify. Finally, if $\mathcal{F} \cap \mathcal{S} \supset\left\{\alpha_{1}, \alpha_{2}\right\}$, then it follows from Proposition 2.2 that $\# \leq 4$ and $\Delta \leq 2$. q.e.d.

The rest of the paper is devoted to the proof of Theorem 12.1. One easily sees that it is a consequence of the following five propositions (the inequality $n_{0} \leq 2$ has been proved in [2, Theorem 7.2]). Recall that $4 \leq s_{M} \equiv 0(\bmod 2)$ (Lemma 9.2$)$.

Proposition 12.2. If $s_{M} \leq 6$ then $\# \leq 5, \Delta \leq 3$ and there are at most two classes in $\mathcal{F}_{0}$ whose mutual distance is 3 .

Proposition 12.3. When $s_{M} \geq 8$, there is at most one class in $\mathcal{F}_{0}$ whose $\lambda$-coordinate is equals 2 .

Proposition 12.4. When $s_{M} \geq 8$, there are at most four integral classes in $\mathcal{F}_{0}$ and their maximal distance is at most three.

Proposition 12.5. When $s_{M} \geq 8, \Delta \leq 3$.

Proposition 12.6. When $s_{M} \geq 8$, there is at most one pair of classes $\alpha$ and $\beta$ in $\mathcal{F}_{0}$ for which $\Delta(\alpha, \beta)=3$.

These propositions will be proved in the next five sections. Throughout, $i: \partial M \rightarrow M$ will be the inclusion and $i_{*}: H_{1}\left(\partial M ; \mathbb{Z}_{2}\right) \rightarrow$ $H_{1}\left(M ; \mathbb{Z}_{2}\right)$ the induced homomorphism.

\section{Proof of Proposition 12.2}

We shall divide the proof of Proposition 12.2 into two main cases: (I) $s_{\mathrm{M}}=4$ and (II) $s_{\mathrm{M}}=6$.

Case I. $s_{M}=4$.

By Lemma $9.2, H_{1}\left(M ; \mathbb{Z}_{2}\right)=\mathbb{Z}_{2}$ and by Proposition $9.3, \mathcal{F}_{0} \subset 3 B_{\mathrm{M}}$. We shall divide Case I into two subcases depending on whether or not $\mu$ is a vertex of $B_{\mathrm{M}}$.

Subcase I.1. $\mu$ is not a vertex of $B_{\mathrm{M}}$.

It then follows from [2, Lemma 6.5] that $C_{M}$ is an irreducible component of $X(M)$, and $B_{M}$ is a parallelogram with vertices $\pm(a, 2 /(k+2))$ and $\pm(a+2,2 /(k+2))$, for some integer $k \geq 0$ satisfying $a(k+2) / 2 \in \mathbb{Z}$. In fact using the Newton polygon we can find a much stronger constraint on $k$. 
Lemma 13.1. $k \equiv 2(\bmod 4)$.

Proof. According to Proposition $9.6(2)$, we must have $i_{*}(\mu) \neq 0$. We claim first that $k$ must be even. We may assume that $k>0$ and so from the shape of $B_{\mathrm{M}}$, there exist two consecutive integral classes in $L$, say $\alpha_{1}=(m, 1)$ and $\alpha_{2}=(m+1,1)$, such that neither $\alpha_{1}$ nor $\alpha_{2}$ is a boundary class corresponding to a vertex of $B_{\mathrm{M}}$ and that $\left\|\alpha_{1}\right\|_{\mathrm{M}}=$ $\left\|\alpha_{2}\right\|_{\mathrm{M}}=2(k+2)$. Since $i_{*}(\mu) \neq 0$, one of $\alpha_{1}$ and $\alpha_{2}$, say $\alpha_{1}$, must lie in the kernel of $i_{*}$. Hence applying Proposition 9.6, we see that $\left\|\alpha_{1}\right\|_{\mathrm{M}}$ is divisible by 4 , and so $k$ is even.

Now we replace $\lambda$ by the class corresponding to $\left(\frac{(k+2)}{2} a+\frac{(k+2)}{2}, 1\right)$. Then $B_{\mathrm{M}}$ becomes the parallelogram whose vertices are $\pm\left(-1, \frac{2}{(k+2)}\right)$ and $\pm\left(1, \frac{2}{(k+2)}\right)$. It follows from Proposition 10.1 that the Newton polygon is a parallelogram whose vertices are $(0,1),(k+2,1),\left(\frac{(k+2)}{2}, 0\right)$ and $\left(\frac{(k+2)}{2}, 2\right)$ (the case $k=4$ is depicted in Figure 6 ). In particular $a_{\frac{(k+2)}{2}, 0} \neq 0$. Since $i_{*}(\mu) \neq 0$, Proposition 10.3 implies that $\frac{(k+2)}{2}$ is even. Thus $k \equiv 2(\bmod 4)$.

q.e.d.

When $k=2$, one can easily check from the shape of $B_{M}$ together with Proposition 9.3 , that $\# \leq 4, \Delta \leq 2$ (recall $\mathcal{F}_{0} \subset 3 B_{\mathrm{M}}$ ). Similarly when $k \geq 6$ the only possible class in $\mathcal{F}_{0}$ is $\mu$. Thus Proposition 12.2 holds in Subcase I.1.
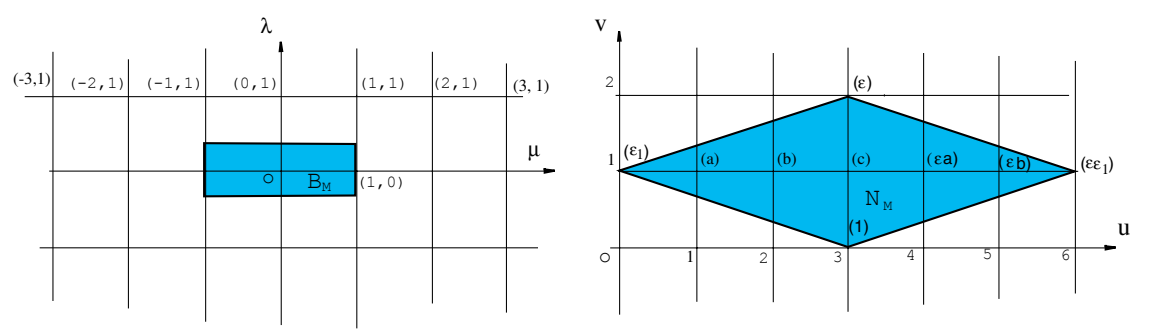

Figure 6: The canonical norm polygon and Newton polygon when $k=4$ in Subcase I.1.

Subcase I.2. $\mu$ is a vertex of $B_{\mathrm{M}}$.

By the convention set at the beginning of $\S 12$, all points of $\partial B_{M} \cap L$ are vertices of $B_{M}$. Since $s_{\mathrm{M}}=4, C_{\mathrm{M}}$ is either irreducible or contains 

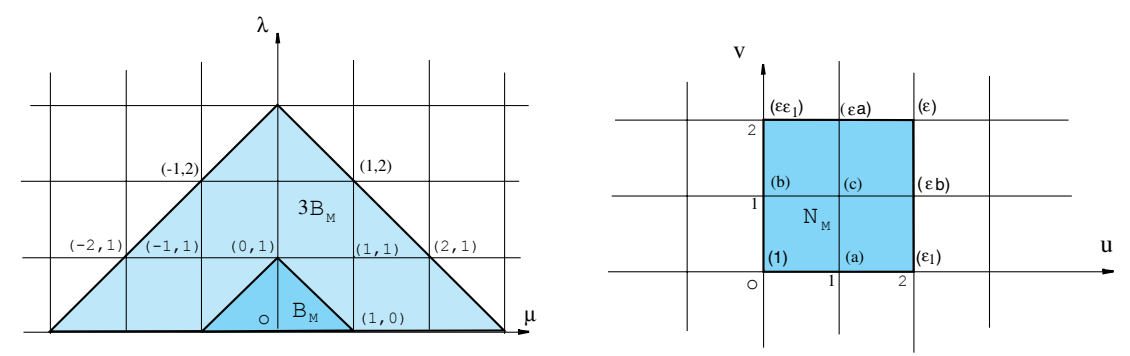

Figure 7: The canonical norm polygon and Newton polygon when $k=0$ in Subcase I.2.a.

exactly two components, i.e., $C_{\mathrm{M}}=X_{1}$ or $C_{\mathrm{M}}=X_{1} \cup X_{2}$. According to [2, Lemma $6.6(2)$ ] (which remains true for $B_{M}$ since $B_{\mathrm{M}}=B_{1}$ by Proposition 9.1), there are three types possible for the shape of $B_{\mathrm{M}}$.

Subcase (I.2.a). $\quad B_{M}$ is a parallelogram with vertices $\pm(1,0)$ and $\pm\left(\frac{2 m}{k+2}, \frac{2}{k+2}\right)$ for some integers $m$ and $k \geq 0$.

We may assume that $m=0$. When $k \geq 1$, the fact that $\mathcal{F}_{0} \subset 3 B_{M}$ is sufficient to obtain the estimates $\Delta \leq 2$ and $\# \leq 2$. Suppose then that $k=0$. In this case $B_{M}$ is a parallelogram with vertices $\pm(1,0)$ and $\pm(0,1)$ (Figure 7$)$. Since $\mathcal{F}_{0} \subset 3 B_{M}$ consists of non- $\partial$-slopes, $\mathcal{F}_{0} \subset\{(-1,2),(1,2),(-2,1),(-1,1),(1,1),(2,1)\}$. Now by Proposition 10.1, the canonical Newton polygon $N_{\mathrm{M}}$ is as shown in Figure 7 and by Proposition 10.3 (1)-(3), the canonical $A$-polynomial of $M$ with respect to the basis $\mathcal{B}=\{\mu, \lambda\}$ is of the form

$$
A_{M}(u, v)=1+a u+\epsilon_{1} u^{2}+\left(b+c u+\epsilon b u^{2}\right) v+\left(\epsilon \epsilon_{1}+\epsilon a u+\epsilon u^{2}\right) v^{2},
$$

where $a, b \in \mathbb{Z}$ and $\epsilon, \epsilon_{1} \in\{ \pm 1\}$. Since $\|\mu\|_{\mathrm{M}}=\|\lambda\|_{\mathrm{M}}=4$, the zero sets in $\widetilde{C}_{\mathrm{M}}$ of both $f_{\mu}$ and $f_{\lambda}$ consists of the four discrete faithful characters of $\pi_{1}(M)$. In particular, neither $f_{\mu}$ nor $f_{\lambda}$ has a zero at an ideal point of $\widetilde{C}_{\mathrm{M}}$. Therefore by Proposition $10.3(4),+1$ and -1 are not zeros of any edge polynomial of $A_{\mathrm{M}}(u, v)$. At least one of $\mu$ and $\lambda$ is not contained in the kernel of $i_{*}$ and without loss of generality we take $i_{*}(\mu) \neq 0$. Then by Proposition 10.3 (4) and (5), we have $a=0$ and since \pm 1 are not roots of the edge polynomials of $N_{\mathrm{M}}$, we have $\epsilon_{1}=1$. Hence $A_{M}(u, v)=1+u^{2}+\left(b+c u+\epsilon b u^{2}\right) v+\left(\epsilon+\epsilon u^{2}\right) v^{2}$. We now use $A_{M}(u, v)$ to show:

Lemma 13.2. When $k=0, \alpha=(-2,1)$ is not a finite filling class. 
Proof. Suppose otherwise. From Figure 7, we see that $\|\alpha\|_{\mathrm{M}}=$ $s_{\mathrm{M}}+8$ and $\alpha$ must be an I(5)-type class by Proposition 9.3 (recall $\left.H_{1}\left(M ; \mathbb{Z}_{2}\right)=\mathbb{Z}_{2}\right)$. Also by Proposition 11.3 there is an integer $d \geq 1$ for which $A_{M}\left(u, u^{2}\right)=1+(b+1) u^{2}+c u^{3}+\epsilon(b+1) u^{4}+\epsilon u^{6}$ is divisible by either $\left(u^{4}+u^{3}+u^{2}+u+1\right)^{j}$ or $\left(u^{4}-u^{3}+u^{2}-u+1\right)^{j}$ and the only roots of the quotient are \pm 1 . Obviously $j=1$.

Fix $\theta \in\{ \pm 1\}$ and suppose that $A_{M}\left(u, u^{2}\right)$ is divisible by $u^{4}+\theta u^{3}+$ $u^{2}+\theta u+1$. Long division yields the quotient $\epsilon u^{2}-\epsilon \theta u+\epsilon(b+1)$. But since the coefficient of $u$ is odd, $\epsilon u^{2}-\epsilon \theta u+\epsilon(b+1)$ has roots other than \pm 1 . This contradiction completes the proof of the lemma. q.e.d.

Similarly one can show that none of the classes $(2,1),(1,2)$ and $(-1,2)$ can be in $\mathcal{F}_{0}$. Thus when $k=0$, we have $\Delta \leq 2$ and $\# \leq 2$.

Subcase (I.2.b). $\quad B_{M}$ is a parallelogram with vertices $\pm(1,0)$ and $\pm\left(\frac{2(2 j+1)}{k+2}, \frac{4}{k+2}\right)$, for some integers $j$ and $k \geq 2$.

In this case, one can easily verify that $\mathcal{F}_{0} \cap 3 B_{M}$ contains at most four classes, the distance between any two of them is at most three, and the distance three is realized by at most one pair of these classes.

Subcase (I.2.c). $B_{M}$ is a polygon with vertices $\pm(1,0), \pm\left(\frac{2 m}{k+2}, \frac{2}{k+2}\right)$, $\pm\left(\frac{2(m+j)}{k+2}, \frac{2}{k+2}\right)$, for some integers $m, j, k$ with $j \geq 1$ and $k \geq j-1$.

We may assume that $m=0$. By the dual relationship between $B_{\mathrm{M}}$ and $N_{\mathrm{M}}$ (Proposition 10.1), the latter has vertices $(0,0),(0,1),(j, 2),(k+$ $2,2),(k+2,1)$ and $(k+2-j, 0)$ (see Figure 8$)$. In particular the coefficient of $v$ in $A_{\mathrm{M}}$ is nonzero. Thus by Proposition $10.3(5)$ we see that $i_{*}(\lambda)=$ 0 . Hence $i_{*}(\mu) \neq 0$ and so applying the same result we see that no odd powers of $u$ occur in $A_{\mathrm{M}}(u, v)$. Consideration of the coefficients corresponding to the vertices $(j, 2)$ and $(k+2,2)$, we see that both $j$ and $k$ are even. In particular this implies $j, k \geq 2$. When $k \geq 4$, one readily verifies that Proposition 12.2 is a consequence of Proposition 9.3, so the only case we need consider is when $k=j=2$.

Suppose then that $k=j=2$. The only classes in $3 B_{M}$ which can lie in $\mathcal{F}_{0}$ are $(-1,1),(1,1)$ and $(3,1)$ (Figure 8$)$. We need only show that $(-1,1)$ is not a finite filling class. From the previous paragraph we see that the canonical $A$-polynomial is of the form

$$
A_{M}(u, v)=1+\epsilon_{1} u^{2}+\left(\epsilon_{2}+c u^{2}+\epsilon \epsilon_{2} u^{4}\right) v+\left(\epsilon \epsilon_{1} u^{2}+\epsilon u^{4}\right) v^{2}
$$

where $c \in \mathbb{Z}$ and $\epsilon, \epsilon_{1}, \epsilon_{2} \in\{ \pm 1\}$. Since $\|\mu\|_{\mathrm{M}}=4, \widetilde{f}_{\mu}$ has no zeros at ideal points of $\widetilde{C}_{\mathrm{M}}$. Hence by Proposition 10.3 (4) we have $\epsilon_{1}=1$. So $A_{M}(u, v)=1+u^{2}+\left(\epsilon_{2}+c u^{2}+\epsilon \epsilon_{2} u^{4}\right) v+\left(\epsilon u^{2}+\epsilon u^{4}\right) v^{2}$. 

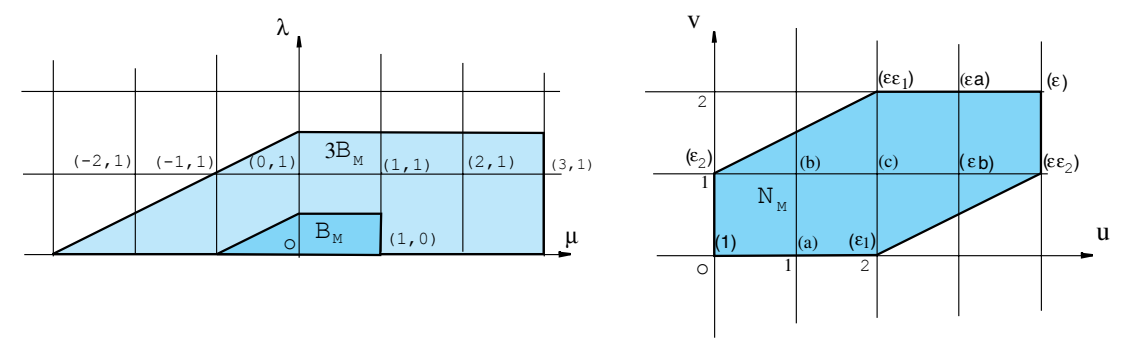

Figure 8: The canonical norm polygon and Newton polygon when $k=$ $j=2$ in Subcase I.2.c.

Lemma 13.3. When $k=j=2, \alpha=(-1,1)$ is not a finite filling class.

Proof. Suppose otherwise. From the shape of $B_{\mathrm{M}}$, we see that $\|\alpha\|_{\mathrm{M}}=s_{\mathrm{M}}+8$ and therefore $\alpha$ must be an $I(5)$-type class (recall $\left.H_{1}\left(M ; \mathbb{Z}_{2}\right)=\mathbb{Z}_{2}\right)$. Then by Proposition 11.3, $A_{M}(u, u)=1+\epsilon_{2} u+$ $u^{2}+c u^{3}+\epsilon u^{4}+\epsilon \epsilon_{2} u^{5}+\epsilon u^{6}$ is divisible by either $u^{4}+u^{3}+u^{2}+u+1$ or $u^{4}-u^{3}+u^{2}-u+1$, and the roots of the quotient are \pm 1 . But for $\theta \in\{ \pm 1\}$, the quotient of $A_{M}(u, u)$ by $u^{4}+\theta u^{3}+u^{2}+\theta u+1$ is $q(u)=\epsilon u^{2}+\epsilon\left(\epsilon_{2}-\theta\right) u+\epsilon\left(1-\theta \epsilon_{2}\right)$. Since the leading term of $q(u)$ is not congruent $(\bmod 2)$ to its constant term, it has a root other than \pm 1 . Thus $\alpha=(-1,1)$ cannot be a finite filling class. $\quad$ q.e.d.

Case II. $s_{M}=6$.

In this case we also have $H_{1}\left(M ; \mathbb{Z}_{2}\right)=\mathbb{Z}_{2}$ (Lemma 9.2). Therefore by Proposition $9.3, \mathcal{F}_{0} \subset \frac{7}{3} s_{M}$. We consider two subcases.

Subcase II.1. $\mu$ is not a vertex of $B_{\mathrm{M}}$.

By [2, Lemma $6.5(2)]$, there are three subcases to consider.

Subcase (II.1.a). $\quad B_{M}$ is a parallelogram with vertices $\pm(3(2 m+$ $1) /|k|, 6 /|k|)$ and $\pm(3(2 m+1+k) / 2|k|, 3 /|k|)$, for some integer $m$ and odd integer $k$, with $|k| \geq 5$.

After an appropriate change in $\lambda$, we may assume that $m=0$. By symmetry, we may also suppose that $k \leq-5$.

According to Proposition 10.1, $N_{\mathrm{M}}$ has vertices $(0,1),(1,3),\left(\frac{1-k}{2}, 2\right)$ and $\left(\frac{-(1+k)}{2}, 0\right)$ (the case $k=-5$ is pictured in Figure 9$)$. Since $(0,1)$ is a vertex, Proposition $10.3(5)$ implies that $i_{*}(\lambda)=0$, so $i_{*}(\mu) \neq 0$. But then the same result implies that no odd power of $u$ occurs in $A_{\mathrm{M}}$, 
contrary to the fact that $(1,3)$ is a vertex of $N_{\mathrm{M}}$. Thus this case cannot arise.
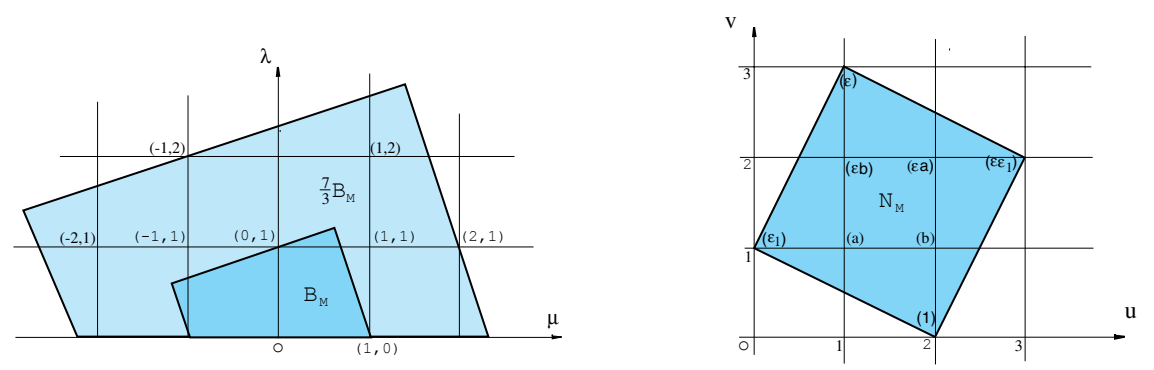

Figure 9: The canonical norm polygon and Newton polygon when $k=$ -5 in Subcase II.1.a.

Subcase (II.1.b). $B_{M}$ is a parallelogram with vertices $\pm(3 m /|k|, 3 /|k|)$ and $\pm(3(m+k) / 2|k|, 3 / 2|k|)$, for some integers $m$ and $k$, with $|k| \geq 3$.

We may assume that $m=0$ and $k \geq 3$. If $k \geq 7$, the primitive classes in $\frac{7}{3} B_{\mathrm{M}}$ are a subset of $\{ \pm \mu, \pm \lambda\}$, and so we need only consider $k=3,4,5$ and 6 . The cases $k=3,5,6$ can be handled in a straightforward manner using Proposition 9.3 alone, so we shall assume that $k=4$. In this case $\mathcal{F}_{0} \cap \frac{7}{3} B_{\mathrm{M}} \subseteq\{\mu,-\mu+\lambda, \mu+\lambda, 2 \mu+\lambda, 3 \mu+\lambda\}$. It suffices then to prove that at least one of $-\mu+\lambda$ and $3 \mu+\lambda$ is not an element of $\mathcal{F}_{0}$.

Assume otherwise and set $\alpha=-\mu+\lambda, \beta=3 \mu+\lambda$. As $\Delta(\alpha, \beta)=4$, neither of these classes can be a boundary class (Proposition 2.2). Further since $\|\alpha\|_{\mathrm{M}}=\|\beta\|_{\mathrm{M}}=14$ and $H_{1}\left(M ; \mathbb{Z}_{2}\right)=\mathbb{Z}_{2}$, it follows from Proposition 9.3 that $\alpha$ and $\beta$ both have type $I$. Finally through consideration of the shape of $B_{\mathrm{M}}$, the same result implies that in fact they both have type $I(5)$.

In order to determine the $A_{\mathrm{M}}(u, v)$, we first observe that the Newton polygon $N_{\mathrm{M}}$ has vertices $(0,0),(0,2),(4,1)$ and $(4,3)$. As $(4,1)$ is a vertex of $N_{\mathrm{M}}$, Proposition 10.3 implies that $i_{*}(\lambda)=0$ and hence $i_{*}(\mu) \neq$ $0 \in H_{1}\left(M ; \mathbb{Z}_{2}\right)$. The same result now shows that there are integers $a, b$ and $\epsilon, \epsilon_{1} \in\{ \pm 1\}$ such that

$$
A_{\mathrm{M}}(u, v)=1+\left(a+b u^{2}+\epsilon_{1} u^{4}\right) v+\left(\epsilon \epsilon_{1}+\epsilon b u^{2}+\epsilon a u^{4}\right) v^{2}+\epsilon u^{4} v^{3} .
$$

According to Proposition 11.3, there is some $\theta \in\{ \pm 1\}$ and integer $j \geq 1$ for which

$$
A_{\mathrm{M}}(u, u)=\epsilon u^{7}+\epsilon a u^{6}+\epsilon_{1} u^{5}+\epsilon b u^{4}+b u^{3} \epsilon \epsilon_{1} u^{2}+a u+1
$$


is divisible by $\left(u^{4}+\theta u^{3}+u^{2}+\theta u+1\right)^{j}$. Moreover, the roots of the quotient lie in $\{ \pm 1\}$. Obviously $j=1$ and performing the division yields the quotient

$$
q(u)=\epsilon u^{3}+\epsilon(a-\theta) u^{2}+\left(\epsilon_{1}-\epsilon \theta a\right) u+\left(\epsilon b-\epsilon_{1} \theta\right)
$$

and the four identities

$$
\begin{aligned}
\epsilon b-\epsilon_{1} \theta & =1 \\
\theta b-\epsilon_{1} \theta & =1 \\
\epsilon \epsilon_{1}+\epsilon \theta-\epsilon_{1} \theta & =1 \\
\theta a-\theta \epsilon_{1}+\epsilon a & =1 .
\end{aligned}
$$

Since the roots of $q$ lie in $\{ \pm 1\}$ we have

$$
\begin{gathered}
\epsilon q(u)=u^{3}+(a-\theta) u^{2}+\left(\epsilon \epsilon_{1}-\theta a\right) u+\left(b-\epsilon \epsilon_{1} \theta\right) \\
\in\left\{u^{3}+3 u^{2}+3 u+1, u^{3}-u^{2}-u+1, u^{3}+u^{2}-u-1,\right. \\
\left.u^{3}-3 u^{2}+3 u-1\right\}
\end{gathered}
$$

and so in particular $a$ is even. Now Identity (1) implies that $b-\epsilon \epsilon_{1} \theta=\epsilon$ and so a further examination of the possible coefficients of $\epsilon q(u)$ yields $a-\theta=\epsilon\left(\epsilon \epsilon_{1}-\theta a\right)=\epsilon_{1}-\epsilon \theta a$. Hence

$$
\epsilon_{1}+\theta=(1+\epsilon \theta) a \equiv 0(\bmod 4)
$$

Thus both sides of this equation are zero. It follows that

$$
\epsilon_{1}=-\theta \text { and } a=-\epsilon \theta a
$$

Hence by Identity (2) we deduce that $b=0$. It follows from these calculations that

$$
A_{\mathrm{M}}(u, v)=1+a v-\theta u^{4} v-\epsilon \theta v^{2}-\theta a u^{4} v^{2}+\epsilon u^{4} v^{3}
$$

and consequently

$$
\begin{aligned}
A_{\mathrm{M}}\left(u, u^{-3}\right) & =1+a u^{-3}-\theta u-\epsilon \theta u^{-6}-\theta a u^{-2}+\epsilon u^{-5} \\
& =-\theta u^{-6}(u-\theta)\left(u^{6}+a u^{3}-\theta \epsilon\right) .
\end{aligned}
$$

But this contradicts Proposition 11.3 since our assumptions imply that $\beta=3 \mu+\lambda$ is an $I(5)$-type class of non-minimal norm which is not a 
boundary class. Hence at least one of $\alpha, \beta$ is not an element of $\mathcal{F}_{0}$. This completes the analysis of Subcase (II.1.b).

Subcase (II.1.c). $B_{M}$ has three pairs of vertices $\pm(3 m /(2 j+q), 3 /(2 j+$ $q)), \pm(3(m+j) /(j+q), 3 /(j+q))$ and $\pm(3(m+j+q) /(j+2 q), 3 /(j+2 q))$, for some integers $m, j>0, q>0$, with $j+q \geq 3$.

We may assume that $m=-j$. Then the vertices of $N_{\mathrm{M}}$ are $(0,1)$, $(0,2),(q, 3),(j+q, 2),(j+q, 1)$ and $(j, 0)$ (the case $j=1, q=2$ is depicted in Figure 10). Since $i^{*}(\mu) \neq 0$ (Proposition 9.6) it follows from Proposition 10.3 (5) that both $j$ and $q$ are even. One can now verify, using Proposition 9.3, that Proposition 12.2 holds.
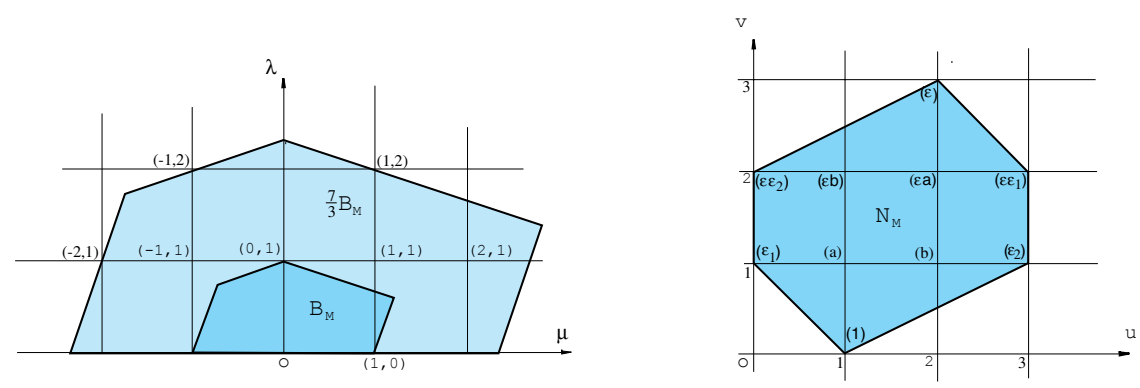

Figure 10: The canonical norm polygon and Newton polygon when $j=-1, q=2$ in Subcase II.1.c.

Subcase II.2 $\mu$ is a vertex of $B_{M}$.

According to [2, Lemma 6.6 (3)], we have seven subcases to consider.

Subcase (II.2.a). $\quad B_{M}$ is contained in a parallelogram with vertices $\pm(1,0)$ and $\pm(m, 1)$, with $(m, 1)$ being a strict boundary class associated to a vertex of $B_{M}$.

We may assume that $m=0$. Then, $\frac{7}{3} B_{M}$ is contained in the parallelogram with vertices $\pm\left(\frac{7}{3}, 0\right)$ and $\pm\left(0, \frac{7}{3}\right)$. In this parallelogram the primitive classes are $\pm(1,0), \pm(-1,1), \pm(0,1)$ and $\pm(1,1)$. Noting that $(1,0)$ and $(0,1)$ are rational multiples of the vertices of $B_{\mathrm{M}}$, and therefore strict boundary classes, we have $\# \leq 2, \Delta \leq 2$.

Subcase (II.2.b). $\quad B_{M}$ is contained in a parallelogram with vertices $\pm(1,0)$ and $\pm\left(\frac{6 m}{5}, \frac{6}{5}\right)$ with $(2 m+1,2)$ being a strict boundary class associated to a vertex of $B_{M}$.

We may assume that $m=0$. Then $\frac{7}{3} B_{M}$ is contained in the parallelogram with vertices $\pm\left(\frac{7}{3}, 0\right)$ and $\pm(0,14 / 5)$. The primitive classes in this 
parallelogram are $\pm(1,0), \pm(-1,1), \pm(0,1), \pm(1,1), \pm(2,1)$ and $\pm(1,2)$. Since $(1,0)$ and $(1,2)$ are strict boundary classes, we have $\# \leq 4, \Delta \leq 3$ and there is at most one pair of slopes realizing the distance 3 .

Subcase (II.2.c). $\quad B_{M}$ is contained in a parallelogram with vertices $\pm(1,0)$ and $\pm\left(\frac{m}{3}, 1\right)$ where $(m, 3)$ is a strict boundary class associated to a vertex of $B_{M}$.

We may assume that $m=1$. Then $\frac{7}{3} B_{M}$ is contained in the parallelogram with vertices $\pm\left(\frac{7}{3}, 0\right)$ and $\pm\left(\frac{7}{9}, \frac{7}{3}\right)$. The primitive classes in this parallelogram are $\pm(1,0), \pm(-1,1), \pm(0,1), \pm(1,1)$ and $\pm(1,2)$. Since $(1,0)$ is strict boundary class we have $\# \leq 4, \Delta \leq 3$ and there is at most one pair of slopes realizing the distance 3 .

Subcase (II.2.d). $B_{M}$ is contained in a polygon with vertices $\pm(1,0)$, $\pm(m, 1)$ and $\pm\left(\frac{3(2 m+1)}{5}, \frac{6}{5}\right)$ where $(2 m+1,2),(1,0)$ and $(m, 1)$ are strict boundary classes associated to vertices of $B_{M}$.

We may assume that $m=0$. Then $\frac{7}{3} B_{M}$ is contained in the polygon with vertices $\pm\left(\frac{7}{3}, 0\right), \pm\left(0, \frac{7}{3}\right)$ and $\pm\left(\frac{7}{5}, \frac{14}{5}\right)$. The primitive classes in this polygon are $\pm(1,0), \pm(-1,1), \pm(0,1), \pm(1,1), \pm(2,1)$ and $\pm(1,2)$. But $(1,0),(1,2)$ and $(0,1)$ are strict boundary classes, so $\# \leq 3, \Delta \leq 3$ and there is at most one pair of slopes realizing the distance 3 .

Subcase (II.2.e). $\quad B_{M}$ is contained in the polygon with vertices $\pm(1,0), \pm(m+1,1)$ and $\pm\left(\frac{3(2 m+1)}{5}, \frac{6}{5}\right)$ where $(2 m+1,2),(1,0)$ and $(m+1,1)$ are strict boundary classes associated to vertices of $B_{M}$.

We may assume that $m=0$. Then $\frac{7}{3} B_{M}$ is contained in the polygon with vertices $\pm\left(\frac{7}{3}, 0\right), \pm\left(\frac{7}{3}, \frac{7}{3}\right)$ and $\pm\left(\frac{7}{5}, \frac{14}{5}\right)$. The primitive classes in this polygon are $\pm(1,0), \pm(-1,1), \pm(0,1), \pm(1,1), \pm(2,1)$ and $\pm(1,2)$. But $(1,0),(1,2)$ and $(1,1)$ are strict boundary classes, so $\# \leq 3, \Delta \leq 3$ and there is at most one pair of slopes realizing the distance 3 .

Subcase (II.2.f). $B_{M}$ is a polygon with vertices $\pm(1,0), \pm\left(\frac{3 m}{|k|+q}, \frac{3}{|k|+q}\right)$ and $\pm\left(\frac{3(m-k)}{2|k|+q}, \frac{3}{2|k|+q}\right)$, for some integers $m, k \neq 0$ and $q>0$, satisfying $|k|+q \geq 3$.

We may assume that $m=0$ and $k<0$. Then $N_{\mathrm{M}}$ has vertices $(0,0),(0,2),(-k, 3),(q-k, 3),(q-k, 1)$ and $(q, 0)$ (the case $k=-1$ and $q=2$ is shown in Figure 11). We already know that since $\|\mu\|_{\mathrm{M}}=6$, $i^{*}(\mu) \neq 0$. Hence $q$ is even by Proposition 10.3 (5). On the other hand, from the shape of $N_{\mathrm{M}}$ we see that both even and odd powers of $v$ occur in $A_{\mathrm{M}}$, and so $i_{*}(\lambda) \neq 0$. Proposition $10.3(5)$ therefore implies that $k$ 
is odd. When $|k|+q>3$, one can easily check that Proposition 12.2 is a consequence of Proposition 9.3. Thus we must analyze the case $k=-1, q=2$.

When $k=-1$ and $q=2, B_{\mathrm{M}}$ and $N_{\mathrm{M}}$ are shown in Figure 11 and the canonical $A$-polynomial is of the form $A_{M}(u, v)=1+a u+\epsilon_{1} u^{2}+(b+$ $\left.c u+d u^{2}+\epsilon_{2} u^{3}\right) v+\left(\epsilon \epsilon_{2}+\epsilon d u+\epsilon c u^{2}+\epsilon b u^{3}\right) v^{2}+\left(\epsilon \epsilon_{1} u+\epsilon a u^{2}+\epsilon u^{3}\right) v^{3}$ for some integers $a, b, c, d$ and $\epsilon, \epsilon_{1}, e_{2} \in\{ \pm 1\}$. Since $i^{*}(\mu) \neq 0$ and $i_{*}(\lambda) \neq 0$, Proposition 10.3 (5) implies that $a=b=d=0$. Thus

$$
A_{M}(u, v)=1+\epsilon_{1} u^{2}+\left(c u+\epsilon_{2} u^{3}\right) v+\left(\epsilon \epsilon_{2}+\epsilon c u^{2}\right) v^{2}+\left(\epsilon \epsilon_{1} u+\epsilon u^{3}\right) v^{3} .
$$

The primitive classes contained in $\frac{7}{3} B_{M}$ are $\pm(1,0), \pm(-1,1), \pm(0,1)$, $\pm(1,1),(2,1)$ and $\pm(1,2)$. It suffices for us to show that $(2,1)$ is not in $\mathcal{F}_{0}$.
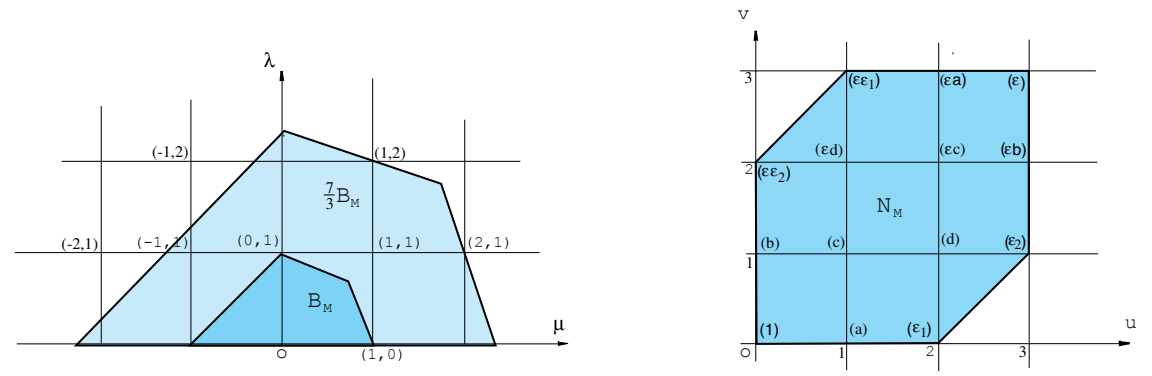

Figure 11: The canonical norm polygon and Newton polygon when $k=-1$ and $q=2$ in Subcase II.2.f.

Lemma 13.4. When $k=-1$ and $q=2, \alpha=(2,1)$ is not a finite filling class.

Proof. Suppose otherwise. From the shape of $B_{\mathrm{M}}$, we see that $\|\alpha\|_{\mathrm{M}}=s_{\mathrm{M}}+8$ and since $H_{1}\left(M ; \mathbb{Z}_{2}\right)=\mathbb{Z}_{2}, \alpha$ must be an $I(5)$-type class. Therefore by Proposition 11.3, $A_{M}\left(u, u^{-2}\right)=1+\epsilon_{1} u^{2}+(c u+$ $\left.\epsilon_{2} u^{3}\right) u^{-2}+\left(\epsilon \epsilon_{2}+\epsilon c u^{2}\right) u^{-4}+\left(\epsilon \epsilon_{1} u+\epsilon u^{3}\right) u^{-6}=u^{-5}\left(\epsilon \epsilon_{1}+\epsilon \epsilon_{2} u+\epsilon u^{2}+\right.$ $\left.\epsilon c u^{3}+c u^{4}+u^{5}+\epsilon_{2} u^{6}+\epsilon_{1} u^{7}\right)$ is divisible by either $u^{4}+u^{3}+u^{2}+u+1$ or $u^{4}-u^{3}+u^{2}-u+1$ and the roots of the quotient polynomial are \pm 1 .

Fix $\theta \in\{ \pm 1\}$. If $A_{M}\left(u, u^{-2}\right)$ is divisible by $u^{4}+\theta u^{3}+u^{2}+\theta u+1$, the quotient polynomial is $q(u)=\epsilon_{1} u^{3}+\left(\epsilon_{2}-\epsilon_{1} \theta\right) u^{2}+\left(1-\epsilon_{2} \theta\right) u+(c-\theta)$. By hypothesis $q(u)$ is congruent to $(u+1)^{3}(\bmod 2)$ and so $\epsilon_{2}-\epsilon_{1} \theta$, the coefficient of $u^{2}$ in $q$, is odd. This is clearly false. Thus $(2,1)$ is not in $\mathcal{F}_{0}$.

q.e.d. 
Subcase (II.2.g). $B_{M}$ is a polygon with vertices $\pm(1,0), \pm(3 \mathrm{~m} /(2 j+$ $k+q), 3 /(2 j+k+q)), \pm(3(m+j) /(j+k+q), 3 /(j+k+q))$ and $\pm(3(m+j+k) /(j+2 k+q), 3 /(j+2 k+q))$, for some integers $m, j>$ $0, k>0, q>0$.

We may assume that $m=-j$. Then $N_{\mathrm{M}}$ has vertices $(0,1),(0,2)$, $(k, 3),(k+q, 3),(j+k+q, 2),(j+k+q, 1),(j+q, 0)$ and $(j, 0)$. From Proposition 10.3 we see that both $j$ and $q$ are even while $k$ is odd. It now follows from Proposition 9.3 that Proposition 12.2 holds in this case. The proof of Proposition 12.2 is therefore complete. q.e.d.

\section{Proof of Proposition 12.3}

From now till the end of $\S 17$ we assume that $s_{\mathrm{M}} \geq 8$. The proof of Proposition 12.3 is based on the following three lemmas.

Lemma 14.1. Suppose that $s_{M}=8$.

(1) If $\mu$ is not a vertex of $B_{M}$, then $B_{M}$ has at most four pairs of vertices and the absolute values of the $\lambda$-coordinates of the associated boundary classes are no larger than 3. Further:

(i) If $B_{M}$ has a pair of vertices whose associated boundary classes have $\lambda$-coordinates equal to 3 in absolute value, then $B_{M}$ is a parallelogram and the absolute value of the $\lambda$-coordinate of its other pair of vertices is 1.

(ii) If $B_{M}$ has at least two pairs of vertices whose associated boundary classes have $\lambda$-coordinates which are larger than 1 in absolute value, then $B_{M}$ is a parallelogram and the absolute value of the $\lambda$-coordinate of its other pair of vertices is 2 .

(2) If $\mu$ is a vertex of $B_{M}$, then $B_{M}$ has at most five pairs of vertices and the absolute values of the $\lambda$-coordinates of the associated boundary classes are no larger than 4 . Further:

(i) If $B_{M}$ has a pair of vertices whose associated boundary classes have $\lambda$-coordinates equal to 4 in absolute value, then $B_{M}$ is parallelogram.

(ii) If $B_{M}$ has a vertex pair whose associated boundary classes have $\lambda$-coordinates equal to 3 in absolute value, then $B_{M}$ has exactly one more vertex pair, besides $\pm \mu$, and its associated boundary classes are integral. 
(iii) If $B_{M}$ has two pairs of vertices whose associated boundary slopes have $\lambda$-coordinates equal to 2 in absolute value, then $B_{M}$ has no other vertex pairs, besides $\pm \mu$.

Proof. Apply [2, Lemma 6.2].

q.e.d.

Lemma 14.2. Suppose that $s_{M}=10$.

(1) If $\mu$ is not a vertex of $B_{M}$, then $B_{M}$ has at most five pairs of vertices and the absolute values of the $\lambda$-coordinates of the associated boundary classes are no larger than 4 . Further:

(i) If $B_{M}$ has a pair of vertices whose associated boundary classes have $\lambda$-coordinates equal to 4 in absolute value, then $B_{M}$ has only one other pair of vertices and the absolute value of the $\lambda$-coordinates of its associated classes is 1 .

(ii) If $B_{M}$ has a pair of vertices whose associated boundary classes have $\lambda$-coordinates equal to 3 in absolute value, then $B_{M}$ has at most one vertex pair whose associated boundary class has $\lambda$-coordinate equal to 2 , in which case $B_{M}$ is a parallelogram.

(iii) If $B_{M}$ has a pair of vertices whose associated boundary classes have $\lambda$-coordinate equal to 3 in absolute value, then $B_{M}$ has at most two more vertex pairs whose associated boundary classes are integral.

(2) If $\mu$ is a vertex of $B_{M}$, then $B_{M}$ has at most six pairs of vertices and the absolute values of the $\lambda$-coordinates of the associated boundary classes are no larger than 5 in absolute value. Further:

(i) If $B_{M}$ has a pair of vertices whose associated boundary slopes have $\lambda$-coordinates equal to 5 in absolute value, then $B_{M}$ is parallelogram.

(ii) If $B_{M}$ has a vertex pair whose associated boundary classes have $\lambda$-coordinates equal to 4 in absolute value, then $B_{M}$ has exactly one vertex pair, besides $\pm \mu$, in which case the associated boundary class is integral.

(iii) If $B_{M}$ has a vertex pair whose associated boundary classes have $\lambda$-coordinates equal to 3 in absolute value, then $B_{M}$ has at most one vertex pair whose associated boundary slope has $\lambda$-coordinate equal to 2 in absolute value, in which case $B_{M}$ has exactly three vertex pairs. 
(iv) If $B_{M}$ has a vertex pair whose associated boundary classes have $\lambda$-coordinates equal to 3 in absolute value, then $B_{M}$ has at most two other vertex pairs whose associated boundary classes are integral.

Proof. Apply [2, Lemma 6.2].

q.e.d.

Lemma 14.3. Suppose that $s_{M}=8$. If $B_{M}$ is a parallelogram with vertices $\pm(m-1,1)$ and $\pm(m+1)$, then $\mathcal{F}_{0} \subset\{(1,0),(m, 1)\}$. In particular $\# \leq 2$ and $\Delta \leq 1$.

Proof. We may assume that $m=0$ and so $B_{\mathrm{M}}$ is as shown in Figure 12. According to Proposition 9.3, any finite filling class is contained in $\frac{5}{2} B_{M}$. Therefore $\mathcal{F}_{0} \subset\{(-1,2),(1,2),(-2,1),(2,1),(0,1),(1,0)\}$. We now use $A_{M}$ to show that $\alpha=(-2,1) \notin \mathcal{F}_{0}$. By symmetry neither are $(-1,2),(1,2)$ or $(2,1)$.

If $\alpha \in \mathcal{F}_{0}$, then since $s_{\mathrm{M}}+8=\|\alpha\|_{\mathrm{M}}>\|\alpha+\mu\|_{\mathrm{M}},\|\alpha+2 \mu\|_{\mathrm{M}},\|\alpha+3 \mu\|_{\mathrm{M}}$, Proposition 9.3 implies that it must be an $I(5)$-type class. Thus by Lemma $2.1, i_{*}(\lambda)=i_{*}(\alpha) \neq 0$.
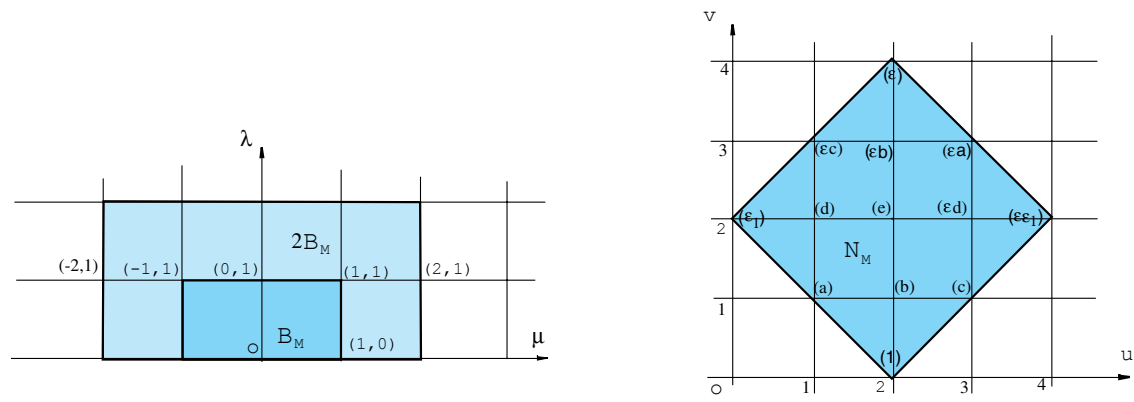

Figure 12: The canonical norm polygon and Newton polygon.

By Proposition 10.1, $N_{\mathrm{M}}$ is as shown in Figure 12. Hence the canonical $A$-polynomial is of the form $A_{M}(u, v)=u^{2}+\left(a u+b u^{2}+c u^{3}\right) v+$ $\left(\epsilon_{1}+d u+e u^{2}+\epsilon d u^{3}+\epsilon \epsilon_{1} u^{4}\right) v^{2}+\left(\epsilon c u+\epsilon b u^{2}+\epsilon a u^{3}\right) v^{3}+\epsilon u^{2} v^{4}$ for some integers $a, b, c, d, e$ and $\epsilon, \epsilon_{1} \in\{ \pm 1\}$.

By Proposition 11.3, there is some $\theta \in\{ \pm 1\}$ such that $u^{-2} A_{M}\left(u, u^{2}\right)$ $=1+a u+\left(b+\epsilon_{1}\right) u^{2}+(c+d) u^{3}+e u^{4}+\epsilon(c+d) u^{5}+\epsilon\left(b+\epsilon_{1}\right) u^{6}+\epsilon a u^{7}+\epsilon u^{8}$ is divisible by $\left(u^{4}+\theta u^{3}+u^{2}+\theta u+1\right)^{j}$ for some integer $j \geq 1$. Further the roots of the quotient lie in $\{ \pm 1\}$. Obviously $j \leq 2$ but in fact it's easy to see that $j=1$. For otherwise Proposition 11.3 implies that neither 
$A\left(u, u^{2}\right)$ nor $A\left(u,-u^{2}\right)$ have roots in $\{ \pm 1\}$. But this contradicts the fact that any discrete faithful character in $C_{\mathrm{M}}$ corresponds to a point in $\{ \pm(1,1), \pm(1,-1)\}$. Hence we have $j=1$.

Let $\epsilon$ be the nonzero element of $H_{1}\left(M ; \mathbb{Z}_{2}\right)=\mathbb{Z}_{2}$ and recall that we have shown $\epsilon(\lambda) \neq 0$. If $\epsilon(\mu)=0$ then Proposition 10.3 (5) implies that $a=b=c=0$. Thus $u^{-2} A_{M}\left(u, u^{2}\right)=1+\epsilon_{1} u^{2}+d u^{3}+e u^{4}+$ $\epsilon d u^{5}+\epsilon \epsilon_{1} u^{6}+\epsilon u^{8}$. Its quotient by $u^{4}+\theta u^{3}+u^{2}+\theta u+1$ is $q(u)=$ $\epsilon u^{4}-\epsilon \theta u^{3}+\epsilon \epsilon_{1} u^{2}+\epsilon\left(d-\epsilon_{1} \theta\right) u+(e-\epsilon \theta d)$ where $e-\epsilon \theta d=1$. But since the coefficient of $u^{3}$ in $q$ is odd, $q$ has roots other that \pm 1 , contrary to our set-up.

Assume then that $\epsilon(\mu) \neq 0$. Then Proposition 10.3 (5) implies that $b=d=0$. Calculation shows that up to a power of $u$ we have

$$
A_{\mathrm{M}}\left(u, u^{2}\right)=\epsilon u^{8}+\epsilon a u^{7}+\epsilon \epsilon_{1} u^{6}+\epsilon c u^{5}+e u^{4}+c u^{3}+\epsilon_{1} u^{2}+a u+1 .
$$

Dividing $A_{\mathrm{M}}\left(u, u^{2}\right)$ by $u^{4}+\theta u^{3}+u^{2}+\theta u+1$ yields the quotient polynomial

$$
q(u)=\epsilon u^{4}+\epsilon(a-\theta) u^{3}+\epsilon\left(\epsilon_{1}-\theta a\right) u^{2}+\epsilon\left(c-\theta \epsilon_{1}\right) u+1 .
$$

Since the roots of $q$ lie in $\{ \pm 1\}$, examination of its coefficients shows that both $a$ and $c$ are odd. Hence by Proposition 10.3 (4) we see that $|a|=|c|=1$. The same result then implies that $\epsilon_{1}=\epsilon=1$. Hence the leading and constant coefficients of $q$ are both 1 . It follows that $q(u)$ is either $(u+1)^{4},(u-1)^{4}$, or $(u+1)^{2}(u-1)^{2}$. Since $|a|=1$ we have $|a-\theta| \leq 2$ and thus $q(u)=(u+1)^{2}(u-1)^{2}=u^{4}-2 u^{2}+1$. Examination of the coefficient of $u^{3}$ in $q$ implies that $a=\theta$. But then from the coefficient of $u^{2}$ we obtain $-2=\epsilon\left(\epsilon_{1}-\theta a\right)=1-1=0$. This final contradiction completes the proof of Lemma 14.3.

q.e.d.

We are now ready to give the proof of Proposition 12.3. For points $v_{1}, v_{2} \in H_{1}(\partial M ; \mathbb{R})$, let $\left[v_{1}, v_{2}\right]$ denote the line segment they span.

Assume that there are two classes in $\mathcal{F}_{0}$ whose $\lambda$-coordinate is 2 . By [2, Theorem 1.1(1)], there are at most two such classes and if two, we may assume without loss of generality that they are $\pm(1,2)$ and $\pm(-1,2)$. By Proposition 9.3 (1) and (2), neither of these two slopes has type $C, D, Q, T(2), O(2)$ or $I(2)$.

Assume first that $\|(-1,2)\|_{\mathrm{M}},\|(1,2)\|_{\mathrm{M}} \leq 2 s_{\mathrm{M}}$. Then $[(-1 / 2,1)$, $(1 / 2,1)] \subset B_{\mathrm{M}}$. Since this segment contains $(0,1)$ it follows that it actually lies on $\partial B_{\mathrm{M}}$. Noting that $(0,1)$ is not a vertex of $B_{\mathrm{M}}$, our conventions imply that neither is $(1,0)$. Hence if $s_{\mathrm{M}}=8$, Lemma 14.1 implies 
that $B_{\mathrm{M}}$ must be a parallelogram with vertices $\pm(-1,1), \pm(1,1)$. But then from Lemma 14.3 we deduce that $(-1,2) \notin \mathcal{F}_{0}$. Thus $s_{\mathrm{M}}>8$ and since $\|(-1,2)\|_{\mathrm{M}}=\|(1,2)\|_{\mathrm{M}}=2 s_{\mathrm{M}}$, it follows from Proposition 9.3 that $(-1,2)$ and $(1,2)$ are both of type $O(3)$. Now $\Delta((1,2),(-1,1))=3$, and so from Proposition 9.3 we see that $2 s_{\mathrm{M}}=\|(1,2)\|_{\mathrm{M}} \leq\|(-1,1)\|_{\mathrm{M}}$. But this contradicts the fact that $(-2 / 3,2 / 3) \in[(-1 / 2,1),(-1,0)] \subset B_{\mathrm{M}}$ and therefore $\|(-1,1)\|_{\mathrm{M}}=\frac{3}{2}\|(-2 / 3,2 / 3)\|_{\mathrm{M}}<2 s_{\mathrm{M}}$. Thus one of $\|(-1,2)\|_{\mathrm{M}}$ and $\|(1,2)\|_{\mathrm{M}}$ is larger than $2 s_{\mathrm{M}}$.

By symmetry we may suppose that $\alpha=\|(1,2)\|_{\mathrm{M}}>2 s_{\mathrm{M}}$. From Proposition 9.3 we see that it must be an $O(3)$-type filling class and so as above we have $2 s_{M}<\|(1,2)\|_{M} \leq\|(-1,1)\|_{M}$. Therefore $\|(-1 / 2,1 / 2)\|_{M}$ $>s_{M}$. Now $(-1 / 2,1 / 2) \in[(-1,0),(0,1)]$ and since $\|(-1,0)\|_{\mathrm{M}}=s_{\mathrm{M}}$, the convexity of $\|\cdot\|_{\mathrm{M}}$ implies that $\|v\|_{\mathrm{M}}>s_{\mathrm{M}}$ for each $v \in[(-1 / 2,1 / 2),(0,1)]$. In particular we have $\|(-1 / 3,2 / 3)\|_{\mathrm{M}}>s_{\mathrm{M}}$. Thus $\|(-1,2)\|_{\mathrm{M}} \geq 3 s_{\mathrm{M}} \geq$ $s_{\mathrm{M}}+16$, which contradicts Proposition 9.3 (5). The proof of Proposition 12.3 is therefore complete.

q.e.d.

\section{Proof of Proposition 12.4}

Let $\mathcal{I}_{0}$ be the set of integral classes in $L$ which are finite or cyclic filling classes, but not strict boundary classes, and let $\Delta\left(\mathcal{I}_{0}\right)$ denote the maximal distance between elements in $\mathcal{I}_{0}$. We only need to show that $\Delta\left(\mathcal{I}_{0}\right) \leq 3$ (which implies that the number of elements in $\mathcal{I}_{0}$ is at most $4)$.

Fix $\alpha_{1} \in \mathcal{I}_{0}$ which satisfies

$$
\|\alpha\|_{\mathrm{M}} \leq\left\|\alpha_{1}\right\|_{\mathrm{M}} \text { for each } \alpha \in \mathcal{I}_{0} .
$$

As a first case, suppose that $\left\|\alpha_{1}\right\|_{\mathrm{M}}<2 s_{\mathrm{M}}$. Then $\mathcal{I}_{0} \subset \operatorname{int}\left(2 B_{\mathrm{M}}\right)$. Since each horizontal line in the $\mu \lambda$-plane intersects $B_{\mathrm{M}}$ in a line segment of length no larger than 2 , there exists a line segment $L \subset\{(u, 1) \mid u \in \mathbb{R}\}$ of length strictly less than 4 which contains $\mathcal{I}_{0}$. Thus Proposition 12.4 holds in this case.

We shall therefore assume below that $\left\|\alpha_{1}\right\|_{\mathrm{M}} \geq 2 s_{\mathrm{M}}$. Since $s_{\mathrm{M}} \geq 8$, Proposition 9.3 implies that we may assume one of the following cases holds:

- $\alpha_{1}$ is of type $D, Q, I(q)$ or $O(q)$ where $q \leq 3$.

- $s_{\mathrm{M}}=8,\left\|\alpha_{1}\right\|_{\mathrm{M}}=16$, and each $\alpha \in \mathcal{I}_{0}$ with $\|\alpha\|_{\mathrm{M}}=16$ is an $I(5)$-type class. 
We consider these two cases separately below.

Case 1. $\alpha_{1}$ is of type $D, Q, I(q)$ or $O(q)$ where $q \leq 3$.

Suppose first of all that there is an integral class $\alpha_{0}$ in $L$ such that $\left\|\alpha_{0}\right\|_{\mathrm{M}}<\left\|\alpha_{1}\right\|_{\mathrm{M}}$. Then there is an integer $j \neq 0$ such that $\alpha_{0}=\alpha_{1}+j \mu$. Set $\epsilon=j /|j|$ and choose integers $m, r \geq 0$ such that $r<q$ and $|j|=m d+$ $r$. Then by the choice of $q$ we have $\left\|\alpha_{1}+\epsilon m q \mu\right\|_{\mathrm{M}},\left\|\alpha_{1}+\epsilon(m+1) q \mu\right\|_{\mathrm{M}} \geq$ $\left\|\alpha_{1}\right\|_{\mathrm{M}}>\left\|\alpha_{0}\right\|_{\mathrm{M}}=\left\|\alpha_{1}+\epsilon(m q+r) \mu\right\|_{\mathrm{M}}$. By the properties of a norm we must have $\left\|\alpha_{1}+\epsilon k \mu\right\|_{\mathrm{M}}>\left\|\alpha_{1}\right\|_{\mathrm{M}}$ for each $k<m q$ and each $k>(m+1) q$. But this implies that $m=0$ and $\mathcal{I}_{0} \subset\left\{\alpha_{1}, \alpha_{1}+\epsilon \mu, \ldots, \alpha_{1}+q \epsilon \mu\right\}$. Therefore Proposition 12.4 holds.

Now assume that $\left\|\alpha_{1}\right\|_{\mathrm{M}} \leq\|\alpha\|_{\mathrm{M}}$ for each integral class $\alpha \in L$. By the choice of $\alpha_{1}$ it follows that $\|\alpha\|_{\mathrm{M}}=\left\|\alpha_{1}\right\|_{\mathrm{M}}$ for each $\alpha \in \mathcal{I}_{0}$. Without loss of generality we may assume that $\mathcal{I}_{0} \subset\left\{\alpha_{1}, \alpha_{1}+\mu, \ldots, \alpha_{1}+k \mu\right\}$. Clearly $\left\|\alpha_{1}+j \mu\right\|_{\mathrm{M}}=\left\|\alpha_{1}\right\|_{\mathrm{M}}$ for $0 \leq j \leq k$. Since $\Delta\left(\mathcal{I}_{0}\right)=k$, we need to show that $k \leq 3$.

If $\left\|\alpha_{1}\right\|_{\mathrm{M}}=2 s_{\mathrm{M}}$ and $k \geq 4$, then $\frac{\alpha_{1}}{2}, \frac{\alpha_{1}}{2}+\mu$ and $\frac{\alpha_{1}}{2}+2 \mu$ all lie on $\partial B_{\mathrm{M}}$. It follows that $B_{\mathrm{M}}$ is a parallelogram with vertices $\frac{\alpha_{1}}{2}$ and $\frac{\alpha_{1}}{2}+2 \mu$. But then $\alpha_{1}$ is a strict boundary class, contrary to our hypotheses. Thus we must have $k \leq 3$ when $\left\|\alpha_{1}\right\|_{\mathrm{M}}=2 s_{\mathrm{M}}$, so Proposition 12.4 holds in this case.

We may suppose then, for the rest of the proof of Case 1, that $\left\|\alpha_{1}\right\|_{\mathrm{M}}>2 s_{\mathrm{M}}$. From Proposition 9.3 , we see that $s_{\mathrm{M}}=8$ or 10 , each $\alpha \in \mathcal{I}_{0}$ is an $O$-type class, $\left\|\alpha_{1}\right\|_{\mathrm{M}} \leq s_{\mathrm{M}}+12$, and $H_{1}\left(M ; \mathbb{Z}_{2}\right) \cong \mathbb{Z}_{2} \oplus \mathbb{Z}_{2}$.

If $s_{\mathrm{M}}=10$ then by Proposition $9.6, \mu \notin \operatorname{ker}\left(H_{1}\left(\partial M ; \mathbb{Z}_{2}\right) \rightarrow H_{1}(M ;\right.$ $\left.\mathbb{Z}_{2}\right)$ ) and so one of $\left\|\alpha_{1}\right\|_{\mathrm{M}}$ and $\left\|\alpha_{1}+\mu\right\|_{\mathrm{M}}$ is divisible by 4 . But then since $20=2 s_{\mathrm{M}}<\left\|\alpha_{1}\right\|_{\mathrm{M}}<s_{\mathrm{M}}+12=22,\left\|\alpha_{1}+\mu\right\|_{\mathrm{M}} \neq\left\|\alpha_{1}\right\|_{\mathrm{M}}$. Hence $k=0$.

Last we consider the case where $s_{\mathrm{M}}=8$. As $2 \alpha_{1} \in \operatorname{ker}\left(H_{1}\left(\partial M ; \mathbb{Z}_{2}\right)\right.$ $\left.\rightarrow H_{1}\left(M ; \mathbb{Z}_{2}\right)\right),\left\|2 \alpha_{1}\right\|_{\mathrm{M}}$ is divisible by 8 by Proposition 9.6 and thus $\left\|\alpha_{1}\right\|_{\mathrm{M}}$ is visible by 4 . But $16=2 s_{\mathrm{M}}<\left\|\alpha_{1}\right\|_{\mathrm{M}} \leq s_{\mathrm{M}}+12=20$ and so $\left\|\alpha_{1}\right\|_{\mathrm{M}}=20$. Hence $\mathcal{I}_{0} \subset \partial\left(\frac{5}{2} B_{\mathrm{M}}\right)$. Since neither $\alpha_{1}$ nor $\alpha_{1}+k \mu$ are strict boundary slopes we must have $k \leq 4$ (cf. the argument for the case $\left.\left\|\alpha_{1}\right\|_{\mathrm{M}}=2 s_{\mathrm{M}}\right)$.

Suppose $k=4$. Then $\frac{2}{5} \alpha_{1}, \frac{2}{5}\left(\alpha_{1}+2 \mu\right)$ and $\frac{2}{5}\left(\alpha_{1}+4 \mu\right)$ lie on $\partial B_{\mathrm{M}}$ but are not vertices of $B_{\mathrm{M}}$. By Lemma 14.1, we see that $B_{\mathrm{M}}$ is a parallelogram with vertices $\left\{ \pm \frac{2}{5}\left(2 \alpha_{1}-\mu\right), \pm \frac{2}{5}\left(2 \alpha_{1}+9 \mu\right)\right\}$. Without loss of generality we may take $\lambda=\alpha_{1}+2 \mu$. Then the Newton polygon $N_{\mathrm{M}}$ is a diamond with vertices $(5,0),(0,2),(5,4)$ and $(10,2)$. Therefore the canonical polynomial $A_{\mathrm{M}}(u, v)$ has $u^{5}$ (up to sign) as a monomial. Now 
we must have $\mu \in \operatorname{ker}\left(H_{1}\left(\partial M ; \mathbb{Z}_{2}\right) \rightarrow H_{1}\left(M ; \mathbb{Z}_{2}\right)\right)$ since the monomial $u^{5}$ appears in $A_{\mathrm{M}}(u, v)$ (by Proposition 10.3 (5) (i) (iii)). But then by Proposition 9.6 (2), we would have $\|\mu\|_{\mathrm{M}} \geq 16$ (note that $\mu$ is not a vertex of $B_{\mathrm{M}}$ ). This contradiction completes the proof of Proposition 12.4 in Case 1.

Case 2. $s_{\mathrm{M}}=8,\left\|\alpha_{1}\right\|_{\mathrm{M}}=16$, and each $\alpha \in \mathcal{I}_{0}$ with $\|\alpha\|_{\mathrm{M}}=16$ is an $I(5)$-type class.

Choose $\alpha_{0} \in \mathcal{I}_{0}$ and $k \geq 0$ so that $\alpha_{0}+k \mu \in \mathcal{I}_{0}$ and $\mathcal{I}_{0} \subset\left\{\alpha_{0}, \alpha_{0}+\right.$ $\left.\mu, \ldots, \alpha_{0}+k \mu\right\}$. We must show that $k \leq 3$.

Since $\mathcal{I}_{0} \subset 2 B_{\mathrm{M}}$ we have $k \leq 4$. If $k=4$ then the horizontal line segment of length $2,\left[\frac{\alpha_{0}}{2}, \frac{\alpha_{0}}{2}+2 \mu\right]$, lies in $B_{\mathrm{M}}$. This in particular implies that $\mu$ is not a vertex of $B_{\mathrm{M}}$. Since neither $\alpha_{0}$ nor $\alpha_{0}+4 \mu$ are strict boundary classes, Lemma 14.1 implies that $B_{\mathrm{M}}$ is a parallelogram with vertices $\left\{ \pm\left(\alpha_{0}+\mu, 1\right), \pm\left(\alpha_{0}+3 \mu, 1\right)\right\}$, or $\left\{ \pm \frac{1}{3}\left(2 \alpha_{0}+\mu\right), \pm \frac{1}{3}\left(2 \alpha_{0}+7 \mu\right)\right\}$, or $\left\{ \pm \frac{1}{4}\left(3 \alpha_{0}+2 \mu\right), \pm\left(\alpha_{0}+3 \mu\right)\right\}$, or $\left\{ \pm\left(\alpha_{0}+\mu\right), \pm \frac{1}{4}\left(3 \alpha_{0}+10 \mu\right)\right\}$. Now the latter two cases are not possible because for these configurations we have $8=\left\|\frac{7}{8}\left(\alpha_{0}+2 \mu\right)\right\|_{\mathrm{M}}$ and so $\left\|\alpha_{0}+2 \mu\right\|_{\mathrm{M}} \notin \mathbb{Z}$. By Lemma 14.3 , the first case cannot occur. Hence $B_{\mathrm{M}}$ must have vertices $\left\{ \pm \frac{1}{3}\left(2 \alpha_{0}+\mu\right), \pm \frac{1}{3}\left(2 \alpha_{0}+\right.\right.$ $7 \mu)\}$. In particular, $\left\|\alpha_{0}\right\|_{\mathrm{M}}=\left\|\alpha_{0}+4 \mu\right\|_{\mathrm{M}}=16$ and thus both of $\alpha_{0}$ and $\alpha_{0}+4 \mu$ are $I(5)$-type filling classes. The proof of Proposition 12.4 in Case 2 is completed by an appeal to the following lemma.

Lemma 15.1. Suppose that $s_{M}=8$ and $B_{M}$ is a parallelogram with vertices $\left\{ \pm \frac{1}{3}\left(2 \alpha_{0}+\mu\right), \pm \frac{1}{3}\left(2 \alpha_{0}+7 \mu\right)\right\}$, where $\alpha_{0}$ is an integral class in L. If $\alpha_{0}$ is an I(5)-type class, then $\alpha_{0}+4 \mu$ is not an I(5)-type class.

Proof. Without loss of generality we may take $\lambda=\alpha_{0}+2 \mu$. Then $B_{\mathrm{M}}$ is the polygon with vertices $\pm \frac{2}{3}(-3,2), \pm \frac{2}{3}(3,2)$ and therefore $N_{\mathrm{M}}$ is the diamond with vertices $(3,0),(0,2),(3,4)$ and $(6,2)$. It follows from Proposition 10.3 that $i_{*}(\mu)=0$. The same result now implies that there are integers $a, b, c$ and $\epsilon, \epsilon_{1} \in\{ \pm 1\}$ such that $A_{\mathrm{M}}(u, v)=$ $u^{3}+\left(\epsilon_{1}+a u+b u^{2}+c u^{3}+\epsilon b u^{4}+\epsilon a u^{5}+\epsilon \epsilon_{1} u^{6}\right) v^{2}+\epsilon u^{3} v^{4}$ where $c=\epsilon c$. Setting $v=u^{2}$ we obtain

$$
A_{\mathrm{M}}\left(u, u^{2}\right)=u^{3}\left[\epsilon u^{8}+\epsilon \epsilon_{1} u^{7}+\epsilon a u^{6}+\epsilon b u^{5}+c u^{4}+b u^{3}+a u^{2}+\epsilon_{1} u+1\right] .
$$

Suppose that $\alpha_{0}=(-2,1)$ is an $I(5)$-type class. Then by Proposition 11.3 there is a $\theta \in\{ \pm 1\}$ and $d \geq 1$ for which $A_{\mathrm{M}}\left(u, u^{2}\right)$ is divisible by $\left(u^{4}+\theta u^{3}+u^{2}+\theta u+1\right)^{j}$. As in Lemma 14.3, we see that $j=1$. Long division of $u^{-3} A_{\mathrm{M}}\left(u, u^{2}\right)$ by $\left(u^{4}+\theta u^{3}+u^{2}+\theta u+1\right)$ yields the quotient

$$
q(u)=\epsilon u^{4}+\epsilon\left(\epsilon_{1}-\theta\right) u^{3}+\epsilon\left(a-\theta \epsilon_{1}\right) u^{2}+\epsilon(b-\theta a) u+1
$$


as well as the equation $c-\theta \epsilon b=1$. Since the roots of $q$ are \pm 1 and the coefficient of $u^{3}$ is $\epsilon\left(\epsilon_{1}-\theta\right) \in\{-2,0,2\}$, it follows that $\epsilon q(u)=$ $(u-1)^{3}(u+1),(u-1)^{2}(u+1)^{2}$ or $(u-1)(u+1)^{3}$. It can then be argued that

$$
a=-1, b=-\epsilon \theta, c=0, \epsilon_{1}=\epsilon \theta .
$$

Thus

$$
A_{\mathrm{M}}(u, v)=u^{3}+\left(\epsilon \theta-u-\epsilon \theta u^{2}-\theta u^{4}-\epsilon u^{5}+\theta u^{6}\right) v^{2}+\epsilon u^{3} v^{4} .
$$

If we assume that $\alpha_{0}+4 \mu=2 \mu+\lambda$ is also an $I(5)$-type class then

$$
u^{5} A_{\mathrm{M}}\left(u, u^{-2}\right)=u^{8}+\theta u^{7}-\epsilon u^{6}-\theta u^{5}-\epsilon \theta u^{3}-u^{2}+\epsilon \theta u+\epsilon
$$

has either $\zeta=e^{\frac{2 \pi i}{5}}$ or $-\zeta$ as a root. Substituting these values into $A_{\mathrm{M}}\left(u, u^{-2}\right)$ yields $\epsilon=1$ and

$$
\theta=\left\{\begin{aligned}
1 & \text { if } \zeta \text { is a root of } A_{\mathrm{M}}\left(u, u^{-2}\right) \\
-1 & \text { if }-\zeta \text { is a root of } A_{\mathrm{M}}\left(u, u^{-2}\right)
\end{aligned}\right.
$$

Thus

$$
A_{\mathrm{M}}(u, v)=u^{3}+\left(\theta-u-\theta u^{2}-\theta u^{4}-u^{5}+\theta u^{6}\right) v^{2}+u^{3} v^{4} .
$$

But it is proven in the appendix to this paper that there is no compact, irreducible, orientable, hyperbolic 3-manifold $M$ whose boundary is a torus which has such a canonical $A$-polynomial. Thus $\alpha_{0}+4 \mu$ is not an $I(5)$-type class.

q.e.d.

\section{Proof of Proposition 12.5}

Suppose that $\alpha, \beta \in \mathcal{F}_{0}$. We need to show that $\Delta(\alpha, \beta) \leq 3$. Suppose otherwise. Then by Propositions 12.3 and 12.4 we see that one of $\alpha$ and $\beta$, say $\beta$, has $\lambda$-coordinate 2 , while $\alpha$ is integral. After possibly changing $\lambda$ we can assume that $\beta=(1,2)$. Since the distance between an integral class and $\beta$ is always an odd number, [2, Theorem 1.1(1)] implies that $\Delta(\alpha, \beta)=5$. Hence $\alpha=(-2,1)$ or $(3,1)$ and by possibly changing the orientation of $\lambda$ we may assume that $\alpha=(-2,1)$. Let $\gamma$ and $\eta$ denote $(-1,1)$ and $(1,1)$ respectively.

An application of [2, Theorem 1.1 (2)] yields the fact that neither $\alpha$ nor $\beta$ is a cyclic filling class.

Recall that for points $\omega, \sigma \in V=H_{1}(\partial M ; \mathbb{R})$, we use $[\omega, \sigma]$ to denote the line segment in $V$ with endpoints $\omega$ and $\sigma$.

The following lemma records several useful inequalities. 


\section{Lemma 16.1.}

(1) $\|\lambda\|_{M},\|\eta\|_{M}<\|\beta\|_{M}$.

(2) (i) $5\|\lambda\|_{M} \leq\|\alpha\|_{M}+2\|\beta\|_{M}$ with equality if and only if $\left[\frac{s_{M}}{\|\alpha\|_{M}} \alpha\right.$, $\left.\frac{s_{M}}{\|\beta\|_{M}} \beta\right] \subset \partial B_{M}$.

(ii) If $5 s_{M}=\|\alpha\|_{M}+2\|\beta\|_{M}$, then $\lambda \in \partial B_{M}$.

(3) $\|\beta\|_{M} \leq\|\alpha\|_{M}$ and $\|\beta\|_{M}=\|\alpha\|_{M}$ if and only if both $\alpha$ and $\beta$ are of type $I(5)$.

Proof. (1) Suppose that $\|\beta\|_{\mathrm{M}} \leq\|\lambda\|_{\mathrm{M}}$. Then since $\beta=2 \lambda+\mu$ we have $\|\beta\|_{\mathrm{M}}=\|2 \lambda+\mu\|_{\mathrm{M}} \geq 2\|\lambda\|_{\mathrm{M}}-s_{\mathrm{M}} \geq 2\|\beta\|_{\mathrm{M}}-s_{\mathrm{M}}$ and so $\|\beta\|_{\mathrm{M}} \leq s_{\mathrm{M}}$ which contradicts Propsition 4.5 (3). Thus $\|\beta\|_{\mathrm{M}}>\|\lambda\|_{\mathrm{M}}$ and a similar argument shows that $\|\beta\|_{\mathrm{M}}>\|\eta\|_{\mathrm{M}}$.

(2) (i) The line segment $\left[\frac{s_{\mathrm{M}}}{\|\alpha\|_{\mathrm{M}}} \alpha, \frac{s_{\mathrm{M}}}{\|\beta\|_{\mathrm{M}}} \beta\right]$ lies entirely in $B_{\mathrm{M}}$ and intersects the $\lambda$-axis at $\left(0, \frac{5 s_{\mathrm{M}}}{\|\alpha\|_{\mathrm{M}}+2\|\beta\|_{\mathrm{M}}}\right)$. On the other hand $\partial B_{\mathrm{M}}$ intersects the positive $\lambda$-axis at $\left(0, \frac{s_{\mathrm{M}}}{\|\lambda\|_{\mathrm{M}}}\right)$ and so $\frac{5 s_{\mathrm{M}}}{\|\alpha\|_{\mathrm{M}}+2\|\beta\|_{\mathrm{M}}} \leq \frac{s_{\mathrm{M}}}{\|\lambda\|_{\mathrm{M}}}$, i.e., $5\|\lambda\|_{\mathrm{M}} \leq\|\alpha\|_{\mathrm{M}}+2\|\beta\|_{\mathrm{M}}$. Further if there is equality then the three points $\frac{s_{\mathrm{M}}}{\|\alpha\|_{\mathrm{M}}} \alpha, \frac{s_{\mathrm{M}}}{\|\lambda\| \mathrm{M}} \lambda, \frac{s_{\mathrm{M}}}{\|\beta\|_{\mathrm{M}}} \beta$ of norm $s_{\mathrm{M}}$ all lie on $\left[\frac{s_{\mathrm{M}}}{\|\alpha\|_{\mathrm{M}}} \alpha, \frac{s_{\mathrm{M}}}{\|\beta\|_{\mathrm{M}}} \beta\right]$. Thus $\left[\frac{s_{\mathrm{M}}}{\|\alpha\|_{\mathrm{M}}} \alpha, \frac{s_{\mathrm{M}}}{\|\beta\|_{\mathrm{M}}} \beta\right] \subset \partial B_{\mathrm{M}}$.

(ii) If $5 s_{\mathrm{M}}=\|\alpha\|_{\mathrm{M}}+2\|\beta\|_{\mathrm{M}}$, then part (i) shows that $s_{\mathrm{M}}=\|\lambda\|_{\mathrm{M}}$.

(3) According to part (1) of this lemma we have $\|\beta\|>s_{\mathrm{M}}$ and so by Proposition 9.3, $\beta$ cannot have type $C, D, Q$, or $T(q), I(q), O(q)$ where $q \leq 2$.

Assume next that $\beta$ is of type $T(3), O(3)$ or $I(3)$. Recall $\gamma=(-1,1)$. Then since $\Delta(\beta, \gamma)=3$, Proposition 9.3 implies $\|\beta\|_{\mathrm{M}} \leq\|\gamma\|_{\mathrm{M}}$. There are some $x, t \in(0,1)$ such that $\gamma=x((1-t) \alpha+t \beta)$ and so $\|\gamma\|_{\mathrm{M}}<$ $\|(1-t) \alpha+t \beta\|_{\mathrm{M}} \leq(1-t)\|\alpha\|_{\mathrm{M}}+t\|\beta\|_{\mathrm{M}} \leq(1-t)\|\alpha\|_{\mathrm{M}}+t\|\gamma\|_{\mathrm{M}}$. Hence $\|\alpha\|_{\mathrm{M}}>\|\gamma\|_{\mathrm{M}} \geq\|\beta\|_{\mathrm{M}}$.

If $\beta$ is of type $I(5)$, then since $\Delta(\beta, \alpha)=5$, Proposition 9.3 (4) shows that $\alpha$ is also of type $I(5)$ and $\|\beta\|_{\mathrm{M}}=\|\alpha\|_{\mathrm{M}}$. Hence part (3) of the lemma holds in this case.

Next we suppose that $\beta$ is of type $O(4)$ and that $\|\alpha\|_{\mathrm{M}}<\|\beta\|_{\mathrm{M}}$. According to Proposition $9.3(5),\|\beta\|_{\mathrm{M}} \leq s_{\mathrm{M}}+6$ and so by part (2) we have $5 s_{\mathrm{M}} \leq 5\|\lambda\|_{\mathrm{M}} \leq\|\alpha\|_{\mathrm{M}}+2\|\beta\|_{\mathrm{M}} \leq 3 s_{\mathrm{M}}+16$. Thus $s_{\mathrm{M}}=8,\|\alpha\|_{\mathrm{M}}=$ $s_{\mathrm{M}}+4,\|\beta\|_{\mathrm{M}}=s_{\mathrm{M}}+6$ from part (2) we obtain $\|\lambda\|_{\mathrm{M}}=s_{\mathrm{M}}$ and the line segment $\left[\frac{s_{\mathrm{M}}}{\|\alpha\|_{\mathrm{M}}} \alpha, \frac{s_{\mathrm{M}}}{\|\beta\|_{\mathrm{M}}} \beta\right]=\left[\frac{2}{3} \alpha, \frac{4}{7} \beta\right]$ lies in $\partial B_{\mathrm{M}}$. Now the segment $\left[\mu, \frac{4}{7} \beta\right]$ also lies in $B_{\mathrm{M}}$ and intersects the horizontal line through $\frac{2}{3} \alpha \in \partial B_{\mathrm{M}}$ in 
the point $\left(\frac{3}{4}, \frac{2}{3}\right)=\frac{2}{3} \alpha+\frac{25}{12} \mu$. But this is impossible as $B_{\mathrm{M}}$ contains no horizontal segment of length larger than 2 .

Finally suppose that $\beta$ is of type $O(4)$ and that $\|\alpha\|_{\mathrm{M}}=\|\beta\|_{\mathrm{M}}$. We must show that this case cannot occur. The inequalities of part (2) imply that $s_{\mathrm{M}}=8,\|\lambda\|_{\mathrm{M}}=s_{\mathrm{M}}$ and $\|\alpha\|_{\mathrm{M}}=\|\beta\|_{\mathrm{M}}=s_{\mathrm{M}}+6=\frac{7}{4} s_{\mathrm{M}}$. Hence by Proposition 9.3 (3) and (5), we see that $\alpha$ is not of type $T$. As $\beta$ is of type $O(4)$ we have $H_{1}\left(M ; \mathbb{Z}_{2}\right)=\mathbb{Z}_{2}$ and therefore by Lemma 2.1 $i_{*}(\mu)=i_{*}(\beta)=0$. It follows that $i_{*}(\alpha) \neq 0$ and so $H_{1}\left(M(\alpha) ; \mathbb{Z}_{2}\right)=0$. Hence Lemma 2.1 implies that $\alpha$ must be of type $I$ (we already knew that it is not of type $C$ or $T$ ). Now $\alpha$ cannot be of type $I(2)$ since $\|\alpha\|_{\mathrm{M}}>s_{\mathrm{M}}=\|\lambda\|_{\mathrm{M}}$. It cannot have type $I(5)$ for otherwise $\beta$ would also (Proposition $9.3(4)$ ), so as our final case assume it is of type $I(3)$. Then since $\Delta(\alpha, \eta)=3$ we have $\|\eta\|_{\mathrm{M}} \geq\|\alpha\|_{\mathrm{M}}=s_{\mathrm{M}}+6$ and therefore $\left(\frac{4}{7}, \frac{4}{7}\right)$ is not contained in the interior of $B_{\mathrm{M}}$. On the other hand, we have $\|\beta\|_{\mathrm{M}}=s_{\mathrm{M}}+6$ and thus the line segment $\left[\left(\frac{4}{7}, \frac{8}{7}\right), \mu\right]$ is contained in $B_{\mathrm{M}}$. But the segments $\left[\left(\frac{4}{7}, \frac{8}{7}\right), \mu\right]$ and $[\eta,-\eta]$ intersect in the point $\left(\frac{8}{11}, \frac{8}{11}\right)$, which implies that $\left(\frac{4}{7}, \frac{4}{7}\right)$ is contained in the interior of $B_{\mathrm{M}}$. This contradiction completes the proof of the lemma.

We now complete the proof of Proposition 12.5.

Lemma 16.2. $\alpha$ cannot be of type $C, D, Q$ or $O(q), T(q), I(q)$ where $q \leq 3$.

Proof. This follows from Proposition 9.3 since $\|\alpha\|_{\mathrm{M}} \geq\|\beta\|_{\mathrm{M}}>$ $\|\lambda\|_{\mathrm{M}},\|\eta\|_{\mathrm{M}} \geq s_{\mathrm{M}}$ by Lemma 16.1. q.e.d.

Lemma 16.3. $\alpha$ cannot be of type $O(4)$.

Proof. If $\alpha$ has type $O(4)$ then from Proposition 9.3 (5) and Lemma 16.1 (3) we see that $\|\beta\|_{\mathrm{M}}<\|\alpha\|_{\mathrm{M}} \leq s+6$. Hence by Lemma 16.1 (2) we have $5 s_{\mathrm{M}} \leq 3 s_{\mathrm{M}}+14$. But then $s_{\mathrm{M}}<8$, a contradiction. Therefore the lemma holds. q.e.d.

Lemma 16.4. $\alpha$ cannot be of type $I(5)$.

Proof. If $\alpha$ is of type $I(5)$, then from Proposition 9.3 (4) we see that $\beta$ also has type $I(5)$ and $\|\alpha\|_{\mathrm{M}}=\|\beta\|_{\mathrm{M}}$. Hence Lemma 2.1 implies that $i_{*}(\mu)=i_{*}(\beta)=i_{*}(\alpha) \neq 0$ while $i_{*}(\gamma)=i_{*}(\eta)=0$. Then by Proposition 9.6, both $\|\gamma\|_{\mathrm{M}}$ and $\|\eta\|_{\mathrm{M}}$ are divisible by 4 . Since $\|\alpha\|_{\mathrm{M}}=$ $\|\beta\|_{\mathrm{M}} \leq s_{\mathrm{M}}+8$, Lemma 16.1 (2) shows that one of the following cases holds: 
(i) $s_{\mathrm{M}}=12=\|\lambda\|_{\mathrm{M}},\|\alpha\|_{\mathrm{M}}=\|\beta\|_{\mathrm{M}}=s_{\mathrm{M}}+8$, and $\left[\frac{s_{\mathrm{M}}}{\|\alpha\|_{\mathrm{M}}} \alpha, \frac{s_{\mathrm{M}}}{\|\beta\|_{\mathrm{M}}} \beta\right] \subset$ $\partial B_{\mathrm{M}}$.

(ii) $s_{\mathrm{M}}=10=\|\lambda\|_{\mathrm{M}}$ and $\|\alpha\|_{\mathrm{M}}=\|\beta\|_{\mathrm{M}}=s_{\mathrm{M}}+8$.

(iii) $s_{\mathrm{M}}=8=\|\lambda\|_{\mathrm{M}}$ and $\|\alpha\|_{\mathrm{M}}=\|\beta\|_{\mathrm{M}} \in\left\{s_{\mathrm{M}}+6, s_{\mathrm{M}}+8\right\}$.

We'll deal with these cases separately.

In Case (i) note first that the line segment of negative slope $\left[\mu, \frac{3}{5} \beta\right]$ lies in $B_{\mathrm{M}}$ and calculation shows that it intersects the horizontal line through $\frac{3}{5} \alpha$ in the point $\frac{3}{5} \alpha+2 \mu$. On the other hand, the fact that $\alpha$ is not a strict boundary class implies that the edge of $\partial B_{\mathrm{M}}$ containing $\left[\frac{3}{5} \alpha, \frac{3}{5} \beta\right]$ extends through $\frac{3}{5} \alpha$ and ends below and to the left of it. It follows that there is a horizontal line lying just below $\frac{3}{5} \alpha$ which intersects $B_{\mathrm{M}}$ in a segment of length strictly longer than 2, which is impossible. Thus Case (i) leads to a contradiction.

Next consider Case (ii). Recall $\|\gamma\|_{\mathrm{M}},\|\eta\|_{\mathrm{M}} \equiv 0(\bmod 4)$ and since the segments $\left[\frac{5}{9} \alpha, \lambda\right]$ and $\left[\mu, \frac{5}{9} \beta\right]$ lie in $B_{\mathrm{M}}$ and it follows that $\|\gamma\|_{\mathrm{M}}=$ $\|\eta\|_{\mathrm{M}}=12$. The segments $\left[-\mu, \frac{5}{9} \alpha\right]$ and $\left[\mu, \frac{5}{6} \eta\right]$ lie in $B_{M}$ and are parallel, so in fact they lie in $\partial B_{\mathrm{M}}$. Hence $\mu$ is not a vertex of $B_{\mathrm{M}}$. Now $\frac{5}{9} \beta \in$ $B_{\mathrm{M}}$ so there is a vertex $v_{1}$ of $B_{\mathrm{M}}$ whose $\lambda$-coordinate is larger than 1 . Actually $v_{1}$ may be taken to lie in the sector bounded by the half-rays based at the origin and passing through $\lambda$ and $\eta$. Let $\left(m_{1}, n_{1}\right) \in L$ be the strict boundary class associated to $v_{1}$. Since $\beta$ is not a strict boundary class we have $\left|n_{1}\right| \geq 3$. Consider the edge of $B_{\mathrm{M}}$ which contains $\left[-\mu, \frac{5}{9} \alpha\right]$. Since $\alpha$ is not a strict boundary slope this edge passes through $\frac{5}{9} \alpha$ to an edge $v_{2}$ associated to a strict boundary class $\left(m_{2}, n_{2}\right)$ where $\left|n_{2}\right| \geq 2$. An application of Lemma 14.2 reveals that $\left|n_{1}\right|=3,\left|n_{2}\right|=2$ and $B_{\mathrm{M}}$ is a parallelogram. Since $\|\lambda\|_{\mathrm{M}}=s_{\mathrm{M}}$ it is easy to see that $\left(m_{1}, n_{1}\right)=(2,3)$ while $\left(m_{2}, n_{2}\right)=(-2,3)$. The Newton polygon of $A_{\mathrm{M}}$ is a parallelogram with vertices $(0,2),(2,5),(5,3)$ and $(0,3)$. In particular since $(0,3)$ is a vertex of $N_{\mathrm{M}}$, Proposition $10.3(5)$ implies that $i_{*}(\mu)=0$, contrary our previous calculations. Thus Case (ii) leads to a contradiction.

Now consider Case (iii) when $\|\alpha\|_{\mathrm{M}}=\|\beta\|_{\mathrm{M}}=s_{\mathrm{M}}+6$. The line segment $\left[\mu, \frac{4}{7} \beta\right]$ lies in $B_{\mathrm{M}}$, which implies that $\|\eta\|_{\mathrm{M}} \leq \frac{11}{8} s_{\mathrm{M}}=11$. Hence since $\|\eta\|_{\mathrm{M}}$ is divisible by $4,\|\eta\|_{\mathrm{M}}=8=s_{\mathrm{M}}$. The segment $[\mu, \eta]$ is therefore contained in $B_{\mathrm{M}}$, as is $\left[-\mu, \frac{4}{7} \alpha\right]$. But then $B_{\mathrm{M}}$ contains a horizontal line segment of length longer than two, which is impossible.

Finally suppose that $\|\alpha\|_{\mathrm{M}}=\|\beta\|_{\mathrm{M}}=s_{\mathrm{M}}+8$ in Case (iii). The line segment $\left[\frac{1}{2} \alpha, \lambda\right]$ lies in $B_{\mathrm{M}}$, which implies that $\|\gamma\|_{\mathrm{M}}=8$ or 12 . If $\|\gamma\|_{\mathrm{M}}=8$, then as $\|\alpha / 2\|_{\mathrm{M}}=s_{\mathrm{M}}$ and lies on $[-\mu, \gamma]$ we see that 
this segment is contained in $\partial B_{\mathrm{M}}$. Similarly since $\|\lambda\|_{\mathrm{M}}=s_{\mathrm{M}}$ and is contained on $\left[\gamma, \frac{1}{2} \beta\right]$, this segment is contained in $\partial B_{\mathrm{M}}$ as well. Thus $\gamma$ is a vertex of $B_{\mathrm{M}}$. Let $e$ be the edge of $B_{\mathrm{M}}$ which contains $\left[\gamma, \frac{1}{2} \beta\right]$ and $v_{1}$ the endpoint of $e$ other than $\gamma$. Now $v_{1} \neq \frac{1}{2} \beta$ since $\beta$ is not a boundary class associated to a vertex of $B_{\mathrm{M}}$. Further Lemma 14.3 shows that $v_{1} \neq \eta=(1,1)$. Then by Lemma 14.1 , we see that $v_{1}=\left(\frac{2}{3}, 1\right)$. Now $\left[v_{1}, \mu\right]$ is contained in $B_{\mathrm{M}}$ which implies that $\|\eta\|_{\mathrm{M}} \leq \frac{4}{3} s_{\mathrm{M}} \leq 11$. Thus since $\|\eta\|_{\mathrm{M}}$ is divisible by $4,\|\eta\|_{\mathrm{M}}=8$. But then $[\gamma, \eta]$ is contained in $\partial B_{\mathrm{M}}$, which contradicts Lemma 14.3. Therefore we have $\|\gamma\|_{\mathrm{M}}=12$.

The segment $\left[\mu, \frac{1}{2} \beta\right]$ lies in $B_{\mathrm{M}}$ and so $\|\eta\|_{\mathrm{M}}=8$ or 12 . In the former case the parallel segments $[\mu, \eta],[-\mu, \alpha / 2]$ lie in $B_{\mathrm{M}}$ and have horizontal separation equal to 2 , so in particular they lie in $\partial B_{\mathrm{M}}$ and therefore $\mu$ is not a vertex of $B_{\mathrm{M}}$. Further we have $[\eta, \lambda] \subset \partial B_{\mathrm{M}}$ as $\|\beta / 2\|_{\mathrm{M}}=s_{\mathrm{M}}$. Thus $\eta$ is a vertex of $B_{\mathrm{M}}$. By Lemma $14.3, \gamma$ cannot be a vertex of $B_{\mathrm{M}}$, and so since $\alpha$ is not a strict boundary class, Lemma 14.1 can be used to prove that $B_{\mathrm{M}}$ is the polygon with vertices $\pm \eta, \pm \lambda$ and $\pm(-1,2 / 3)$. But then $N_{\mathrm{M}}$ has a vertex at $(3,0)$, which implies that $i_{*}(\mu)=0$ (Proposition $10.3(5)$ ), contrary to our previous calculations. Thus $\|\eta\|_{\mathrm{M}}=12$. It follows that $\left[\mu, \frac{1}{2} \beta\right] \subset \partial B_{\mathrm{M}}$ and since $\beta$ is not a strict boundary class, this segment extends upward along an edge of $B_{\mathrm{M}}$ to a vertex, say $v_{1}$, of $B_{\mathrm{M}}$, whose $\lambda$-coordinate is larger than 1 . If $v_{1}$ is a positive multiple of a primitive class $(m, n) \in L$, then Lemma 14.1 implies that that $n=3$ and since $\lambda \in \partial B_{\mathrm{M}}$, an easy calculation shows that $v_{1}$ must be the point $\left(\frac{2}{5}, \frac{6}{5}\right)$, which is the intersection of the two edges of $B_{\mathrm{M}}$ containing the segments $\left[\mu, \frac{1}{2} \beta\right]$ and $\left[\frac{1}{2} \alpha, \lambda\right]$. Lemma 14.1 now shows that $B_{\mathrm{M}}$ has at most one other vertex pair different from $\pm \mu$, and this pair corresponds to an integral class. Owing to the fact that $\alpha$ is not a strict boundary class, we deduce that $B_{\mathrm{M}}$ must be the parallelogram with vertices $\pm\left(\frac{2}{5}, \frac{6}{5}\right)$ and $\pm\left(-\frac{6}{5}, \frac{2}{5}\right)$. But then $N_{\mathrm{M}}$ is the parallelogram with vertices $(3,0),(0,1),(1,4)$ and $(4,3)$. In particular Proposition $10.3(5)$ implies that $i_{*}(\mu)=0$, contrary to our previous calculations. This final contradiction completes the proof of the lemma.

q.e.d.

According to the three previous lemmas, $\alpha \notin \mathcal{F}_{0}$. This contravenes our hypotheses and so completes the proof of Proposition 12.5. q.e.d.

\section{Proof of Proposition 12.6}

If there is no half-integral class in $\mathcal{F}_{0}$, then Proposition 12.6 holds 
by Propositions 12.3 and 12.4. So we may assume that there is some $\beta \in \mathcal{F}_{0}$ whose $\lambda$-coordinate is 2 . Without loss of generality, we may assume that $\beta=(1,2)$. Applying Propositions 12.3 and 12.5 , we see that $\mathcal{F}_{0} \subset\{\beta, \alpha=(-1,1), \lambda=(0,1), \eta=(1,1), \gamma=(2,1), m=(1,0)\}$. If Proposition 12.6 is not true, then $\alpha, \gamma \in \mathcal{F}_{0}$. We shall assume this in order to derive a contradiction.

We start by developing some useful inequalities.

\section{Lemma 17.1.}

(1) $s_{M}<\|\alpha\|_{M},\|\beta\|_{M},\|\gamma\|_{M}$.

(2) $\|\lambda\|_{M},\|\eta\|_{M}<\|\beta\|_{M}$.

(3) (i) $3\|\lambda\|_{M} \leq\|\alpha\|_{M}+\|\beta\|_{M}$ with equality if and only if $\left[\frac{s_{M}}{\|\alpha\|_{M}} \alpha, \frac{s_{M}}{\|\beta\|_{M}} \beta\right] \subset \partial B_{M}$.

(ii) If $3 s_{M}=\|\alpha\|_{M}+\|\beta\|_{M}$, then $\lambda \in \partial B_{M}$.

(4) (i) $3\|\lambda\|_{M} \leq\|\gamma\|_{M}+\|\beta\|_{M}$ with equality if and only if $\left[\frac{s_{M}}{\|\gamma\|_{M}} \gamma, \frac{s_{M}}{\|\beta\|_{M}} \beta\right] \subset \partial B_{M}$.

(ii) If $3 s_{M}=\|\gamma\|_{M}+\|\beta\|_{M}$, then $\lambda \in \partial B_{M}$.

Proof. (1) The inequality $\|\beta\|_{\mathrm{M}}>s_{\mathrm{M}}$ follows from Proposition 4.5. Suppose next that $\|\alpha\|_{\mathrm{M}}=s_{\mathrm{M}}$. Then $\|\beta\| \geq 2 s_{\mathrm{M}}$ as otherwise $\lambda$ would be contained in the interior of $B_{\mathrm{M}}$. If $\|\beta\|=2 s_{\mathrm{M}}$ then as neither $\alpha$ nor $\beta$ are strict boundary classes, $[\alpha, \beta / 2]$ is contained in the interior of an edge of $\partial B_{\mathrm{M}}$. Let $v$ be the endpoint of this edge which has negative $\mu$-coordinate. Then $[-\mu, v] \subset B_{\mathrm{M}}$. Hence if $\|\gamma\|_{\mathrm{M}} \leq 2 s_{\mathrm{M}}$ then the line $\{(x, 1 / 2) \mid x \in \mathbb{R}\}$ intersects $B_{\mathrm{M}}$ in a segment of length larger than 2 . As this is impossible it follows that either $\|\beta\|_{\mathrm{M}}>2 s_{\mathrm{M}}$ or $\|\gamma\|_{\mathrm{M}}>2 s_{\mathrm{M}}$. If $\|\gamma\|_{\mathrm{M}}>2 s_{\mathrm{M}}$, then $\gamma$ is of type $O(2)$ or $O(3)$ by Proposition 9.3. In the former case we have $2 s_{\mathrm{M}}<\|\gamma\|_{\mathrm{M}} \leq\|\lambda\|_{\mathrm{M}}=\|\alpha+\mu\|_{\mathrm{M}} \leq 2 s_{\mathrm{M}}$, which is obviously impossible. The latter is ruled out by the inequalities $2 s_{\mathrm{M}}<\|\gamma\|_{\mathrm{M}} \leq\|\alpha\|_{\mathrm{M}}=s_{\mathrm{M}}$. A similar argument shows that $\|\beta\|_{\mathrm{M}}$ cannot be larger than $2 s_{\mathrm{M}}$. Hence $s_{\mathrm{M}}<\|\alpha\|_{\mathrm{M}}$ and an identical argument gives $s_{\mathrm{M}}<\|\gamma\|_{\mathrm{M}}$.

(2) This may be deduced in the same manner that was used to prove Lemma 16.1 (1).

(3) (i) The line segment $\left[\frac{s_{\mathrm{M}}}{\|\alpha\|_{\mathrm{M}}} \alpha, \frac{s_{\mathrm{M}}}{\|\beta\|_{\mathrm{M}}} \beta\right]$ lies entirely in $B_{\mathrm{M}}$ and intersects the $\lambda$-axis at $\left(0, \frac{3 s_{\mathrm{M}}}{\|\alpha\|_{\mathrm{M}}+\|\beta\|_{\mathrm{M}}}\right)$. On the other hand $\partial B_{\mathrm{M}}$ intersects the positive $\lambda$-axis at $\left(0, \frac{s_{\mathrm{M}}}{\|\lambda\|_{\mathrm{M}}}\right)$ and so $\frac{3 s_{\mathrm{M}}}{\|\alpha\|_{\mathrm{M}}+\|\beta\|_{\mathrm{M}}} \leq \frac{s_{\mathrm{M}}}{\|\lambda\|_{\mathrm{M}}}$, 
i.e., $3\|\lambda\|_{\mathrm{M}} \leq\|\alpha\|_{\mathrm{M}}+\|\beta\|_{\mathrm{M}}$. Further if there is equality then the three points $\frac{s_{\mathrm{M}}}{\|\alpha\|_{\mathrm{M}}} \alpha, \frac{s_{\mathrm{M}}}{\|\lambda\|_{\mathrm{M}}} \lambda, \frac{s_{\mathrm{M}}}{\|\beta\|_{\mathrm{M}}} \beta$ of norm $s_{\mathrm{M}}$ all lie on $\left[\frac{s_{\mathrm{M}}}{\|\alpha\|_{\mathrm{M}}} \alpha, \frac{s_{\mathrm{M}}}{\|\beta\|_{\mathrm{M}}} \beta\right]$. Thus $\left[\frac{s_{\mathrm{M}}}{\|\alpha\|_{\mathrm{M}}} \alpha, \frac{s_{\mathrm{M}}}{\|\beta\|_{\mathrm{M}}} \beta\right] \subset \partial B_{\mathrm{M}}$.

(ii) If $3 s_{\mathrm{M}}=\|\alpha\|_{\mathrm{M}}+\|\beta\|_{\mathrm{M}}$, then part (i) shows that $s_{\mathrm{M}}=\|\lambda\|_{\mathrm{M}}$.

(4) This follows as in part (3).

q.e.d.

The following lemma is a consequence of Proposition 9.3 and Lemma 17.1.

Lemma 17.2. $\beta$ cannot have type $C, D, Q$ or $T(q), O(q), I(q)$ where $q \leq 2$.

Lemma 17.3. $\beta$ cannot have type $O(3)$.

Proof. If $\beta$ is of type $O(3)$ then so are $\alpha$ and $\gamma$ (Proposition 3.3). Theorem 1.5 of $[2]$ implies that $H_{1}\left(M ; \mathbb{Z}_{2}\right)=\mathbb{Z}_{2} \oplus \mathbb{Z}_{2}$ and so from Lemma 2.1 we deduce that $i_{*}(\mu)=i_{*}(\beta) \neq 0$. Thus either $i_{*}(\gamma)=0$ or $i_{*}(\alpha)=0$. But then $H_{1}\left(M(\alpha) ; \mathbb{Z}_{2}\right)=\mathbb{Z}_{2} \oplus \mathbb{Z}_{2}$ or $H_{1}\left(M(\gamma) ; \mathbb{Z}_{2}\right)=$ $\mathbb{Z}_{2} \oplus \mathbb{Z}_{2}$, contrary to Lemma 2.1 . Thus $\beta$ is not of type $O(3)$. q.e.d.

Lemma 17.4. $\beta$ cannot have type $T(3)$ or $I(3)$.

Proof. If $\beta$ is of type $T(3)$ or $I(3)$, then both $\alpha$ and $\gamma$ have the same type as $\beta$. In particular $H_{1}\left(M(\beta) ; \mathbb{Z}_{2}\right)=H_{1}\left(M(\alpha) ; \mathbb{Z}_{2}\right)=H_{1}\left(M(\gamma) ; \mathbb{Z}_{2}\right)$ $=0$ and $H_{1}\left(M ; \mathbb{Z}_{2}\right)=\mathbb{Z}_{2}$. Thus $i_{*}(\mu)=i_{*}(\beta) \neq 0$ but either $i_{*}(\gamma)=0$ or $i_{*}(\alpha)=0$. Then $H_{1}\left(M(\alpha) ; \mathbb{Z}_{2}\right)=\mathbb{Z}_{2}$ or $H_{1}\left(M(\gamma) ; \mathbb{Z}_{2}\right)=\mathbb{Z}_{2}$, giving a contradiction. Thus $\beta$ cannot have type $T(3)$ or $I(3)$. $\quad$ q.e.d.

Lemma 17.5. $\beta$ cannot have type $O(4)$.

Proof. Suppose otherwise. Then Proposition 10.3 implies that $H_{1}\left(M ; \mathbb{Z}_{2}\right)=\mathbb{Z}_{2}$, and thus $i_{*}(\mu)=i_{*}(\beta)=0$. Hence $i_{*}(\alpha)=i_{*}(\gamma) \neq 0$. Therefore by Lemma 17.1 (1), both $\alpha$ and $\gamma$ have type $I$ or $T$. Also Proposition 9.6 implies that $\|\mu\|_{\mathrm{M}}$ and $\|\beta\|_{\mathrm{M}}$ are divisible by 4 . Hence as $s_{\mathrm{M}}<\|\beta\|_{\mathrm{M}} \leq s_{\mathrm{M}}+6$ we deduce that $\|\beta\|_{\mathrm{M}}=s_{\mathrm{M}}+4$. Now $3 s_{\mathrm{M}} \leq$ $\|\alpha\|+\|\beta\| \leq 2 s_{\mathrm{M}}+14$ (recall $\alpha$ has type $T$ or $I$ ) and so $s_{\mathrm{M}} \leq 14$. But $s_{\mathrm{M}}=\|\mu\|_{\mathrm{M}}$ is divisible by 4 , so $s_{\mathrm{M}}=8$ or 12 . In the latter eventuality Lemma 17.1 (3), (4) implies that:

- $\|\alpha\|_{\mathrm{M}}=20,\|\lambda\|_{\mathrm{M}}=12$ and $\left[\frac{3}{5} \alpha, \frac{3}{4} \beta\right] \subset \partial B_{\mathrm{M}}$.

- $\|\gamma\|_{\mathrm{M}}=20,\|\eta\|_{\mathrm{M}}=12$ and $\left[\frac{3}{5} \gamma, \frac{3}{4} \beta\right] \subset \partial B_{\mathrm{M}}$. 
Therefore $\frac{3}{4} \beta$ is a vertex of $B_{\mathrm{M}}$, contrary to our assumptions, and so $s_{\mathrm{M}}=8$. Another application of Lemma 17.1 (3), (4) shows that $\|\alpha\|_{\mathrm{M}},\|\gamma\|_{\mathrm{M}} \geq 12$ while $\|\lambda\|_{\mathrm{M}}=\|\eta\|_{\mathrm{M}}=8$.

Since the point $\frac{2}{3} \beta$ is in $B_{\mathrm{M}}$, the polygon $B_{\mathrm{M}}$ has a vertex whose associated boundary class has $\lambda$-coordinate at least three. According to Lemma 14.1 there are only four possible shapes for $B_{\mathrm{M}}$ :

(i) a parallelogram with vertices $\pm \frac{1}{2}(\mu+3 \lambda)$ and $\pm \frac{1}{2}(3 \mu+\lambda)$,

(ii) a parallelogram with vertices $\pm \frac{1}{2}(-2 \mu+\lambda)$ and $\pm \frac{1}{2}(2 \mu+3 \lambda)$,

(iii) a polygon with vertices $\mu, \pm \frac{1}{2}(\mu+3 \lambda)$ and $\eta$,

(iv) a polygon with vertices $\mu, \lambda$ and $\pm \frac{1}{2}(2 \mu+3 \lambda)$.

In each of these four cases one verifies that the associated Newton polygon has a corner at a lattice point whose second coordinate is odd. But we have already shown that $i_{*}(\mu)=0$ while $i_{*}(\lambda) \neq 0$ and so Proposition $10.3(5)$ shows that $A_{\mathrm{M}}(u, v)$ involves only even powers of $v$. This contradiction completes the proof that $\beta$ cannot be of type $O(4)$. q.e.d.

Lemma 17.6. $\beta$ cannot have type $I(5)$.

Proof. Suppose otherwise. Then $H_{1}\left(M ; \mathbb{Z}_{2}\right)=\mathbb{Z}_{2}$ and $i_{*}(\mu)=$ $i_{*}(\beta) \neq 0$. Thus exactly one of $\alpha$ and $\gamma$ is not in $\operatorname{ker}\left(H_{1}\left(\partial M ; \mathbb{Z}_{2}\right) \rightarrow\right.$ $\left.H_{1}\left(M ; \mathbb{Z}_{2}\right)\right)$. We will treat the case $\alpha \notin \operatorname{ker}\left(H_{1}\left(\partial M ; \mathbb{Z}_{2}\right) \rightarrow H_{1}\left(M ; \mathbb{Z}_{2}\right)\right)$, the other can be handled similarly.

Now our hypotheses imply that $H_{1}\left(M(\alpha) ; \mathbb{Z}_{2}\right)=0$ while $H_{1}\left(M(\gamma) ; \mathbb{Z}_{2}\right)=\mathbb{Z}_{2}$. Thus $\alpha$ is either a $T$ or $I$-type class and $\gamma$ is either a $D$ or $O$-type class (neither $\alpha$ nor $\gamma$ has $C$-type by Lemma 17.1 (1)). We also have

$$
\|\lambda\|_{\mathrm{M}} \equiv\|\gamma\|_{\mathrm{M}} \equiv 0(\bmod 4)
$$

by Proposition 9.6.

First note that $\gamma$ cannot be of type $O(3)$ because $\beta$ is of $I$-type and $\Delta(\beta, \gamma)=3$. Next assume that $\gamma$ is of type $O(2)$ or $D$ so that $\|\gamma\|_{\mathrm{M}} \leq\|\lambda\|_{\mathrm{M}}$. Then by Lemma 17.1 (1) we have $\|\lambda\|_{\mathrm{M}}>s_{\mathrm{M}}$ and so part (3)(i) of that result implies $3\left(s_{\mathrm{M}}+2\right) \leq 2 s_{\mathrm{M}}+16$, or $s_{\mathrm{M}} \leq 10$. If $s_{\mathrm{M}}=10$, then Lemma 17.1 gives $10<\|\lambda\|_{\mathrm{M}} \leq 12$ and so $\|\lambda\|_{\mathrm{M}}=12$. We also have $\|\gamma\|_{\mathrm{M}}=12$ since $10<\|\gamma\|_{\mathrm{M}} \leq\|\lambda\|_{\mathrm{M}}$ and $\|\gamma\|_{\mathrm{M}}$ is divisible by 4. Lemma 17.1 now implies that $\|\beta\|_{\mathrm{M}}=\|\alpha\|_{\mathrm{M}}=s_{\mathrm{M}}+8$ and $\|\eta\|_{\mathrm{M}}=$ 10. Hence the line segments $\left[\frac{s_{\mathrm{M}}}{\|\alpha\|_{\mathrm{M}}} \alpha, \frac{s_{\mathrm{M}}}{\|\beta\|_{\mathrm{M}}} \beta\right]$ and $\left[\frac{s_{\mathrm{M}}}{\|\gamma\|_{\mathrm{M}}} \gamma, \frac{s_{\mathrm{M}}}{\|\beta\|_{\mathrm{M}}} \beta\right]$ are 
contained in $\partial B_{\mathrm{M}}$. But this is impossible as it implies $\frac{s_{\mathrm{M}}}{\|\beta\|_{\mathrm{M}}} \beta$ is a vertex of $B_{\mathrm{M}}$. Hence $s_{\mathrm{M}} \neq 10$. If $s_{\mathrm{M}}=8$, Lemma 17.1 implies that $\|\lambda\|_{\mathrm{M}}=8=$ $s_{\mathrm{M}}$ (recall it is divisible by 4 ), contradicting the fact that $\|\lambda\|_{\mathrm{M}}>s_{\mathrm{M}}$. Thus $\gamma$ cannot be of type $D$ or $O(2)$.

Finally suppose that $\gamma$ is of type $O(4)$. Then $\|\gamma\|_{\mathrm{M}} \leq s_{\mathrm{M}}+6$ and so by Lemma 17.1 we see that $s_{\mathrm{M}} \leq 14$.

If $s_{\mathrm{M}}=14$, then by Lemma 17.1 we obtain $\|\lambda\|_{\mathrm{M}}=14$, which is not divisible by 4 , a contradiction.

Next suppose that $s_{\mathrm{M}}=12$. Then Lemma 17.1 implies that $\|\lambda\|_{\mathrm{M}}=$ $\|\eta\|_{\mathrm{M}}=12$. As $\|\gamma\|_{\mathrm{M}}>s_{\mathrm{M}}$ and divisible by $4,\|\gamma\|_{\mathrm{M}}=16$. Hence from Lemma 17.1 we see that $\|\beta\|_{\mathrm{M}}=20$ and $\left[\frac{s_{\mathrm{M}}}{\|\gamma\|_{\mathrm{M}}} \gamma, \frac{s_{\mathrm{M}}}{\|\beta\|_{\mathrm{M}}} \beta\right] \subset \partial B_{\mathrm{M}}$. Now $\frac{s_{\mathrm{M}}}{\|\gamma\|_{\mathrm{M}}} \gamma$ is not a vertex of $B_{\mathrm{M}}$ and so if $v \in\left(\mu, \frac{s_{\mathrm{M}}}{\|\gamma\|_{\mathrm{M}}} \gamma\right)$ we have $\|v\|_{\mathrm{M}}<s_{\mathrm{M}}$. Hence for such $v$ we have $\|v-2 \mu\|_{\mathrm{M}}>s_{\mathrm{M}}$. In particular $\frac{3}{5} \alpha+2 \mu \in\left(\mu, \frac{s_{\mathrm{M}}}{\|\gamma\|_{\mathrm{M}}} \gamma\right)$ so $\left\|\frac{3}{5} \alpha\right\|_{\mathrm{M}}>s_{\mathrm{M}}=12$, or $\|\alpha\|_{\mathrm{M}}>20=s_{\mathrm{M}}+8$, a contradiction. Therefore $s_{\mathrm{M}} \neq 12$.

Now consider the case where $s_{\mathrm{M}}=10$. By Lemma 17.1 we have $\|\eta\|_{\mathrm{M}}=10$ and $\|\lambda\|_{\mathrm{M}}=12$ (note again that $\|\lambda\|_{\mathrm{M}}$ divisible by 4 ). Hence Lemma 17.1 implies that $\|\alpha\|_{\mathrm{M}}=\|\beta\|_{\mathrm{M}}=18$. Further the segment $\left[\frac{s_{\mathrm{M}}}{\|\alpha\|_{\mathrm{M}}} \alpha, \frac{s_{\mathrm{M}}}{\|\beta\|_{\mathrm{M}}} \beta\right] \subset \partial B_{\mathrm{M}}$. Lemma 17.1 also gives $12 \leq\|\gamma\|_{\mathrm{M}}$. Hence $\|\gamma\|_{M}=12$ or 16 . In fact $\|\gamma\|_{M}=16$. To see this suppose that it is 12. Then $3\|\eta\|_{\mathrm{M}}=\|\gamma\|_{\mathrm{M}}+\|\beta\|_{\mathrm{M}}$ and therefore the segment $\left[\frac{s_{\mathrm{M}}}{\|\gamma\|_{\mathrm{M}}} \gamma, \frac{s_{\mathrm{M}}}{\|\beta\|_{\mathrm{M}}} \beta\right] \subset \partial B_{\mathrm{M}}$. But then $\frac{s_{\mathrm{M}}}{\|\beta\|_{\mathrm{M}}} \beta=\left[\frac{s_{\mathrm{M}}}{\|\alpha\|_{\mathrm{M}}} \alpha, \frac{s_{\mathrm{M}}}{\|\beta\|_{\mathrm{M}}} \beta\right] \cap$ $\left[\frac{s_{\mathrm{M}}}{\|\gamma\|_{\mathrm{M}}} \gamma, \frac{s_{\mathrm{M}}}{\|\beta\|_{\mathrm{M}}} \beta\right]$ is a vertex of $B_{\mathrm{M}}$, implying that $\beta$ is a strict boundary class, a fact our hypotheses exclude. Thus $\|\gamma\|_{\mathrm{M}}=16$. Next observe that since $s_{\mathrm{M}}=10$ and $\beta$ is not a strict boundary class, the segment $\left[\frac{s_{\mathrm{M}}}{\|\alpha\|_{\mathrm{M}}} \alpha, \frac{s_{\mathrm{M}}}{\|\beta\|_{\mathrm{M}}} \beta\right] \subset \partial B_{\mathrm{M}}$ extends past $\frac{s_{\mathrm{M}}}{\|\beta\|_{\mathrm{M}}} \beta$ to a vertex $v_{1}$ of $B_{\mathrm{M}}$ corresponding to a boundary class $m \mu+n \lambda$ where $3 \leq n \leq 5$ and $1 \leq \frac{n}{m}<2$ (Lemma 14.2). We claim $n \neq 5$. For otherwise $B_{\mathrm{M}}$ would be a parallelogram with vertices $\pm \mu$ and $\pm v_{1}$ which is impossible as $\|\gamma\|_{\mathrm{M}}<2 s_{\mathrm{M}}$. So $(m, n)=(2,3)$ or $(3,4)$. The latter case can be excluded as it would imply that $\eta$ lies in the interior of $B_{\mathrm{M}}$. Thus $v_{1}$ lies on the line of slope $\frac{3}{2}$ through the origin. One can now verify that $\left[v_{1}, \frac{s_{\mathrm{M}}}{\|\gamma\|_{\mathrm{M}}} \gamma\right] \subset \partial B_{\mathrm{M}}$. Since $\operatorname{both}\left[\frac{s_{\mathrm{M}}}{\|\alpha\|_{\mathrm{M}}} \alpha, \frac{s_{\mathrm{M}}}{\|\beta\|_{\mathrm{M}}} \beta\right]$ and $\left[v_{1}, \frac{s_{\mathrm{M}}}{\|\gamma\|_{\mathrm{M}}} \gamma\right]$ lie in $\partial B_{\mathrm{M}}$, they extend downwards to two more vertices $v_{2}$ and $v_{3}$ of $B_{\mathrm{M}}$ whose associated boundary classes must be integral (Lemma 14.2). By hypothesis $\alpha$ and $\gamma$ are not strict boundary classes, so $v_{2}$ lies on a line through the origin of slope 
$-\frac{1}{k}$ where $k$ is an integer which is at least 2 , and $v_{2}$ lies on the line of slope $\frac{1}{j}$ through the origin where $j$ is at least 3 . But this is impossible as one can now easily see that $B_{\mathrm{M}}$ contains a horizontal segment of length longer than 2. This contradiction completes the proof that $s_{\mathrm{M}} \neq 10$.

Finally assume that $s_{\mathrm{M}}=8$. Since $\|\lambda\|_{\mathrm{M}}$ is divisible by 4 and $3\|\lambda\|_{\mathrm{M}} \leq\|\alpha\|_{\mathrm{M}}+\|\beta\|_{\mathrm{M}} \leq 2 s_{\mathrm{M}}+16=32$, we have $\|\lambda\|_{\mathrm{M}}=8$. Also $3\|\eta\|_{\mathrm{M}} \leq\|\gamma\|_{\mathrm{M}}+\|\beta\|_{\mathrm{M}} \leq 2 s_{\mathrm{M}}+14=30$ and therefore $\|\eta\|_{\mathrm{M}}=8$ or 10. Now $s_{\mathrm{M}}<\|\gamma\|_{\mathrm{M}} \leq s_{\mathrm{M}}+6=14$ and $\|\gamma\|_{\mathrm{M}}$ is divisible by 4 , so in fact $\|\gamma\|_{\mathrm{M}}=12$. If we now assume that $\|\eta\|_{\mathrm{M}}=10$, then we obtain the impossible relation $30=3\|\eta\|_{\mathrm{M}} \leq\|\gamma\|_{\mathrm{M}}+\|\beta\|_{\mathrm{M}} \leq 12+16=28$. Hence $\|\eta\|_{\mathrm{M}}=8$. Note that $\|\beta\|_{\mathrm{M}} \leq s_{\mathrm{M}}+8=16$. We shall consider two cases $\|\beta\|_{\mathrm{M}}<16$ and $\|\beta\|_{\mathrm{M}}=16$ separately.

Suppose first of all that $\|\beta\|_{\mathrm{M}}<16=2 s_{\mathrm{M}}$ and let $v_{1}=u \mu+v \lambda$ be the vertex of $B_{\mathrm{M}}$ which has the maximal $\lambda$-coordinate. Let $m \mu+n \lambda$ be a primitive class which is a positive rational multiple of $v_{1}$. Since $\|\beta\|_{\mathrm{M}}<2 s_{\mathrm{M}}$ and $\beta$ is not a strict boundary class, $n>2$. Now by Lemma $14.1, v_{1}$ is the unique vertex of $B_{\mathrm{M}}$ whose $\lambda$-coordinate is larger than 1 . It follows that $\left[v_{1}, \lambda\right]$ and $\left[v_{1}, \eta\right]$ are contained in $\partial B_{\mathrm{M}}$ and thus extend downward to two more vertices of $B_{\mathrm{M}}$ which can be easily seen to be distinct from $\pm \mu$. But this contradicts Lemma 14.1.

Finally suppose that $\|\beta\|_{\mathrm{M}}=16$. Then $\|\lambda\|_{\mathrm{M}}=\|\beta / 2\|_{\mathrm{M}}=\|\eta\|_{\mathrm{M}}=$ $8=s_{\mathrm{M}}$. It follows that the segment $[\lambda, \eta] \subset \partial B_{\mathrm{M}}$. If $\eta$ is not a vertex of $B_{\mathrm{M}}$, then $B_{\mathrm{M}}$ is a parallelogram by Proposition $9.6(2)$ and the segment $[\lambda, \eta]$ extends to a vertex $v_{1}$ which is either $\frac{4}{3} \mu+\lambda, \frac{3}{2} \mu+\lambda$ or $\frac{5}{3} \mu+$ $\lambda$. The first and third are ruled out by the fact that if either were a vertex then $\lambda$ would be as well (Lemma 14.1). But then $B_{\mathrm{M}}$ would not be a parallelogram. Thus $v_{1}=\frac{3}{2} \mu+\lambda$. We see then that $B_{\mathrm{M}}$ is the parallelogram with vertices $\pm\left(-\frac{1}{2}, 1\right)$ and $\pm\left(\frac{3}{2}, 1\right)$. So the Newton polygon $N_{\mathrm{M}}$ is the parallelogram with vertices $(1,0),(0,2),(3,4)$ and $(4,2)$. Hence the $A$-polynomial $A_{\mathrm{M}}(u, v)$ contains $u$ (up to sign) as a monomial, which contradicts to Proposition 10.3 (5).

Assume now that $\eta$ is a vertex of $B_{\mathrm{M}}$. By Proposition $9.6(1), B_{\mathrm{M}}$ contains at most two more vertex pairs. Since $\frac{2}{3} \gamma=\left(\frac{4}{3}, \frac{2}{3}\right)$ is in $\partial B_{\mathrm{M}}$, there is vertex $v_{1}$ of $B_{\mathrm{M}}$ whose $\mu$-coordinate is larger than 1 . Since $\alpha$ is not a vertex of $B_{\mathrm{M}}$, one can easily see that $B_{\mathrm{M}}$ has two vertices with nonpositive $\mu$-coordinates and positive $\lambda$-coordinates. So $B_{\mathrm{M}}$ would have at least four pairs of vertices, which contradicts Lemma 14.1. Therefore $\gamma$ cannot be of type $O(4)$.

q.e.d.

The above lemmas imply that $\beta \notin \mathcal{F}_{0}$. This contradiction completes 
the proof of Proposition 12.6.

q.e.d.

\section{Finite surgery on small knots in $S^{3}$}

In this section we make some general remarks concerning finite surgery on small knots in the 3 -sphere, i.e., those knots $K$ whose exteriors $M_{K}$ contain no closed essential surface, and show how the theory developed previously in this paper can be used to give a quick proof of the classification of the finite surgery slopes of 2-bridge knots (due to Delman [14] and independently to Tanguay [32]).

The finite surgery slopes on $\partial M_{K}$ have been classified when $K$ is a torus knot [27] and a satellite knot [2], so we only need to consider small hyperbolic knots. Fix such a knot and let $M$ denote its exterior, $C_{\mathrm{M}}$ the canonical norm curve in $X(M)$, and $B_{\mathrm{M}}$ the canonical norm polygon. It is a consequence of Theorem 2.0.3 of [11] that the finite filling slopes on $\partial M$ are not boundary slopes. Hence the canonical norm of a finite filling class is subject to the constraints imposed by Proposition 9.3 and so in particular if $\{\mu, \lambda\}$ denotes the standard meridian-longitude basis of $H_{1}(\partial M),\|\mu\|_{\mathrm{M}}=s_{\mathrm{M}}$ and $\mu$ is not a vertex of $B_{\mathrm{M}}$. Consider a nonmeridinal finite filling class on $\partial M$. According to Proposition 9.3 we have $\|\alpha\|_{\mathrm{M}} \leq \max \left\{2 s_{\mathrm{M}}, s_{\mathrm{M}}+8\right\}$. Therefore

$$
\begin{cases}\alpha \in 3 B_{\mathrm{M}} & \text { if } s_{\mathrm{M}}=4 \\ \alpha \in \frac{7}{3} B_{\mathrm{M}} & \text { if } s_{\mathrm{M}}=6 \\ \alpha \in 2 B_{\mathrm{M}} & \text { if } s_{\mathrm{M}} \geq 8 .\end{cases}
$$

We also know that the absolute value of the $\lambda$-coordinate of $\alpha$ is strictly less than than 2 (Theorem 1.2). Set

$$
h\left(B_{\mathrm{M}}\right)=\sup \left\{y \mid \text { there is an } x \text { such that } x \mu+y \lambda \in B_{\mathrm{M}}\right\} .
$$

Proposition 18.1. Suppose that $K \subset S^{3}$ is a small hyperbolic knot with exterior $M$, and that $\alpha$ is a non-meridinal finite filling class on $\partial M$.

(1) The inequality $h\left(B_{M}\right) \geq \frac{1}{2}$ holds.

(2) If $\alpha=m \mu+2 \lambda$ for some odd integer $m$, then $\alpha$ is of type $T(3), I(3)$ or $I(5)$. Further $s_{M} \geq 8$ and $h\left(B_{M}\right) \geq 1$.

Proof. (1) We observed above that if $s_{\mathrm{M}} \geq 8$ then $\alpha \in 2 B_{\mathrm{M}}$, and so $h\left(B_{\mathrm{M}}\right) \geq \frac{1}{2}$. If $s_{\mathrm{M}}=4$, then $\alpha \in 3 B_{\mathrm{M}}$ so that $h\left(B_{\mathrm{M}}\right) \geq \frac{1}{3}$. But since $\mu$ is 
not a vertex, Subcase I. 1 of $\S 13$ implies that there is an integer $n \geq 1$ such that $h\left(B_{\mathrm{M}}\right)=\frac{1}{2 n}$. Hence we must have $h\left(B_{\mathrm{M}}\right)=\frac{1}{2}$.

Finally assume that $s_{\mathrm{M}}=6$. Then $\alpha \in \frac{7}{3} B_{\mathrm{M}}$ and so $h\left(B_{\mathrm{M}}\right) \geq \frac{3}{7}$. According to Subcase II.1 of $\S 13$, either $h\left(B_{\mathrm{M}}\right)=\frac{3}{k}$ for some integer $k \geq 3$ (Subcase II.1.b), or $h\left(B_{\mathrm{M}}\right)=\frac{3}{j+q}$ for some even integers $j, q \geq 2$ (Subcase II.1.c). In the latter case the inequality $h\left(B_{\mathrm{M}}\right) \geq \frac{3}{7}$ implies that $j+q \in\{4,6\}$ and therefore $h\left(B_{\mathrm{M}}\right) \geq \frac{1}{2}$. In the former we see that $k \in\{3,4,5,6,7\}$. But if $k \leq 6$, then $h\left(B_{\mathrm{M}}\right) \geq \frac{1}{2}$, while the case $k=7$ cannot arise because otherwise it follows from Subcase II.1.b of $\S 13$ that the only non-meridinal primitive class in $\frac{7}{3} B_{\mathrm{M}}$ is a vertex of $B_{\mathrm{M}}$, and so $\alpha$ would be a boundary class.

(2) If $\alpha=m \mu+2 \lambda$ is a finite filling class of $K$, the cyclic surgery theorem [11] implies that it is not of $C$-type. Thus since $m$ is odd, it must be either $T$-type or $I$-type (see $\S 2$ ). Since $\alpha$ is not a boundary class, $\alpha \notin \partial B_{\mathrm{M}}$ (Proposition 4.5), and therefore $\|\alpha\|_{\mathrm{M}}>s_{\mathrm{M}}$. It follows that $\alpha$ has type $T(3), I(3)$ or $I(5)$ (Proposition 9.3).

We observed above that if $s_{\mathrm{M}} \geq 8$, then $\alpha \in 2 B_{\mathrm{M}}$, and so $h\left(B_{\mathrm{M}}\right) \geq 1$. To complete the proof we note that under our hypotheses, $s_{\mathrm{M}} \neq 4$ or 6. For instance if $s_{\mathrm{M}}=4$, then $\alpha \in 3 B_{\mathrm{M}}$ so that $h\left(B_{\mathrm{M}}\right) \geq \frac{2}{3}$. But this contradicts Subcase II. 1 of $\S 13$ which implies that $h\left(B_{\mathrm{M}}\right) \leq \frac{1}{2}$. Finally if $s_{\mathrm{M}}=6$, consideration of Subcase II.1 of $\S 13$ shows that the only possibility is for $B_{M}$ to be a parallelogram with vertices $\pm(m, 1)$ and $\pm((m+k) / 2,1 / 2)$, for some integer $m$ and $\alpha$ to be $(2 m+1) \mu+2 \lambda$. In this case $\|\alpha\|_{\mathrm{M}}=14=s_{\mathrm{M}}+8$ and so $\alpha$ has type $I$. But this cannot be as the shape of $B_{\mathrm{M}}$ implies that $\alpha$ does not have type $I(q)$ for any $q \in\{3,5\}$ (Proposition 9.3). Hence the case $s_{\mathrm{M}}=6$ does not arise either.

q.e.d.

The 2-bridge knots are an interesting collection of knots in the 3sphere (see, for instance, [7]). According to [18], they are small knots and further their boundary classes are even integer classes, i.e., they have the form $2 p \mu+\lambda$ for some integer $p$.

Theorem 18.2. A hyperbolic 2-bridge knot admits no nontrivial finite surgery slope.

Proof. Let $K$ be a hyperbolic 2-bridge knot in $S^{3}$ with exterior $M$. The result will follow from the previous proposition if we can show that $h\left(B_{\mathrm{M}}\right)<\frac{1}{2}$, a fact due to Tanguay [32].

Let $p: \widetilde{M} \rightarrow M$ be the (unique) 2 -fold cover and $\{\widetilde{\mu}, \widetilde{\lambda}\}$ the basis for $H_{1}(\partial \widetilde{M})$ corresponding to $\{\mu, \lambda\}$. Then $\widetilde{M}$ is hyperbolic and $\widetilde{M}(\widetilde{\mu})$ is 
the double branched cover of $S^{3}$ branched over $K$, which is known to be a lens space (see [7]). Thus $\widetilde{\mu}$ is a cyclic filling class of $\widetilde{M}$.

By the discussion proceeding Proposition 9.6 (cf. also [2, Section $3])$, the cover $p: \widetilde{M} \rightarrow M$ induces a regular map $p^{*}: X(M) \rightarrow X(\widetilde{M})$ and $E_{\mathrm{M}}=\overline{p^{*}\left(C_{\mathrm{M}}\right)}$ is a norm curve in $X(\widetilde{M})$ with norm $\|\cdot\|_{\widetilde{E}_{\mathrm{M}}}$. Moreover for every $\widetilde{\delta}$ in $H_{1}(\partial \widetilde{M})$ it can be shown that if $p_{\#}: H_{1}(\partial \widetilde{M}) \rightarrow H_{1}(\partial M)$ is the homomorphism induced by $p \mid \partial \widetilde{M}$, then

$$
\left\|p_{\#}(\widetilde{\delta})\right\|_{\mathrm{M}}=2\|\widetilde{\delta}\|_{\widetilde{E}_{\mathrm{M}}}
$$

Now $\widetilde{\mu}$ cannot be associated to an ideal point of $y \in \widetilde{E}_{\mathrm{M}}$ as otherwise $\mu$ would be associated to each of the ideal points of $\left(\widetilde{p}^{*}\right)^{-1}(y) \subset \widetilde{C}_{\mathrm{M}}$, which is impossible as it is not a boundary class. Thus $\|\widetilde{\mu}\|_{\widetilde{E}_{\mathrm{M}}} \leq\|\widetilde{\beta}\|_{\widetilde{E}_{\mathrm{M}}}$ for each nonzero $\widetilde{\beta} \in H_{1}(\partial \widetilde{M})$ (see Theorem 6.1 of [3]). Since even integer classes in $H_{1}(\partial M)$ lift to classes in $H_{1}(\partial \widetilde{M})$, it follows that

$$
\|\beta\|_{\mathrm{M}} \geq\|2 \mu\|_{\mathrm{M}}=2\|\mu\|_{\mathrm{M}}
$$

for every even integer slope $\beta$ on $\partial M$. We now show that this inequality holds strictly for all primitive classes $\beta \neq \pm \mu$, and so $h\left(B_{\mathrm{M}}\right)<\frac{1}{2}$.

Let $\beta$ be a boundary slope of $M$ associated to a vertex of $B_{\mathrm{M}}$, i.e., there is an ideal point $x \in \widetilde{C}_{\mathrm{M}}$ for which $f_{\beta}(x)$ is finite but $f_{\mu}(x)=$ $\infty$. According to Theorem 5.4 of [28], the components of the essential surfaces in $M$ associated to $x$ have one or two boundary components. This fact implies, by $\S 5$ of [8], that $f_{\beta}(x)=0$. If $I_{\mu}: X(M) \rightarrow \mathbb{C}$ denotes the evaluation map, then $f_{\mu}=I_{\mu}^{2}-4$ and $f_{\mu^{2}}=I_{\mu}^{2} f_{\mu}$. Hence $f_{\mu^{2}}(x)=\infty$. Therefore the norm of $\beta$ is strictly larger than that of $2 \mu$. It follows that the norm polygon $B_{\mathrm{M}}$ lies strictly below the horizontal half-integer line. Hence Proposition 18.1 implies that $K$ admits no nonmeridinal finite filling slope.

q.e.d.

\section{Appendix}

The goal of this appendix is to prove the following result.

Proposition. Let $\theta \in\{ \pm 1\}$. The polynomial $A_{\theta}(u, v)=u^{3}+(\theta-$ $\left.u-\theta u^{2}-\theta u^{4}-u^{5}+\theta u^{6}\right) v^{2}+u^{3} v^{4}$ is not the canonical polynomial of a hyperbolic knot exterior. 
Proof. Let $M$ be a compact, connected, orientable 3-manifold whose interior admits a complete hyperbolic structure of finite volume. Suppose further that the boundary of $M$ is a torus. In [8] it is described how work of C. Hodgson implies that the real 1-form

$$
\omega=\ln |u| d(\arg (v))-\ln |v| d(\arg (u))
$$

is exact on the smooth part of $D_{\mathrm{M}}$. In particular its integral over any closed, piecewise-smooth loop in $D_{\mathrm{M}}$ is zero. We'll show that this condition does not hold for the zero sets of the polynomials under consideration in the proposition. Arguments of this type were first used by D. Cooper and D. Long in $[9, \S 10]$.

First observe that we may assume that $\theta=1$. For if $\psi: A_{1}^{-1}(0) \rightarrow$ $A_{-1}^{-1}(0)$ is the isomorphism given by $(u, v) \mapsto(-u, v)$ and $C \subset A_{1}^{-1}(0)$ is a piecewise-smooth curve, then $\int_{C} \omega=\int_{\psi(C)} \omega$. Consider then

$$
A(u, v)=u^{3}+\left(1-u-u^{2}-u^{4}-u^{5}+u^{6}\right) v^{2}+u^{3} v^{4}
$$

and set

$$
D=A^{-1}(0) .
$$

The singular set of $D$ consists of the simultaneous solutions of the equations

$$
A=0, \quad \frac{\partial A}{\partial u}=0, \quad \frac{\partial A}{\partial v}=0
$$

and is readily calculated to be $\Sigma=\left\{(u, v) \mid u^{2}=v^{4}=1\right\}$. The projection induced map

$$
\pi: D \rightarrow \mathbb{C}, \quad \pi:(u, v) \mapsto u
$$

has degree 4 and is branched at the points of $\mathcal{B}=\frac{\partial A}{\partial v}^{-1}(0) \backslash \Sigma$. The automorphism $\phi:(u, v) \mapsto(u,-v)$ of $D$ satisfies $\pi \circ \phi=\pi$, so the branching at a point $(u, v) \in D$ is necessarily of order 2 if $v \neq 0$. Set $u_{0}=\frac{1}{4}(1-\sqrt{17})-\frac{i}{2} \sqrt{2 \sqrt{17}-2}$ and $u_{1}=i$. It is easy to verify that

$$
\left(u_{0}, i\right),\left(u_{0},-i\right),\left(u_{1}, i\right),\left(u_{1},-i\right) \in \mathcal{B} \text {. }
$$

The path $u(t)=(1-t) u_{0}+t u_{1}$ in the $u$-plane lifts to four smooth paths $\sigma_{j}(t)=\left(u(t), v_{j}(t)\right)(j=1,2,3,4)$ in $D$ which we can determine as follows. Any lift $v(t)$ of $u(t)$ satisfies the identity

$$
1+g(t) v(t)^{2}+v(t)^{4}
$$


where

$$
g(t)=u^{-3}(t)-u^{-2}(t)-u^{-1}(t)-u(t)-u^{2}(t)+u^{3}(t) .
$$

Solving for $v(t)$ shows that

$$
v(t)= \pm \sqrt{\frac{-g(t) \pm \sqrt{g(t)^{2}-4}}{2}} .
$$

The reader may verify that $g(t)= \pm 2$ if and only if $t \in\{0,1\}$, and so there are precisely two smooth functions $r_{1}(t)$ and $r_{2}(t)$ whose square is $g(t)^{2}-4$. They are unambiguously determined by requiring that $\operatorname{Re}\left(r_{1}(t)\right) \geq 0$ and $\operatorname{Re}\left(r_{2}(t)\right) \leq 0$ when $t$ is close to zero. Evidently $r_{2}=-r_{1}$. Next note that since $-g(t)+r_{1}(t) \neq 0$, there are exactly two smooth functions $v_{1}, v_{3}$ satisfying $v_{1}^{2}=v_{3}^{2}=\frac{1}{2}\left(-g+r_{1}\right)$. They are determined by requiring that $v_{1}(0)=i$ while $v_{3}(0)=-i$. Similarly there are exactly two smooth functions $v_{2}, v_{4}$ satisyfying $v_{2}^{2}=v_{4}^{2}=\frac{1}{2}\left(-g+r_{2}\right)$ and they are determined by requiring that $v_{2}(0)=i$ while $v_{4}(0)=-i$. Evidently $v_{3}=-v_{1}$ and $v_{4}=-v_{2}$. We take

$$
\sigma_{j}(t)=\left(u(t), v_{j}(t)\right)
$$

It follows from our choices that

$v_{1}^{2} v_{2}^{2}=\frac{1}{4}\left(-g+r_{1}\right)\left(-g+r_{2}\right)-1=\frac{1}{4}\left(-g+r_{1}\right)\left(-g-r_{1}\right)=\frac{1}{4}\left(g^{2}-r_{1}^{2}\right)=1$.

and hence for each $t \in[0,1]$, we have $v_{1}(t) v_{2}(t)=v_{1}(0) v_{2}(0)=-1$. In particular since $v_{1}(1)=\epsilon i$ for some $\epsilon \in\{ \pm 1\}$, we must have $v_{2}(1)=\epsilon i$ as well. It follows that $C=\sigma_{1} * \sigma_{2}^{-1}$ is a closed, piecewise-smooth curve in $D$. Now

$$
\int_{C} \omega=\int_{\sigma_{1}} \omega-\int_{\sigma_{2}} \omega
$$

while from the relation $v_{1} v_{2}=-1$ and the form of the integrand we see that

$$
\int_{\sigma_{1}} \omega=-\int_{\sigma_{2}} \omega
$$

Hence

$$
\int_{C} \omega=2 \int_{\sigma_{1}} \omega
$$


To compute the latter we proceed as follows. One can verify that if $w(t)$ is a smooth path in the complex plane, then $\frac{d}{d t} \arg (w(t))=\operatorname{Im}\left(\frac{w^{\prime}(t)}{w(t)}\right)$. It follows that

$$
2 \int_{\sigma_{1}} \omega=-\int_{0}^{1}\left\{\ln \left(|u(t)|^{2}\right) \operatorname{Im}\left(\frac{g^{\prime}(t)}{2 r_{1}(t)}\right)-\ln \left(\left|v_{1}(t)\right|^{2}\right) \operatorname{Im}\left(\frac{u^{\prime}(t)}{u(t)}\right)\right\} d t
$$

Let $f(t)$ be the integrand of this integral. Its graph is depicted in Figure 13.

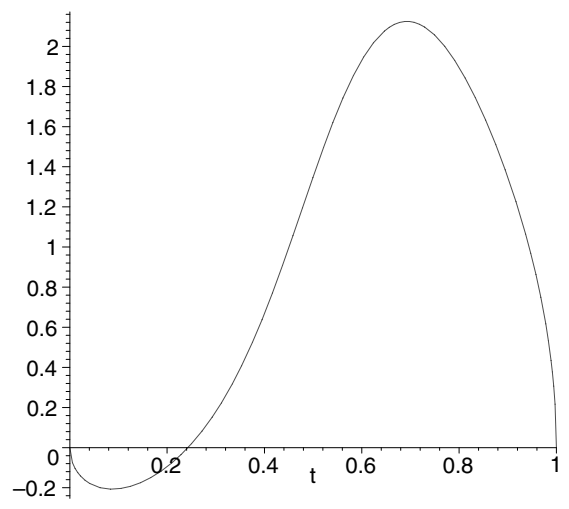

Figure 13: The graph of $f(t)$.

Now with $n=10,000$ we have

$$
\begin{aligned}
& \left|\int_{0}^{1} f(t) d t-\sum_{j=1}^{n} \frac{f\left(\frac{j}{n}\right)}{n}\right| \\
& \leq\left|\int_{0}^{\frac{1}{n}} f(t) d t\right|+\left|\frac{f\left(\frac{1}{n}\right)}{n}\right|+\left|\int_{\frac{1}{n}}^{\frac{n-1}{n}} f(t) d t-\sum_{j=2}^{n-1} \frac{f\left(\frac{j}{n}\right)}{n}\right| \\
& \quad+\left|\int_{\frac{n-1}{n}}^{1} f(t) d t\right|+\left|\frac{f(1)}{n}\right| \\
& \leq \frac{4 K}{n}+\frac{(n-2) L}{n^{2}} \\
& <\frac{4 K+L}{10,000}
\end{aligned}
$$


where

$$
\begin{aligned}
& K=\sup \left\{|f(t)| \mid t \in\left[0, \frac{1}{n}\right] \cup\left[\frac{n-1}{n}, 1\right]\right\}, \\
& L=\sup \left\{\left|f^{\prime}(t)\right| \mid t \in\left[\frac{1}{n}, \frac{n-1}{n}\right]\right\} .
\end{aligned}
$$

Crude estimates show that $K<2$ while $L<1,700$. Thus

$$
\left|\int_{0}^{1} f(t) d t-\sum_{j=1}^{n} \frac{f\left(\frac{j}{n}\right)}{n}\right|<\frac{1,708}{10,000}<0.2
$$

and so

$$
\left|\int_{0}^{1} f(t) d t\right| \geq\left|\sum_{j=1}^{n} \frac{f\left(\frac{j}{n}\right)}{n}\right|-0.2 .
$$

Computer assisted calculation shows $\left|\sum_{j=1}^{n} \frac{f\left(\frac{j}{n}\right)}{n}\right|>0.94$ so that we conclude

$$
\int_{C} \omega=2 \int_{\sigma_{1}} \omega=-\int_{0}^{1} f(t) d t \neq 0
$$

This completes the proof of the proposition.

q.e.d.

\section{References}

[1] S. Bleiler \& C. Hodgson, Spherical space forms and Dehn fillings, Topology 35 (1996) 809-833.

[2] S. Boyer \& X. Zhang, Finite Dehn surgery on knots, J. Amer. Math. Soc. 9 (1996) 1005-1050.

[3] S. Boyer \& X. Zhang, Culler-Shalen seminorms and Dehn fillings, Annals of Math., to appear.

[4] S. Boyer \& X. Zhang, A note on finite Dehn filling, Canadian Math. Bulletin, to appear.

[5] S. Boyer \& X. Zhang, On simple points of character varieties of 3-manifolds, preprint.

[6] S. Boyer, T. Mattman, \& X. Zhang, The fundamental polygons of twist knots and the $(-2,3,7)$ pretzel knot, Knots' 96, World Scientific Publishing Co. Pte. Ltd. (1997) 159-172. 
[7] G. Burde \& H. Zieschang, Knots, de Gruyter, 1985.

[8] D. Cooper, M. Culler, H. Gillet, D.D. Long, \& P. Shalen, Plane curves associated to character varieties of 3-manifolds, Invent. Math. 118 (1994) 47-84.

[9] D. Cooper \& D.D. Long, Remarks on the A-polynomial of a knot, J. of Knot Theory and Its Ramifications 5 (1996) 609-628.

[10] D. Cooper \& D.D. Long, The A-polynomial has ones in the corners, Bull. London Math. Soc. 29 (1997) 231-238.

[11] M. Culler, C.M. Gordon, J. Luecke, \& P. Shalen, Dehn surgery on knots, Ann. of Math. 125 (1987) 237-300.

[12] M. Culler \& P. Shalen, Varieties of group representations and splittings of 3manifolds, Ann. of Math. 117 (1983) 109-146.

[13] Bounded, separating surfaces in knot manifolds, Invent. Math. 75 (1984) 537545.

[14] C. Delman, Essential laminations and Dehn surgery on 2-bridge knots, Top. Appl. 63 (1995) 201-221.

[15] N. Dunfield, Cyclic surgery, degrees of maps of character curves, and volume rigidity for hyperbolic manifolds, preprint, 1998.

[16] C. Gordon, Dehn surgery on knots, Proc. Int. Congress of Mathematicians, Kyoto (1990) 631-642.

[17] C. Gordon, Dehn filling: a survey, Proc. Mini Semester in Knot Theory, Warsaw 1995, to appear.

[18] W. Hatcher and W. Thurston, Incompressible surfaces in 2-bridge knots complements, Inv. Math. 79 (1985) 225-246.

[19] J. Hempel, 3-manifolds, Ann. of Math Studies 86 (1976).

[20] W. Jaco, Lectures on three-manifold topology, CBMS Regional Conf. Ser. Math. 43 (1980).

[21] R. Kirby, Problems in low-dimensional topology, Geometric Topology, Volume 2, editor W. Kazez, AMS/IP Studies in Advanced Mathematics, 1996.

[22] S. Lang, Introduction to algebraic geometry, Interscience Tracts in Pure and Applied Mathematics 51958.

[23] S. Lang, Algebra, Addison-Wesley Series in Mathematics, 1965.

[24] J. Luecke, Dehn surgery on knots in $S^{3}$, Proceedings of Int. Congress of Mathematicians, Zurich (1995) 585-594.

[25] J. Milnor, Groups which act on $S^{n}$ without fixed points, Amer. J. Math. 79 (1957) 623-631. 
[26] J. Morgan \& P. Shalen, Degenerations of hyperbolic structures, III: Actions of 3-manifold groups on trees and Thurston's compactness theorem, Ann. of Math. 127 (1988) 457-519.

[27] L. Moser, Elementary surgery along a torus knot, Pacific J. Math. 38 (1971) $737-745$.

[28] T. Ohtsuki, Ideal points and incompressible surfaces in two-bridge knot complements, J. Math. Soc. Japan 46 (1994) 51-87.

[29] D. Rolfsen, Knots and links, 2nd edition, Publish or Perish, 1990.

[30] I. Shafarevich, Basic algebraic geometry, Die Grundlehren der mathematischen Wissenschaften, Band 213, Springer-Verlag, New York, 1974.

[31] P. Shanahan, Cyclic Dehn surgery and the A-polynomial, preprint.

[32] D. Tanguay, Chirurgie finie et noeuds rationnels, Ph.D. thesis, UQAM, 1995.

[33] W. Thurston, Three dimensional manifolds, Kleinian groups and hyperbolic geometry, Bull. Amer. Math. Soc. 6 (1982) 357-381.

[34] W. Thurston, The geometry and topology of three manifolds, Lecture Notes, Princeton, 1979.

[35] J. Weeks, Ph. D. thesis, Princeton University, 1985.

[36] J.A. Wolf, Spaces of constant curvature, McGraw-Hill Series in Higher Mathematics, 1967.

Département de mathématiques, UQAM
Montreal H3C 3P8, CANAdA
Department OF MAThematics
SUNY Buffalo, Buffalo, NY 14214-3093 